\author{
UNIVERSIDADE DE SÃO PAULO \\ INSTITUTO DE FÍSICA DE SÃO CARLOS
}

\title{
MODELOS DE VÉRTICES EXATAMENTE INTEGRÁVEIS
}

\author{
Anderson Augusto Ferreira
}

Dissertação apresentada ao

Instituto de Fúsica de São Carlos, Universidade de São Paulo, para a obtenção do título de Mestre em Ciências: Física Básica

Orientador: Prof. Dr. Francisco Castilho Alcaraz

São Carlos, Março 2005 
Aos meus familiares e aos meus amigos. 
Este trabalho teve apoio financeiro da FAPESP . 


\section{A g r a d e c i m e n t o s}

- Ao professor Francisco Castilho Alcaraz, pelo grande esforço que tem feito no meu processo de formação científica.

- A minha mãe, ao meu irmão, e ao meu amigo Justino, que me deram muita força nos momentos difíceis de minha vida.

- Ao meu Amigo Matheus, pelas longas conversas sobre Física e Matemática. 


\section{R e s u m o}

Nesta dissertação, mostramos as primeiras aplicações do recém criado Ansatz do Produto Matricial [8] na solução exata das matrizes de transferência associadas a modelos de vértices. A integrabilidade dos modelos é obtida diagonalizando-se a matriz de transferência diagonal-para-diagonal. Foram estudados duas classes de modelos.

$\mathrm{Na}$ primeira delas introduzimos novos modelos de vértices, que denominamos de modelos de 5 vértices interagentes. Nestes modelos os vértices além das interações usuais de vizinhos próximos, dadas pela regra do gelo, possuem também interações de natureza repulsiva ao longo da diagonal. O famoso modelo de 6 vértices é obtido num limite particular deste novo modelo.

O espectro da matriz de transferência, analogamente ao que acontece no ansatz de Bethe tradicional é dado em termos da solução de equações não lineares. Um estudo analítico e numérico destas equações foi feito para o modelo de 6 vértices que está contido nesta primeira classe de modelos. Tais resultados, juntamente com as idéias de invariância conforme, nos permitiram estudar o modelo em seu regime crítico. A segunda classe de modelos que estudamos foram os modelos de 10 vértices que satisfazem às regras do gelo. Obtivemos todos os possíveis modelos exatamente integráveis desta classe, reobtendo resultados da literatura bem como novos resultados. 


\begin{abstract}
In this dissertation we present the first application of a recent introduces Matrix Product Ansatz [8], in the exact solution of the transfer matrices associated to vertex models. The exact integrability is obtained thruong the diagonalization of the diagonal-to-diagonal transfer matrix. We studied two classes of models. In the first one we introduce new vertex models, thet we call as interacting 5 vertex models. On these models beyond the nearest-neighbour interactions amongthe vértices, imposed by the ice rule, they also have repulsive interactions along the diagonal. The famous 6 vertex model is just a special casethis class of models.

The eigenspectrum of this transfer matrix, analagously as in the the traditional Bethe ansatz, is obtained in terms of the roots of non linear equation. An analitical e numerical study of these equations we done in this case of the 6 vertex model wich is an special case of the models considered on the first class. These results together with the machinary comming from conformal invariance allow us the study the model on its critical region.

The second class of models we considered were the 10 vertex models that satisfy ice rules we obtained all the possible exact integrabel models on this class, rederiving earlier results on the literature as were producing new ones.
\end{abstract}




\section{Conteúdo}

2 Modelos de vértices com condições de contorno toroidais especiais 9

2.1 Introdução . . . . . . . . . . . . . . . . . . . . . . . . . 9

2.2 Localização das flechas na rede e a matriz de transferência diagonalpara-diagonal . . . . . . . . . . . . . . . . . . . 12

3 Modelos de 5 vértices interagentes

3.1 Modelo de 5 vértices com domínio de interação $s$ fixo $\ldots$. . . . . . 16

3.2 Modelo de 5 vértices com domínio de interação $s$

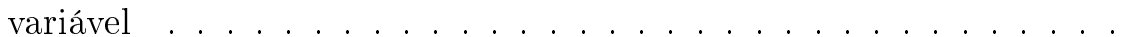

3.3 Diagonalização da matriz de transferência diagonal-para-diagonal do modelo de 5 vértices interagentes de domínio de interação $s$ variável . . . . . . . . . . . . . . . . . 19

3.4 Estrutura dos autovalores da matriz de transferência do modelo de seis vértices. . . . . . . . . . . . . . . . . . 36

3.4.1 Soluções Numéricas para cadeias finitas . . . . . . . . . . . . 36

3.5 Limite Termodinâmico . . . . . . . . . . . . . . . . . . . . 44

3.5.1 Introdução . . . . . . . . . . . . . . . . . . . . . 44

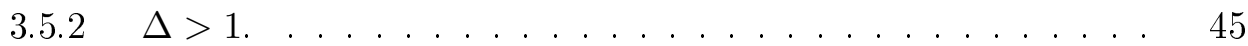


$3.5 .3-1<\Delta<1 \ldots \ldots \ldots \ldots \ldots \ldots \ldots$

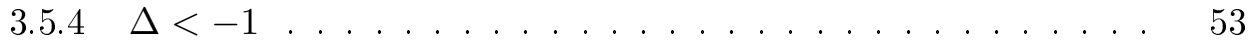

3.6 Conteúdo operatorial do modelo de 6 vértices a partir da matriz de transferência diagonal-para diagonal . . . . . . . . . . . . 55

3.6.1 Velocidade do som em $\Delta=0 \ldots \ldots \ldots \ldots$

3.6.2 Estados "excitados" . . . . . . . . . . . . . . . . . 58

4 Modelos de 10 vértices exatamente integrável na rede diagonal 62

4.1 Descrição do modelo . . . . . . . . . . . . . . . . . . . . . 62

4.2 Diagonalização da matriz de transferência diagonal-para-diagonal associada ao modelo de 10 vértices $\ldots \ldots \ldots$. . . . . . . . 63

4.3 Análise das soluções . . . . . . . . . . . . . . . . . . . . . 83

5 Conclusão $\quad 88$

$\begin{array}{llr}6 & \text { Bibliografia } & 91\end{array}$ 


\section{Capítulo 1}

\section{Introdução}

No início do século 20, Niels Bohr e a física holandesa J.H. Leeuwen mostraram que em qualquer temperatura finita, e para quaisquer campos elétricos e magnéticos finitos, a magnetização resultante de uma coleção de elétrons em equilíbrio térmico é identicamente igual a zero. Tal demostração contrariava a experiência, pois desde a era grega, o homem já tinha conhecimento da existência de materiais magnéticos. Evidentemente com essa demonstração, a teoria clássica mostrava-se incapaz de explicar o comportamento magnético dos materias. Apenas com o advento da Mecânica Quântica foi possível entender que a principal causa do magnetismo era devido à interaçâo coulombiana e a simetria imposta pelos spins dos elétrons.

Ising em 1925, propôs um modelo semi-clássico de spins em uma dimensão, com o objetivo de tentar explicar a transição de fase do estado paramagneto para o estado ferromagneto, e consequentemente estruturar a teoria dos materias magnéticos. O seu modelo era caracterizado pela Hamiltoniana

$$
E(X)=-J \sum_{i=1}^{L} s_{i} s_{i+1}-h \sum_{i=1}^{L} s_{i}
$$

onde $J, h$ são constantes que dão conta das interações dos spins entre si e com um campo magnético externo, respectivamente. O conjunto $X=\left\{s_{1}, \ldots, s_{L}\right\}$ caracteriza 
as possíveis configurações da rede. As variáveis $s_{k}, k \in(1, \ldots, L)$ assumem dois possíveis valores $(1,-1)$, correspondendo às duas possíveis orientações dos spins em cada sítio $i(i=1, \ldots, L)$ da rede.

Baseando-se neste modelo, Ising calculou exatamente a função de partição, e consequentemente obteve as grandezas termodinâmicas. A conclusão de seus cálculos indicavam para campo magnético externo nulo, a existência de uma transição de fase do estado paramagneto para o estado ferromagneto somente em $T=0$, contradizendo os resultados experimentais que indicavam transições de fase em $T \neq 0$. Em virtude deste fato, Heisenberg propôs em 1928 um modelo quântico de spin. Neste modelo, que passou a ser chamado de modelo de Heisenberg, as variáveis de spin são trocadas por matrizes de Pauli, isto é,

$$
H=-J \sum_{<r, r^{\prime}>}\left(\overrightarrow{\sigma_{r}} \vec{\sigma}_{r^{\prime}}-1\right)-h \sum_{<r>} \sigma_{r}^{z}
$$

onde $\vec{\sigma}_{r}=\left(\sigma_{r}^{x}, \sigma_{r}^{y}, \sigma_{r}^{z}\right)$ são as matrizes de Pauli de spin $\frac{1}{2}$, localizados nos sítios $\vec{r}$ da rede, e $\left\langle r, r^{\prime}>\right.$ são as coordenadas dos vizinhos próximos na rede.

A versão unidimensional deste modelo foi resolvida em 1931 por Hans Bethe. Em 1966 Yang e Yang [1] resolveram a versão anisotrópica do modelo de Heisenberg unidimensional. No ano seguinte Lieb [2] resolveu o modelo de seis vértices que discutiremos abaixo. Ambas as soluções foram obtidas utilizando-se as idéias de Bethe. Iniciava-se então, a era dos sistemas exatamente integráveis.

A idéia do ansatz de Bethe ${ }^{1}$ consiste em escrever as autofunções (estado de Bethe) do operador (Hamiltoniano ou matriz de transferência), como uma combinação linear dos vetores de base sendo as amplitudes combinações lineares de ondas planas.

A aplicação do operador a ser diagonalizado nos estados de Bethe fornecem relações que no caso do modelo ser exatamente integrável, são simultâneamente satisfeitas.

\footnotetext{
${ }^{1}$ Esta versão original do ansatz de Bethe é comumente denominada por ansatz de Bethe de coordenadas
} 
Tais relações são expressas por um conjunto de equações não lineares denominadas por equações do ansatz de Bethe. O espectro do operador é obtido mediante a resolução destas equações.

O modelo de seis vértices foi proposto em 1932 por L. Pauling para explicar a entropia residual (entropia por sítio) não nula no zero absoluto de algumas substâncias, como por exemplo o monóxido de carbono, hidrogênio e o gelo. Pauling, numa versão simplificada adimitiu que o cristal de gelo fosse formado por um arranjo cristalino em que os oxigênios ocupassem os vértices de uma rede quadrada e os hidrogênios as ligações da mesma (pontes de hidrogênio). Estas ligações deveriam satisfazer à seguinte regra.

Regra do gelo: Ao redor de um átomo de oxigênio existem outros 4 átomos de oxigênio numa distância de 2.76 Angstrons. Os oxigênios são ligados por pontes de hidrogênio, que correspondem a um poço duplo de potencial com mínimo às distâncias de 0.95 Angstrons e de 1.81 Angstrons. Para a neutralidade local de carga ao redor de cada oxigênio, dois dos hidrogênios devem estar à distâncias menor e dois à distâncias maior.

Segundo esta regra temos 6 possibilidades (vértices) de arranjar os hidrogênios ao redor de um átomo de oxigênio. Mostramos na primeira fileira da figura 1 uma possível representação da regra do gelo. Nesta figura as setas entrando representam os hidrogênios próximos e as setas saindo representam os hidrogênios mais distantes de um determinado oxigênio que situa-se no vértice.

Quase trinta anos mais tarde, Lieb diagonalizou de forma exata a matriz de transferência (linha-para-linha) associada ao modelo de 6 vértices, para o caso especial em que todas as energias eram iguais $\left(e_{1}=e_{2}=\ldots=e_{6}=e\right)$, entretanto no modelo mais geral de seis vértices [3] podemos atribuir energias distintas para cada configuração local de oxigênios que seguem a regra do gelo, como denotado na figura 1. 


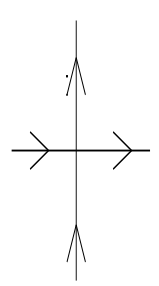

$e_{0}$

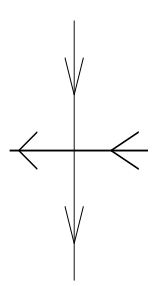

$e_{1}$

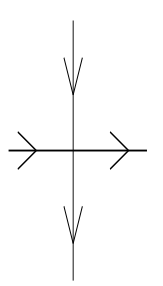

$e_{2}$
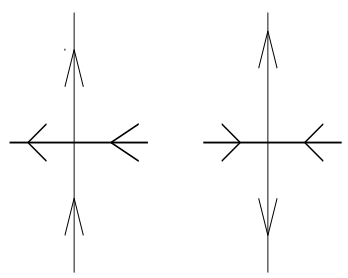

$e_{4}$

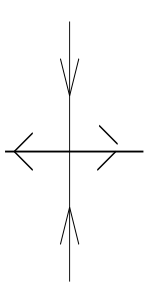

$e_{5}$

Figura 1.1: Energias dos vértices.

Modelos com valores especiais de energias tem recebido diferentes denominações na literatura [3]. O modelo conhecido como KDP, que descreve o comportamento ferroelétrico em baixas temperaturas do di-hidrogênio fosfato de potássio é obtido quando $e_{1}=e_{2}=0, e_{3}=e_{4}=e_{5}=e_{6}>0$. Um outro modelo conhecido por modelo $F$ caracteriza o comportamento antiferroelétrico. As energias deste modelo são $e_{1}=e_{2}=e_{3}=e_{4}>0, e_{5}=e_{6}=0$.

Por outro lado, a representação de processos estocásticos interagentes em termos de cadeias quânticas de spins tem produzido uma frutífera conexão entre a mecânica estatística do equilíbrio e a do não equilíbrio. O mapeamento entre estas duas áreas deve-se à similaridade entre a equação mestra que descreve as flutuações temporais de não equilíbrio de um dado processo estocástico e as flutuações quânticas no equilíbrio de cadeias quânticas de spin.

A conexão matemática entre o equílibrio e o não equilíbrio revela que algumas cadeias quânticas que descreve certos processos estocásticos interagentes são exatamente integráveis via ansatz de Bethe.

Mais recentemente, ao longo da década de noventa e início desta década [30]-[31], surgiram trabalhos onde se mostravam que a distribuições estacionárias de probabilidades de alguns modelos estocásticos, poderiam ser expressos em termos de um 
ansatz de produto de matrizes (Matrix Product Ansatz). De acordo com este ansatz as componentes do autovetor do estado fundamental são dadas em termos de um produto de matrizes. Estas componentes, a menos de uma constante de normalização, são fixas pelas relações de comutação das matrizes, definindo deste modo o ansatz de produto de matrizes. Estes modelos são em geral não exatamente integráveis, e o ansatz do produto de matrizes somente fornece o autovetor do estado fundamental do Hamiltoniano quântico associado ao modelo estocástico, em contraposição ao ansatz de Bethe acima mencionado, em que um número infinito de autofunções são obtidas. Recentemente surgiu um novo ansatz de produto de matrizes na área de modelos estocásticos interagentes, ansatz este denominado por ansatz do produto matricial dinâmico (Dynamic Matrix Product Ansatz [4]). De acordo com este ansatz, a distribuição de probabilidade do modelo é dada por um produto de matrizes não apenas para o estado estacionário, mas sim para um tempo arbitrário. O ansatz do produto matricial dinãmico foi aplicado originalmente em problemas de difusão assimétrica de partículas na rede [4], [5]. Recentemente [6], [7] mostraram que o ansatz pode ser também aplicado em problemas de difusão assimétrica contendo duas classes de partículas. Motivados por este ansatz Alcaraz e Lazo [8] perceberam que o sucesso do ansatz matricial nos processos estocásticos acima mencionado [4]-[7] só poderiam ser explicados pelo fato dos Hamiltonianos quânticos associados serem exatamente integráveis. Isto possibilitou [8] a formulação de um novo ansatz do produto matricial, que pode ser aplicado, em princípio, para cadeias quânticas exatamente integráveis, estejam tais cadeias ligadas ou não a processos estocásticos. Em princípio tal formulação teria o mesmo grau de abrangência que o famoso ansatz de Bethe.

A idéia básica deste ansatz consiste em escrever as autofunções do operador a ser diagonalizado como uma combinação linear dos vetores de base, cujas amplitudes são escritas como o traço de um produto de matrizes.

Quando o modelo é exatamente integrável, as matrizes que definem o ansatz possuem relação de comutação bem definidas. As equações que fixam o espectro do modelo 
(análogas às equações do ansatz de Bethe mencionadas anteriormente) são obtidas através da invariância cíclica do traço de produtos matriciais, juntamente com as relações de comutação entre as matrizes definidas no ansatz matricial.

Paralelamente ao desenvolvimento dos sistemas exatamente solúveis, desenvolvia-se a teoria dos fenômenos críticos, cujo ponto culminante foi dado em 1974 com as idéias da teoria do Grupo de Renormalização por Wilson [9] que proporciou um maior entendimento das classes de universalidade de comportamento crítico.

Os ingredientes básicos que caracterizavam sistemas físicos pertencentes às diferentes classes de universalidades, seriam rotulados pela sua dimensionalidade e suas simetrias. As singularidades das funções termodinâmicas estariam governadas pelos pontos fixos do grupo de renormalização. Em 1984 Belavin, Polyakov e Zamolodchikov (BPZ) [10] perceberam que se os sistemas críticos fossem, além de invariantes por dilatação (como no grupo de renormalização), também invariantes por transformações conforme, grandes consequências surgiriam em duas dimensões, onde o grupo das transformações conformes é de dimensão infinita. O comportamento dos fenômenos críticos agora estariam indexados por um número $c$, chamado de anomalia central ou carga central da algebra conforme.

Em seguida, Friedan, Qiu e Shenker [11] mostraram que se além de invariantes conformes os modelos também forem unitários (que é o caso dos modelos estatísticos que são associados à matrizes de transferência unitárias) e com a carga central menor que um $(c \leq 1)$, as possíveis classes de universalidades são quantizadas pela série

$$
c=1-\frac{6}{m(m+1)}, \quad m=2,3, \ldots,
$$

série esta denominada minimal. Para tal série as dimensões escalares $\Delta_{p, q}$ (que estão associadas aos expoentes críticos) é dada pela fórmula de Kac [12]

$$
\Delta_{p, q}=\frac{[p(m+1)-q m]^{2}-1}{4 m(m+1)}, 1 \leq p \leq q \leq m
$$


A tabela dos possíveis pesos conformes para um dado valor da anomalia conforme é também chamada por tabela de Kac.

No caso em que a anomalia conforme é maior que um $(c \geq 1)$, a unitariedade e a invariância conforme não são suficientes para quantizar os possíveis valores de $c$, a não ser que simetrias adicionais sejam impostas.

A fase marcante da aplicação da invariância conforme em fenômenos críticos à duas dimensões surgiu com os trabalhos de Blöte et al. [13], Affleck [14] e Cardy [15],[16]. Eles mostraram uma maneira sistemática de se estimar a anomalia conforme $c$ e as dimensões anômalas dos operadores (que estão associadas com os expoentes críticos) a partir do comportamento assintótico do espectro dos modelos em sua formulação Euclidiana (matriz de transferência) ou Hamiltoniana em geometrias finitas.

Blöte et al. [13] e Affleck [14] mostraram, independentemente, que se escrevermos a matriz de transfêrencia $T$ como $T=e^{-H}$, então a anomalia conforme $c$ pode ser determinada a partir do comportamento assintótico do estado fundamental de $H$ quando definido numa tira de tamanho $L$, e com bordas periódicas, da seguinte maneira

$$
\frac{E_{0}(L)}{L}=e_{\infty}-\frac{\pi c v_{s}}{6 L^{2}}+o\left(L^{-2}\right)
$$

sendo $e_{\infty}$ a energia por sítio do estado fundamental da rede infinita e $v_{s}$ (velocidade do som) o coeficiente linear na relação de dispersão energia-momento da cadeia. Já as dimensões dos operadores que governam as flutuações críticas são calculadas a partir do comportamento assintótico $(L \rightarrow \infty)$ dos estados excitados de $H$. Para cada operador primário $O_{\Delta, \bar{\Delta}}$ com dimensão $x^{\Delta, \bar{\Delta}}=\Delta+\bar{\Delta}$ e spin $s^{\Delta, \bar{\Delta}}=\Delta-\bar{\Delta}$, existe uma torre infinita de estados de $H$, cujas energias $E_{m, m^{\prime}}^{\Delta, \bar{\Delta}}$ e momento $P_{m, m^{\prime}}^{\Delta, \bar{\Delta}}$, na cadeia periódica, são dados por 


$$
\begin{aligned}
& E_{m, m^{\prime}}^{\Delta, \bar{\Delta}}(L)=E_{0}+\frac{2 \pi v_{s}}{L}\left(x^{\Delta, \bar{\Delta}}+m+m^{\prime}\right)+o\left(L^{-1}\right), \\
& P_{m, m^{\prime}}^{\Delta, \bar{\Delta}}=\frac{2 \pi}{L}\left(s^{\Delta, \bar{\Delta}}+m-m^{\prime}\right) .
\end{aligned}
$$

A disposição dos capítulos desta dissertação foi elaborada de modo a permitir que o leitor tivesse uma visão sequencial do que foi estudado e desenvolvido durante este período de mestrado. No capítulo 2 apresentamos de forma didática a cosntrução da matriz de transferência diagonal-para-diagonal associada aos modelos de vértices estudados nesta dissertação.

No capítulo 3 introduzimos novos modelos de vértices que denominamos por modelos de 5 vértices interagentes, sendo que, o famoso modelo de 6 vértices pode ser obtido como um caso particular deste novo modelo. Neste mesmo capítulo utilizamos o ansatz do produto matricial, para obter a solução exata destes novos modelos associados à matriz de transferência diagonal-para-diagonal. Em seguida, faremos um estudo numérico das equações do ansatz de Bethe do modelo de 6 vértices associado à matriz de transferência diagonal-para-diagonal, que serviu como ponto de partida para extrairmos o limite termodinâmico deste modelo. Para concluir este capítulo, extraímos o conteúdo operatorial do modelo de 6 vértices, em sua região crítica, que compreende o intervalo $-1<\Delta<1$, onde $\Delta$ é a anisotropia que caracteriza o modelo de 6 vértices.

No capítulo 4 apresentamos e obtemos a solução exata dos modelos de 10 vértices associados à matriz de transferência diagonal-para-diagonal utilizando o ansatz do produto matricial.

No capítulo 5, apresentamos nossas conclusões e possíveis extensões para o presente trabalho. 


\section{Capítulo 2}

\section{Modelos de vértices com condições de contorno toroidais especiais}

\subsection{Introdução}

Mostraremos aqui um método alternativo de resolver o problema de vértices na rede quadrada. Este método foi proposto por Bariev [17]. Ao invés de considerarmos a rede com condição de contorno periódica ao longo da horizontal consideraremos uma rede com condições de contorno toroidais especiais.

Para explicarmos estas condições toroidais especiais precisaremos nomear de uma forma especial os sítios numa rede quadrada formada por $M$ linhas e $N$ colunas. Uma rede com esta geometria possui $N \times M$ sítios. Um sítio é formado pela intersecção de uma linha com uma coluna. Chamaremos as linhas por $l(l=1, \ldots, M)$ e as colunas por $c(c=1, \ldots, N)$. Vamos agora nomear da esquerda para a direita da folha de papel, os sítios de uma determinada linha $l$ da seguinte forma:

$$
\left\{s^{(l)}\right\} \equiv\left(1^{(l)}, 2^{(l)}, \ldots, N^{(l)}\right) .
$$

Seguindo esta convenção mostramos na figura 2.1 a numeração dos sítios numa rede 
formada por $M$ linhas e $N$ colunas.

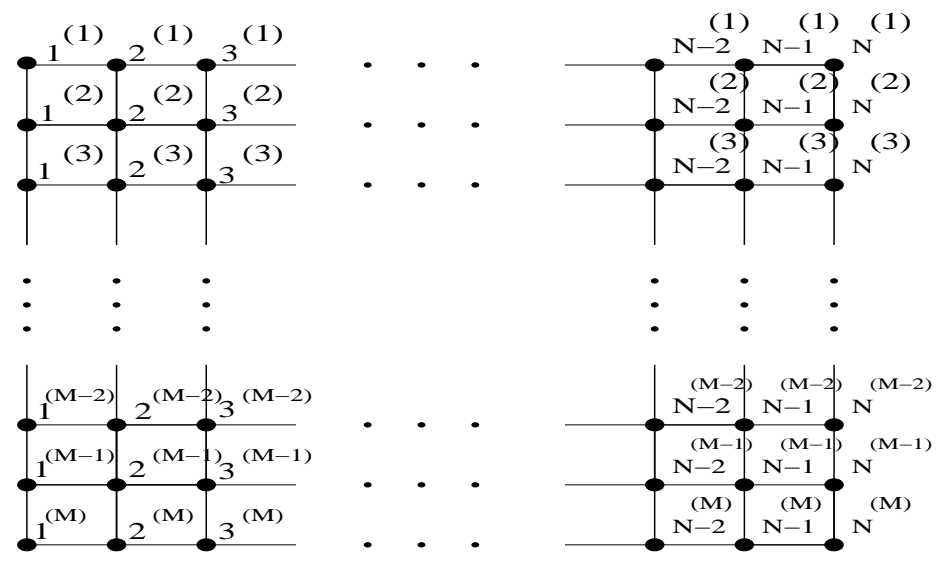

Figura 2.1: Localização dos sítios na rede quadrada.

Usando esta notação, as condições toroidais especiais de contorno são definidas de forma que um determinado sítio $s^{(l)}(s=1,2, \ldots, N)$ da linha $l(l=1,2, \ldots, M)$ será equivalente ao sítio $s^{(M+l)}$, e ao sítio $(M+s-1)^{(M+l-1)}$, ou seja,

$$
s^{(l)} \equiv s^{(M+l)},
$$

$$
s^{(l)} \equiv(M+s-1)^{(M+l-1)} .
$$

É interessante ressaltar que a denominação "especial" na condição de contorno que estamos usando deve-se à (2.3). Para calcular a matriz de transferência com as condições de contorno acima definidas será mais fácil representarmos a rede quadrada numa forma "diagonal" ${ }^{1}$ Na figura $3 b$ mostramos tal representação para a rede $N \times M=4 \times 5$.

Note que segundo as condições de contorno (2.2) temos para a rede da figura $2.2 a$ a equivalência dos seguintes sítios

\footnotetext{
${ }^{1}$ por simplicidade chamaremos esta rede deformada como rede diagonal.
} 


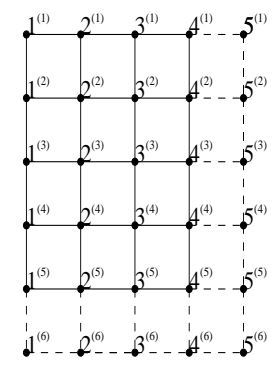

a)

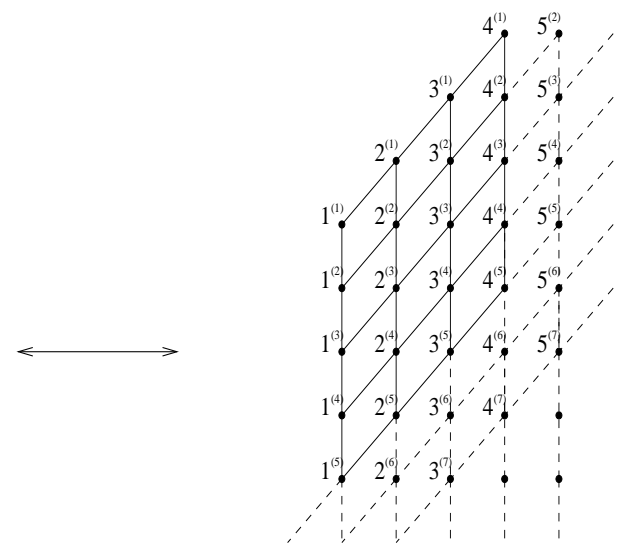

b)

Figura 2.2: Transformação da rede quadrada para diagonal.

$$
1^{(1)} \equiv 5^{(5)}, 1^{(2)} \equiv 5^{(6)}, 1^{(3)} \equiv 5^{(7)}, 1^{(4)} \equiv 5^{(8)}, 1^{(5)} \equiv 5^{(9)},
$$

enquanto que a condição de contorno (2.3) nos dá

$$
1^{(1)} \equiv 1^{(6)}, 2^{(2)} \equiv 2^{(7)}, 3^{(3)} \equiv 3^{(8)}, 4^{(4)} \equiv 4^{(9)} \text {. }
$$

Fazendo um corte na rede da figura $2 b$ de tal forma que o contorno seja dado pelos conjuntos de sítios em (2.4) e (2.5) obtemos a rede mostrada na figura 2.3.

Percebemos então que a rede escrita na geometria da figura 2.3 tem a virtude de possuir as condições de contorno do tipo "toroidal".

Mostramos na figura 2.4 as possíveis configurações no caso particular do modelo de seis vértices nesta nova geometria, bem como os pesos de Boltzmann correspondentes. Repare que as configurações da primeira fileira são as mesmas que foram apresentadas na figura 1.1, porém agora elas são representadas na rede diagonal ${ }^{2}$. Para simplificar a notação gráfica substituiremos os gráficos da primeira fileira pelos gráficos da segunda fileira desta figura, onde colocamos apenas as flechas cujo sentido aponta para a parte inferior da folha.

\footnotetext{
${ }^{2}$ Os pesos de Boltzmann desta figura representam a exponencial das energias da figura 1.
} 


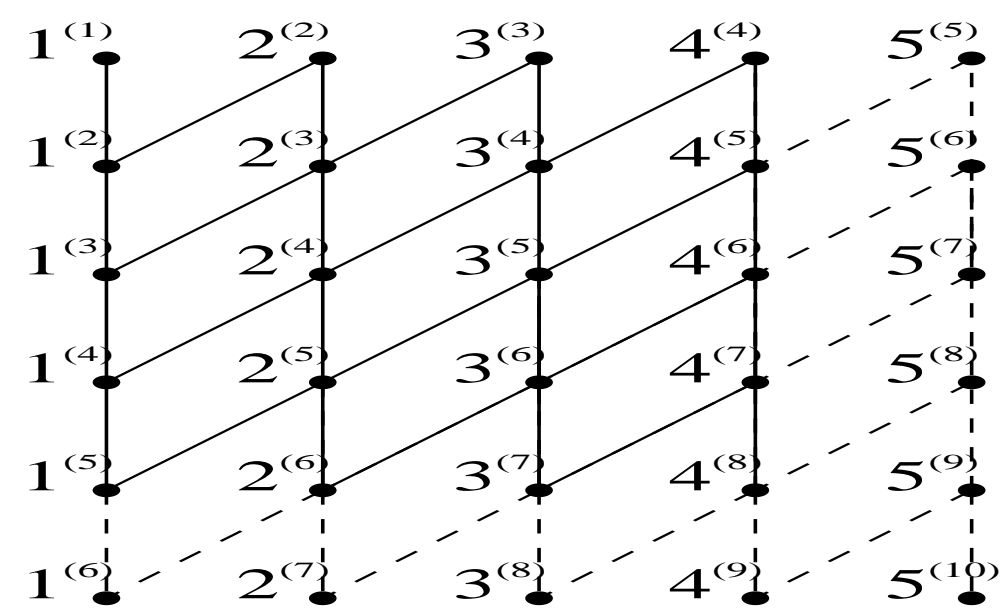

Figura 2.3: Rede diagonal.
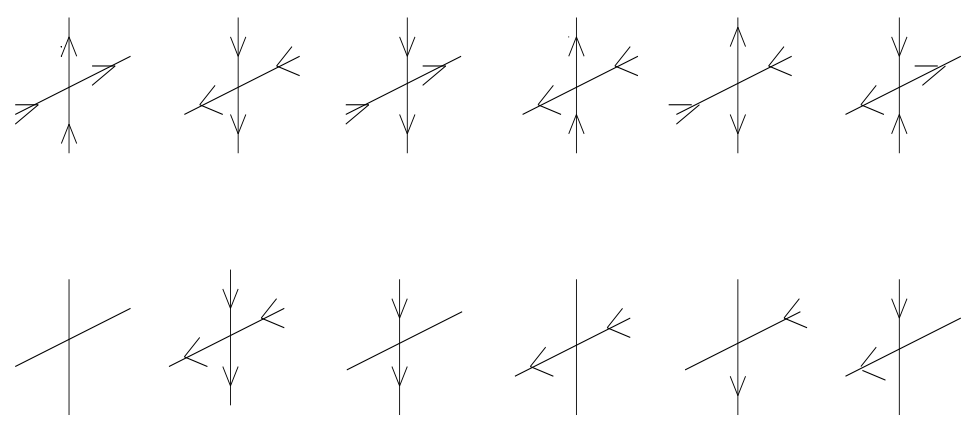

$\mathrm{a}_{0}$

$a_{1}$

$\mathrm{b}_{2}$

$b_{1}$

$c_{1}$

$\mathrm{c}_{2}$

Figura 2.4: Configuração de flechas na rede diagonal.

\subsection{Localização das flechas na rede e a matriz de trans- ferência diagonal-para-diagonal}

Antes de localizarmos as flechas nesta nova rede é fundamental redefinirmos o conceito de linha para esta geometria. As linhas serão representadas de forma tracejada, como pode ser observado na figura 2.5. Chamaremos de sítios os pontos numerados de 1 a $N$, formados pela intersecção das linhas verticais com as linhas inclinadas entre duas linhas sucessivas $r$ e $r+1$ como pode ser observado na figura 2.5 . 


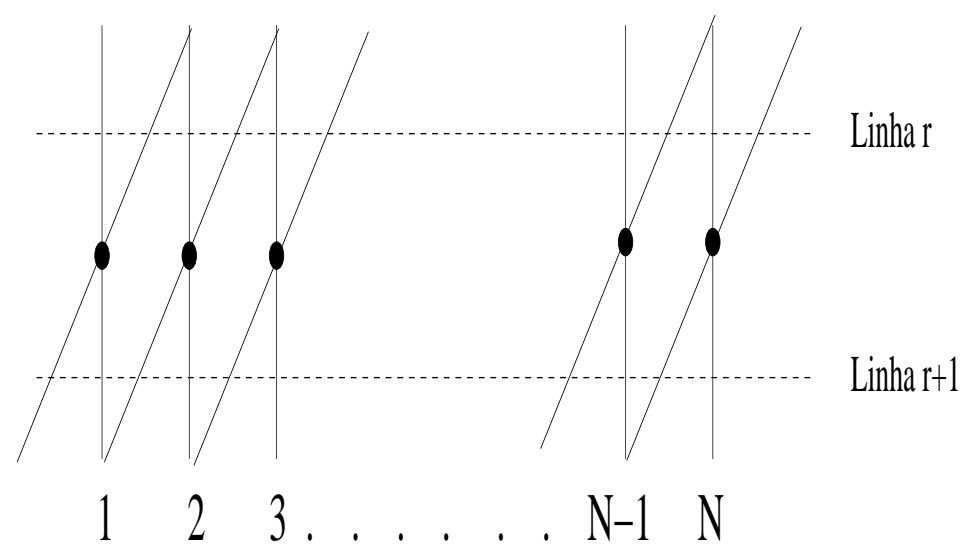

Figura 2.5: Localização das linhas na rede diagonal.

Para localizar um conjunto de $n$ flechas entre duas linhas sucessivas $(r$ e $r+1)$ será necessário especificar não só as posições das flechas $\left(x_{1}, x_{2}, \ldots, x_{n}\right)$, mas também o "estado" $\left(\alpha_{1}, \alpha_{2}, \ldots, \alpha_{n}\right)$ vertical ou inclinado de cada flecha. Designemos então o vetor

$$
\left|\varphi_{r}>\equiv\right| x_{1}, \alpha_{1} ; x_{2}, \alpha_{2} ; \ldots ; x_{n}, \alpha_{n}>
$$

para especificar a configuração de flechas de uma dada linha $r$. O índice $\alpha_{i}(i=$ $1,2, \ldots, n)$ pode assumir dois valores, que correspondem à uma flecha no estado vertical $(\alpha=1)$ ou no estado inclinado $(\alpha=2)$. Como exemplo, exibimos na figura 2.6 a configuração de 4 flechas $\mid 1,1 ; 2,1 ; 2,2 ; 3,2>$ numa rede com $N=4$ sítios, para o modelo de seis vértices.

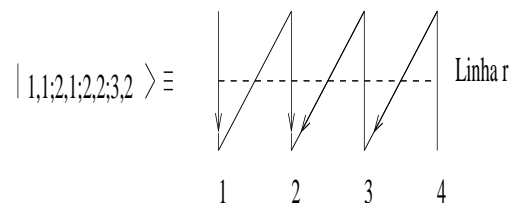

Figura 2.6: Exemplo de uma linha na rede diagonal.

Para calcularmos a função de partição nesta nova geometria deveremos diagonalizar a matriz de transferência $T_{D-D}$ (diagonal-para-diagonal) associada ao modelo, pois 


$$
\begin{aligned}
& Z= \\
& \sum_{\varphi_{1}} \sum_{\varphi_{2}} \ldots \sum_{\varphi_{M}}<\varphi_{1}\left|T_{D-D}\right| \varphi_{2}><\varphi_{2}\left|T_{D-D}\right| \varphi_{3}>\cdots<\varphi_{M}\left|T_{D-D}\right| \varphi_{1}>= \\
& \operatorname{Tr}\left(T_{D-D}^{M}\right) .
\end{aligned}
$$

O elemento da matriz de transferência $<\varphi_{r}\left|T_{D-D}\right| \varphi_{r+1}>$ conecta a configuração $\mid \varphi_{r}>$ da linha $r$ com a configuração $\mid \varphi_{r+1}>$ da linha $r+1$. Para o modelo de 6 vértices este elemento de matriz pode ser escrito como

$$
<\varphi_{r}\left|T_{D-D}\right| \varphi_{r+1}>=a_{0}^{m_{1}} a_{1}^{m_{2}} b_{1}^{m_{3}} b_{2}^{m_{4}} c_{1}^{m_{5}} c_{2}^{m_{6}},
$$

onde o conjunto $\left\{m \equiv m_{1}, m_{2}, \ldots, m_{6}\right\}$ de inteiros contabilizam o número de vértices entre os estados $\mid \varphi_{r}>$ e $\left|\varphi_{r+1}\right\rangle$, e satisfazem o vínculo $\sum_{i=1}^{6} m_{i}=N$. 


\section{Capítulo 3}

\section{Modelos de 5 vértices interagentes}

Os modelos que iremos tratar neste capítulo são definidos na rede quadrada, e os vértices associados aos modelos são mostrados na figura $3.1 \mathrm{com}$ as respectivas fugacidades. Repare que estes vértices são os mesmos da figura 2.4, exceto pela inexistência do vértice de fugacidade $a_{1}$.

Além da interação entre os vértices mais próximos devido à regra do gelo, existem outras interaçôes entre vértices vizinhos ao longo de uma das diagonais. Iremos inicialmente explicar uma versão simplificada dos modelos, para em seguida apresentar o modelo mais geral de 5 vértices abordado em nosso trabalho.
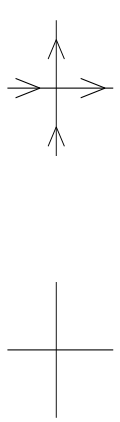

$\mathrm{a} 0$
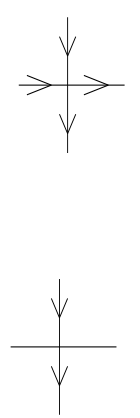

$\mathrm{b}_{2}$
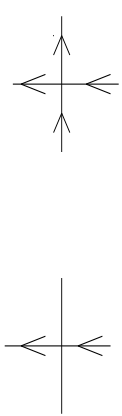

$\mathrm{b}_{1}$
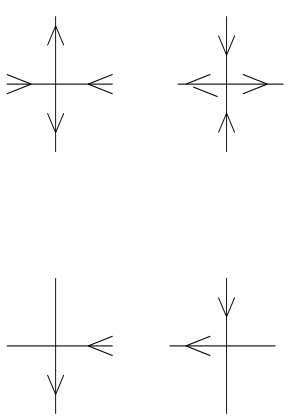

$\mathrm{c}_{1}$

$\mathrm{c}_{2}$

Figura 3.1: Vértices do modelo de 5 vértices. 


\subsection{Modelo de 5 vértices com domínio de interação $s$ fixo}

Além das interações devido à regra do gelo, este modelo possui também interações cujo range depende de um parâmetro inteiro ímpar $s(s=1,3,5 \ldots)$. Tais interações ocorrem ao longo da diagonal que vai do canto superior esquerdo da rede ao canto inferior direito da mesma ${ }^{1}$. Neste modelo as interações entre os vértices não são homogêneas. A distância de interação entre dois vértices ao longo da diagonal de interação éd $=l \sqrt{2} a,(l=1,2, \ldots)$, sendo $a$ o parâmetro da rede. A energia de interação irá depender da distância $D$ (na diagonal) entre os vértices da seguinte maneira:

a) Os vértices não interagem entre si se $D>d$.

b) Se um dos vértices for do tipo $a_{0}$ a interação é nula para $D$.

c) Se os vértices pertencem ao conjunto $\left\{b_{2}, b_{1}, c_{1}, c_{2}\right\}$ a interação é infinita se $D \leq d$ exceto quando os dois vértices são $c_{1}$ e $c_{2}$, estando $c_{2}$ à esquerda superior de $c_{1}$, e os mesmos distarem de $D=d=l \sqrt{2} a$. A energia neste caso é $e_{I}$, sendo o peso de Boltzmann correspondente expresso por conveniência em termos das fugacidades $c_{1}$, $c_{2}$ e de um novo parâmetro que chamanos de $a_{1}$, isto é,

$$
c_{I}=e^{-e_{I}}=\frac{a_{1}}{c_{1} c_{2}}
$$

Ilustramos na figura 3.2 algumas configurações proibidas e permitidas para o caso onde o parâmetro do modelo é $l=1$.

O cálculo da matriz de transferência linha-para-linha embora factível não é simples para o modelo presente, devido às interações não homogêneas entre os vértices na diagonal de interação. Entretanto, quando estudamos a matriz de transferência diagonal-para-diagonal associada ao modelo, as interações entre os vértices da diagonal de interação envolvem apenas as linhas de entrada e saída da matriz, sendo

\footnotetext{
${ }^{1}$ Por simplicidade chamaremos tal diagonal de diagonal de interação
} 

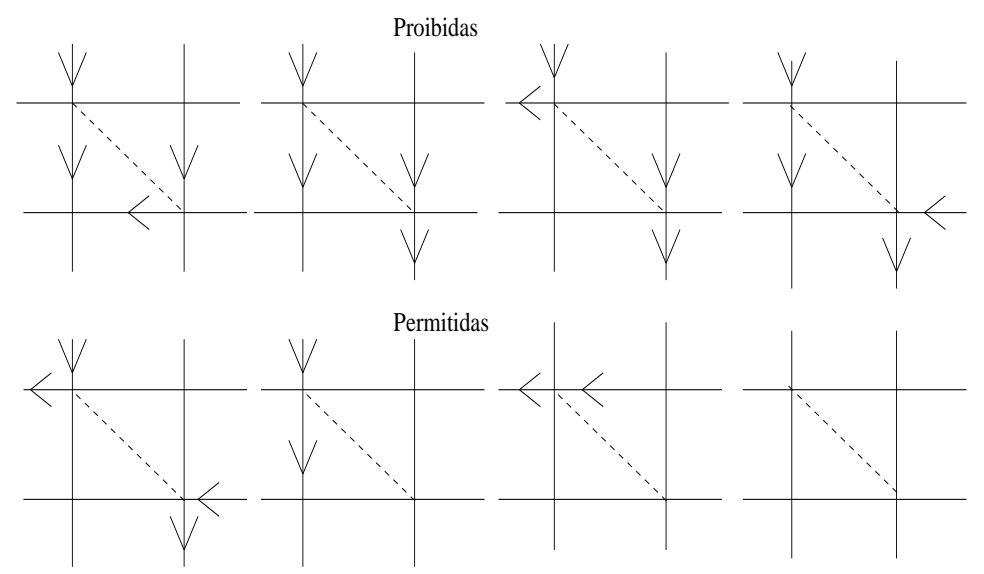

Figura 3.2: Interação entre os vértices à distância $d=a(2)^{\frac{1}{2}}$ ao longo da diagonal para o modelo com $l=1$ (linha para linha).

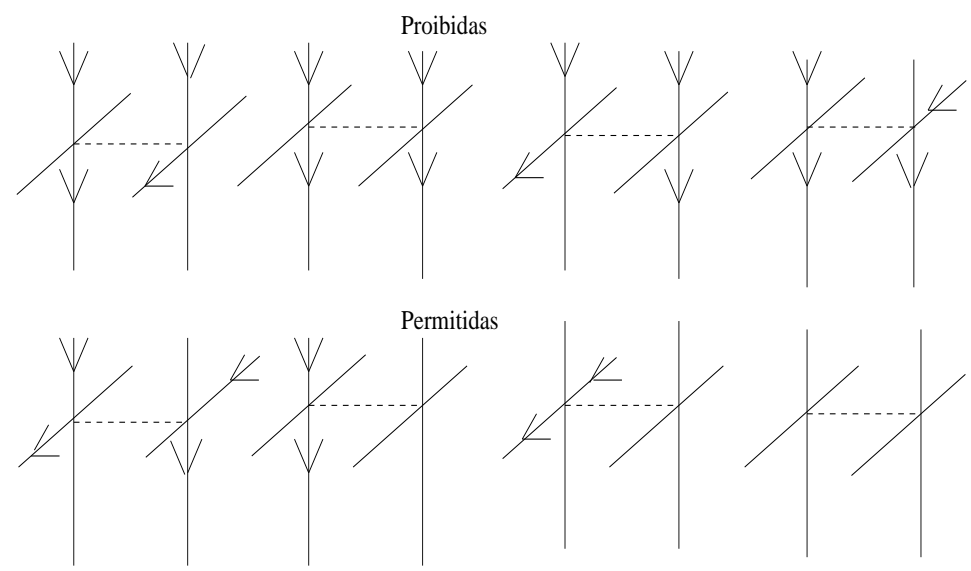

Figura 3.3: Interação entre os vértices à distância $d=a(2)^{\frac{1}{2}}$ ao longo da diagonal para o modelo $\operatorname{com} l=1$ (diagonal-para-diagonal).

assim, a matriz é facilmente calculada. Como exemplo mostramos na figura 3.3 as interações mostradas na figura 3.2 para a rede diagonal.

Na figura 3.4(a) e 3.4(b) mostramos configurações típicas de flechas ao longo da diagonal para os casos $s=2$ e $s=3$, respectivamente. Repare que na figura $3.4 \mathrm{o}$ parâmetro $l$ que determina o modelo, parametriza também o "tamanho" efetivo das flechas, isto é, a distância mínima permitida entre as flechas ao longo da diagonal. 


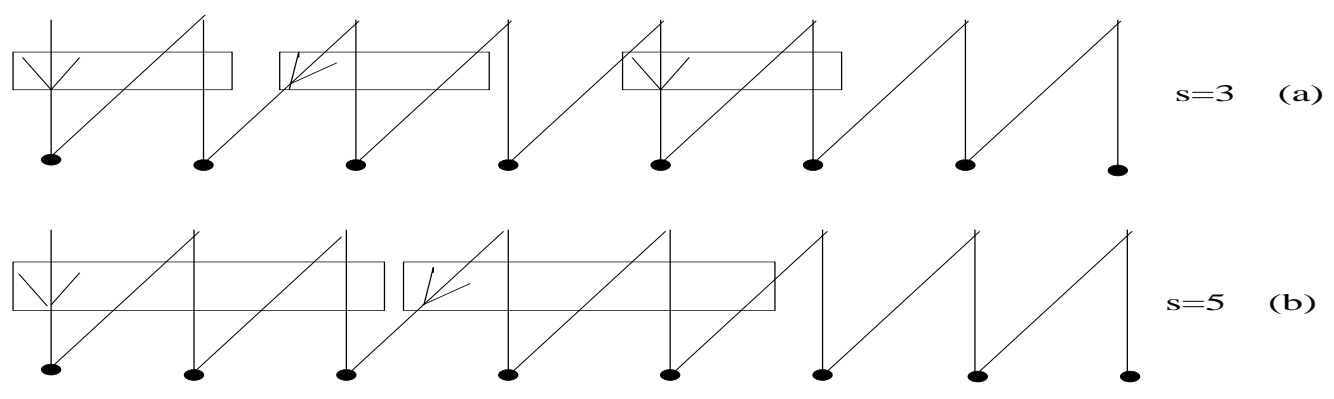

Figura 3.4: Configurações de flechas com tamanho $s=3$ e $s=5$ na rede diagonal.

Mais especificamente uma dada flecha teria um volume de exclusão de $2 l+1$ linhas (verticais ou inclinadas), isto é, a presença de uma dada flecha em uma dada linha (vertical ou inclinada) exclui a presença de outra flecha na linha em questão, bem como nas $2 l$ linhas (vertical ou inclinada) à sua direita.

Nesta formulação de flechas com tamanho efetivo $s$ (ímpar), a extensão do modelo para o caso $s=1$, seria um modelo em que as flechas poderiam ocupar linhas vizinhas. Levando-se em conta a fugacidade dos vértices e a energia de interação (3.1) nos daria um modelo com um vértice adicional (veja figura 3.5) de peso de boltzmann

$$
e^{-e_{I}} c_{1} c_{2}=\frac{a_{1}}{c_{1} c_{2}} c_{1} c_{2}=a_{1}
$$

Assim o modelo com $s=1$ recai no modelo de 6 vértices tradicional constituido apenas das interações de vizinhos próximos imposta pela regra do gelo.

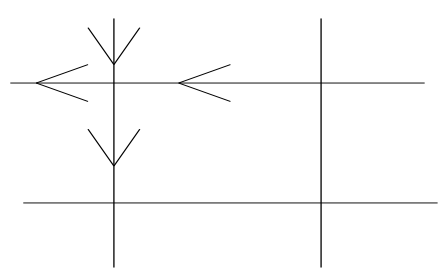

Figura 3.5: Interação entre os vértices $c_{2}$ e $c_{1}$ no modelo com $s=1$. 


\subsection{Modelo de 5 vértices com domínio de interação $s$ variável}

Este modelo é uma versão mais geral do modelo anterior, pois agora, as $n$ flechas em uma dada configuração ao longo da diagonal, e que caracterizam os vértices, ao invés de possuírem um tamanho fixo $s$ poderão possuir tamanhos arbitrários e distintos $\left(s_{1}, s_{2}, \ldots, s_{n}\right)$. Mostramos como exemplo na figura 3.6 a configuração de uma linha contendo três flechas de tamanhos $s=3,1$ e 5 . As flechas se excluem de acordo com seu tamanho e a interação entre duas flechas de tamanho $s_{1}$ e $s_{2}$ é dada por (3.1) caso a flecha de tamanho $s_{2}$ esteja $\left(2 l_{1}+1\right)$ linhas à direita da flecha de tamanho $s_{1}$. O peso de Boltzmann referente às configurações das linhas $r$ e $r^{\prime}$ da figura 3.6 é dada por $c_{2} c_{1} e^{-e_{I}} b_{2} a_{0}^{N-3}$, onde $N$ é o tamanho da rede.

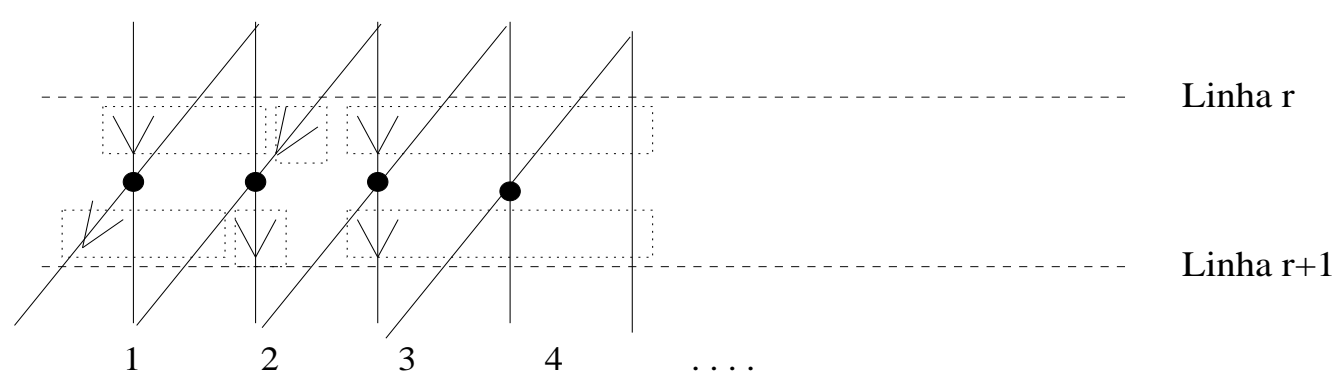

Figura 3.6: Configuração de três flechas com tamanhos $s=3,1$ e 5 .

\subsection{Diagonalização da matriz de transferência diagonal- para-diagonal do modelo de 5 vértices interagentes de domínio de interação $s$ variável}

Devido à conservação do número de flechas na passagem entre duas linhas consecutivas da rede, podemos construir a matriz de transferência na forma de blocos de sub-matrizes disjuntas rotuladas pelo número de flechas. Além desta lei de conservação sabemos que a ordem das flechas na passagem de uma linha para outra 
não é trocada, a menos de permutações cíclicas. Deste modo, a ordem das flechas $\left\{s_{1}, s_{2}, \ldots, s_{n}\right\}$ pode ser considerada um outro bom número quântico. Podemos então subdividir o bloco com $n$ flechas em sub-blocos rotulados por este número quântico adicional $\left\{s_{1}, s_{2}, \ldots, s_{n}\right\}$ (ordenamento das flechas).

Formalmente devemos resolver o seguinte problema de autovalor para um setor envolvendo um número $n$ de flechas, com tamanhos $\left\{s_{1}, s_{2}, \ldots, s_{n}\right\}$

$$
\Lambda_{n}^{\left\{s_{1}, s_{2}, \ldots, s_{n}\right\}}\left|\Psi_{n}^{\left\{s_{1}, s_{2}, \ldots, s_{n}\right\}}>=T_{D-D}^{\left\{s_{1}, s_{2}, \ldots, s_{n}\right\}}\right| \Psi_{n}^{\left\{s_{1}, s_{2}, \ldots, s_{n}\right\}}>
$$

onde $\Lambda_{n}^{\left\{s_{1}, s_{2}, \ldots, s_{n}\right\}}$ e $\mid \Psi_{n}^{\left\{s_{1}, s_{2}, \ldots, s_{n}\right\}}>$ são os autovalores e os autovetores no setor indexado por $n$ flechas e com ordenamento $\left\{s_{1}, s_{2}, \ldots, s_{n}\right\}$. Matematicamente podemos expressar estes autovetores como

$$
\begin{aligned}
& \mid \Psi_{n}^{\left\{s_{1}, s_{2}, \ldots, s_{n}\right\}}>= \\
& \sum_{\{c\}} \sum_{\{x\}} \sum_{\alpha_{1}, \alpha_{2}, \ldots, \alpha_{n}=1}^{2} \phi_{\alpha_{1}, \alpha_{2}, \ldots, \alpha_{n}}^{\left\{s_{c_{1}}, s_{c_{2}}, \ldots, s_{c_{n}}\right\}}\left(x_{1}, x_{2}, \ldots, x_{n}\right) \mid x_{1}, \alpha_{1} ; x_{2}, \alpha_{2} ; \ldots ; x_{n}, \alpha_{n}>.
\end{aligned}
$$

Os kets $\mid x_{1}, \alpha_{1} ; x_{2}, \alpha_{2} ; \ldots ; x_{n}, \alpha_{n}>$ denotam as configurações da rede em que as flechas do tipo $\left(\alpha_{1}, \alpha_{2}, \ldots, \alpha_{n}\right)\left(\alpha_{i}=1,2\right.$ para as flechas verticais e inclinadas) estão localizadas na posição $\{x\}=\left(x_{1}, x_{2}, \ldots, x_{n}\right)$. As funções $\phi_{\alpha_{1}, \alpha_{2}, \ldots, \alpha_{n}}^{\left\{s_{c_{1}}, s_{\left.c_{2}, \ldots, s_{c_{n}}\right\}}\right\}}\left(x_{1}, x_{2}, \ldots, x_{n}\right)$ são as componentes das configurações onde as flechas de tamanho $\left(s_{c_{1}}, s_{c_{2}}, \ldots, s_{c_{n}}\right)$ e do tipo $\left(\alpha_{1}, \alpha_{2}, \ldots, \alpha_{n}\right)$ estão localizadas nas posições $\left(x_{1}, x_{2}, \ldots, x_{n}\right)$, respectivamente. A soma $\{c\}$ extende-se sobre as permutações cíclicas $\left\{c_{1}, c_{2}, \ldots, c_{n}\right\}$ dos inteiros $\{1,2, \ldots, n\}$, e a soma em $\{x\}$ se extende para uma dada distribuição $\left\{s_{c_{1}}, s_{c_{2}}\right.$, $\left.\ldots, s_{c_{n}}\right\}$ de flechas satisfazendo ${ }^{2}$

\footnotetext{
${ }^{2}$ Repare que a relação (3.5) dá conta das possíveis configurações em que a posição $\left(x_{j}\right)$ de uma flecha $j$ coincida com a posição $\left(x_{j+1}\right)$ de uma flecha $j+1$, isto é $x_{j}=x_{j+1}$. Para estas situações temos que ter $\left(\alpha_{j}, \alpha_{j+1}\right)=(1,2)$.
} 


$$
1 \leq x_{1}+s_{1}-1<x_{2}+\delta_{\alpha_{1}, 1} \delta_{\alpha_{2}, 2}<\ldots x_{n-1}+s_{n-1}-1<x_{n}+\delta_{\alpha_{1}, 1} \delta_{\alpha_{2}, 2} \leq N
$$

O ansatz matricial introduzido em [8], apropiado ao presente caso, consiste em propor que tais amplitudes possam ser escritas como o traço de um produto de matrizes. O produto de matrizes associado à configuração $\left\{x_{1}, \alpha_{1} ; x_{2}, \alpha_{2} ; \ldots ; x_{n}, \alpha_{n}\right\}$ é obtido associando-se matrizes às ocupações dos sítios da seguinte forma:

1) Aos sítios sem flechas associamos as matrizes $E$.

2) Aos sítios ocupados por uma uúnica flecha de tamanho $s$ e do tipo $\alpha(\alpha=1,2)$ associamos a matriz $A_{s}^{(\alpha)}$.

3) Aos sítios contendo uma flecha de tamanho $s_{1}=1$ na vertical e outra de tamanho $s_{2}$ inclinado associamos o produto $A_{1}^{(1)} E^{-1} A_{s_{2}}^{(2)}$. Na figura 3.7 abaixo mostramos tais associações. O ansatz matricial no presente caso consiste em assumir

$$
\begin{aligned}
& \phi_{\alpha_{1}, \alpha_{2}, \ldots, \alpha_{n}}^{\left\{s_{1}, s_{2}, \ldots, s_{n}\right\}}\left(x_{1}, x_{2}, \ldots, x_{n}\right)= \\
& \operatorname{Tr}\left[E^{x_{1}-1} A_{s_{1}}^{\left(\alpha_{1}\right)} E^{x_{2}-x_{1}-1} A_{s_{2}}^{\left(\alpha_{2}\right)} \cdots E^{x_{n}-x_{n-1}} A_{s_{n}}^{\left(\alpha_{n}\right)} E^{N-x_{n}} \Omega_{P}\right] .
\end{aligned}
$$

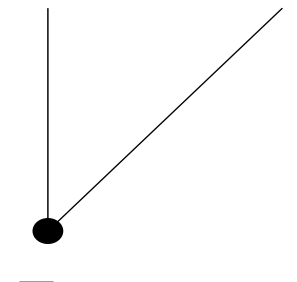

$\mathrm{E}$

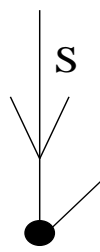

$\mathrm{A}_{\mathrm{S}}^{(1)}$

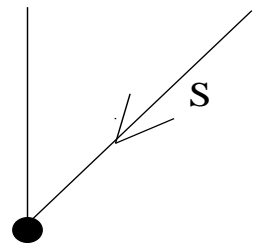

$A \stackrel{(2)}{(2)}$

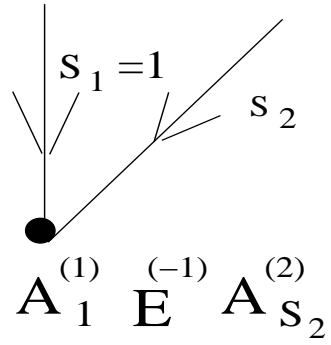

Figura 3.7: Associação dos sítios com as matrizes do ansatz matricial.

Diferentemente do ansatz de Bethe de coordenadas onde $\phi_{\alpha_{1}, \alpha_{2}, \ldots, \alpha_{n}}^{s_{1}, s_{2}, \ldots, s_{n}}\left(x_{1}, x_{2}, \ldots, x_{n}\right)$ é dado como uma combinação linear de ondas planas, aqui, neste novo ansatz, as amplitudes são dadas como o traço de um produto de matrizes. 
Na geometria toroidal que estamos considerando, os auto-estados de $T_{D-D}$ possuem momento $P=\frac{2 \pi j}{N}(j=0,1, \ldots, N-1)$ bem definido. A matriz $T_{D-D}$ pode então ser decomposta em setores disjuntos indexados pelo valor de $P$. A matriz $\Omega_{P}$ é introduzida para assegurar o momento $P$ do autovetor do correspondente setor. Devido à simetria de translação as amplitudes em (3.6) devem satisfazer

$$
\frac{\phi_{\alpha_{1}, \alpha_{2}, \ldots, \alpha_{n}}^{s_{1}, s_{2}, \ldots, s_{n}}\left(x_{1}, x_{2}, \ldots, x_{n}\right)}{\phi_{\alpha_{1}, \alpha_{2}, \ldots, \alpha_{n}}^{s_{1}, s_{2}, \ldots,,_{n}}\left(x_{1}+m, x_{2}+m, \ldots, x_{n}+m\right)}=e^{-i m P}, \quad m=1, \ldots, N-1
$$

o que nos fornece as seguintes relações de comutação entre as matrizes $E, A_{s_{j}}^{\left(\alpha_{j}\right)}$ $\left(\alpha_{j}=1,2\right) \operatorname{com} \Omega_{P}$ :

$$
E \Omega_{P}=e^{-i P} \Omega_{P} E, \quad A_{s_{j}}^{\left(\alpha_{j}\right)} \Omega_{P}=e^{-i P} \Omega_{P} A_{s_{j}}^{\left(\alpha_{j}\right)} .
$$

As relações algébricas entre $A_{s_{j}}^{\left(\alpha_{j}\right)}$ e $E$ serão fixadas no momento que impusermos que $\mid \Psi_{n}^{\left\{s_{1}, s_{2}, \ldots, s_{n}\right\}}>$ satisfaça à equação de autovalor.

Mostraremos a seguir o processo de diagonalização dos setores com $n=0,1,2$ flechas, e em seguida exibiremos o resultado para um valor arbitrário de flechas.

- Setor com $n=0$ flechas

Este setor é uma matriz de tamanho 1 por 1 pois temos apenas uma única configuração, cujo peso de Boltzmann nos dá o autovalor

$$
\Lambda_{0}=a_{0}^{N}
$$

- Setor com $n=1$ flecha

Temos neste subespaço $2 N$ possíveis configurações. A equação de autovalor produzirá relações a serem satisfeitas entre as $2 N$ amplitudes correspondentes às diversas configurações de flechas. 
Deveremos observar que devido aos dois "estados" possíveis para uma determinada flecha (vertical ou inclinado), tais relações são dadas pelo conjunto de $2 N$ equações

$$
\begin{aligned}
& \Lambda_{1} \operatorname{Tr}\left[E^{x-1} A_{s_{1}}^{(1)} E^{N-x} \Omega_{P}\right]= \\
& b_{2} a_{0}^{N-1} \operatorname{Tr}\left[E^{x-1} A_{s_{1}}^{(1)} E^{N-x} \Omega_{P}\right]+c_{1} a_{0}^{N-1} \operatorname{Tr}\left[E^{x-1} A_{s_{1}}^{(2)} E^{N-x} \Omega_{P}\right], \\
& \Lambda_{1} \operatorname{Tr}\left[E^{x-1} A_{s_{1}}^{(2)} E^{N-x} \Omega_{P}\right]= \\
& c_{2} a_{0}^{N-1} \operatorname{Tr}\left[E^{x} A_{s_{1}}^{(1)} E^{N-x-1} \Omega_{P}\right]+b_{1} a_{0}^{N-1} \operatorname{Tr}\left[E^{x} A_{s_{1}}^{(2)} E^{N-x-1} \Omega_{P}\right] .
\end{aligned}
$$

Nas figuras 3.8 e 3.9 representamos pictoricamente tais equações. As equações (3.10) são resolvidas se expressarmos

$$
\begin{aligned}
& A_{s_{1}}^{(1)}=\phi_{1} A_{k}^{s_{1}} E^{2-s_{1}}, \\
& A_{s_{1}}^{(2)}=\phi_{2} A_{k}^{s_{1}} E^{2-s_{1}},
\end{aligned}
$$

sendo $A_{k}^{s_{1}}$ uma matriz que depende de um parâmetro escalar $k$ (parâmetro espectral) e $\phi_{i},(i=1,2)$ constantes reais. Substituindo-se (3.11) na primeira das equações de (3.10) obtemos

$$
\begin{aligned}
& \Lambda_{1} \operatorname{Tr}\left[E^{x-1} \phi_{1} A_{k}^{s_{1}} E^{2-s_{1}} E^{N-x} \Omega_{P}\right]= \\
& b_{2} a_{0}^{N-1} \operatorname{Tr}\left[E^{x-1} \phi_{1} A_{k}^{s_{1}} E^{2-s_{1}} E^{N-x} \Omega_{P}\right]+c_{1} a_{0}^{N-1} \operatorname{Tr}\left[E^{x-1} \phi_{2} A_{k}^{s_{1}} E^{2-s_{1}} E^{N-x} \Omega_{P}\right] .
\end{aligned}
$$

Fatorando-se o termo comum na expressão acima obtemos,

$$
\left(\Lambda_{1} \phi_{1}-b_{2} a_{0}^{N-1} \phi_{1}-c_{1} a_{0}^{N-1} \phi_{2}\right) \operatorname{Tr}\left[E^{x-1} A_{k}^{s_{1}} E^{2-s_{1}} E^{N-x} \Omega_{P}\right]=0 .
$$


Impondo que $\operatorname{Tr}\left[E^{x-1} A_{k}^{s_{1}} E^{2-s_{1}} E^{N-x} \Omega_{P}\right] \neq 0$ obtemos

$$
\Lambda_{1} \phi_{1}-b_{2} a_{2}^{N-1} \phi_{1}-c_{1} a_{2}^{N-1} \phi_{2}=0
$$

Por outro lado, substituindo-se (3.11) na segunda das equações de (3.10) obtemos

$$
\begin{aligned}
& \Lambda_{1} \operatorname{Tr}\left[E^{x-1} \phi_{2} A_{k}^{s_{1}} E^{2-s_{1}} E^{N-x} \Omega_{P}\right]= \\
& c_{2} a_{0}^{N-1} \operatorname{Tr}\left[E^{x} \phi_{1} A_{k}^{s_{1}} E^{2-s_{1}} E^{N-x-1} \Omega_{P}\right]+b_{1} a_{0}^{N-1} \operatorname{Tr}\left[E^{x} \phi_{2} A_{k}^{s_{1}} E^{2-s_{1}} E^{N-x-1} \Omega_{P}\right] .
\end{aligned}
$$

Repare que esta relação se simplifica se impormos que a matriz $A_{k}^{s_{1}}$ introduzida em (3.11) satisfaça à relação de comutação

$$
E A_{k}^{s_{1}}=e^{i k} A_{k}^{s_{1}} E
$$

o que nos dá

$$
\left(\Lambda_{1} \phi_{2}-c_{2} a_{0}^{N-1} \phi_{1} e^{i k}-b_{1} a_{0}^{N-1} \phi_{2} e^{k}\right) \operatorname{Tr}\left[E^{x-1} A_{k}^{s_{1}} E^{2-s_{1}} E^{N-x} \Omega_{P}\right]=0 .
$$

Impondo que $\operatorname{Tr}\left[E^{x-1} A_{k}^{s_{1}} E^{2-s_{1}} E^{N-x} \Omega_{P}\right] \neq 0$ obtemos

$$
\Lambda_{1} \phi_{2}-c_{2} a_{0}^{N-1} \phi_{1} e^{k}-b_{1} a_{0}^{N-1} \phi_{2} e^{i k}=0
$$

Vemos assim que as $(2 N)$ equações (3.10) reduzem-se somente à duas equações (3.14) e (3.18), que podem ser reescritas na forma matricial

$$
\frac{\Lambda_{1}}{a_{0}^{N-1}}\left(\begin{array}{l}
\phi_{1} \\
\phi_{2}
\end{array}\right)=\left(\begin{array}{cc}
b_{2} & c_{1} \\
c_{2} e^{i k} & b_{1} e^{i k}
\end{array}\right)\left(\begin{array}{l}
\phi_{1} \\
\phi_{2}
\end{array}\right) .
$$




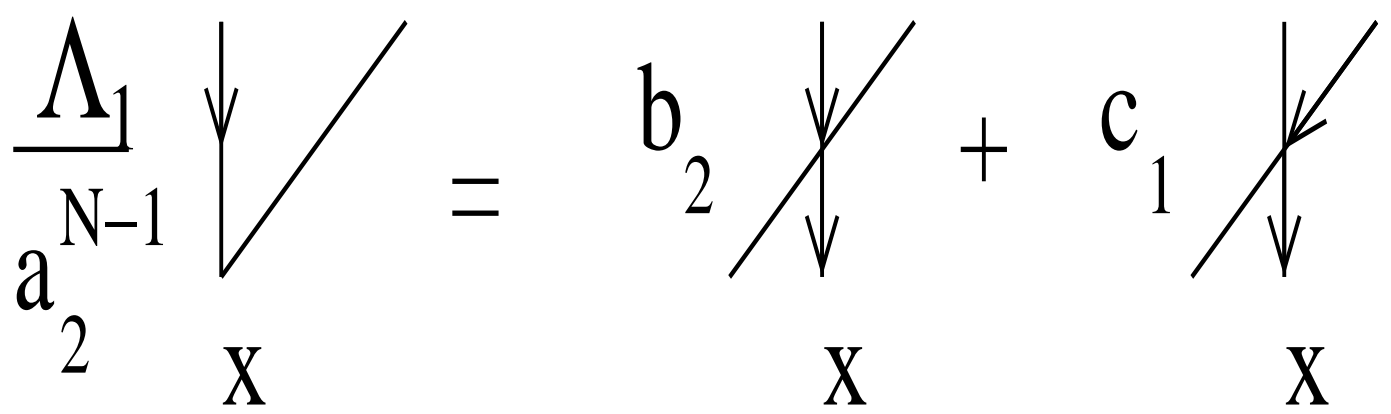

Figura 3.8: Representação pictórica da equação de autovalor (situação 1)

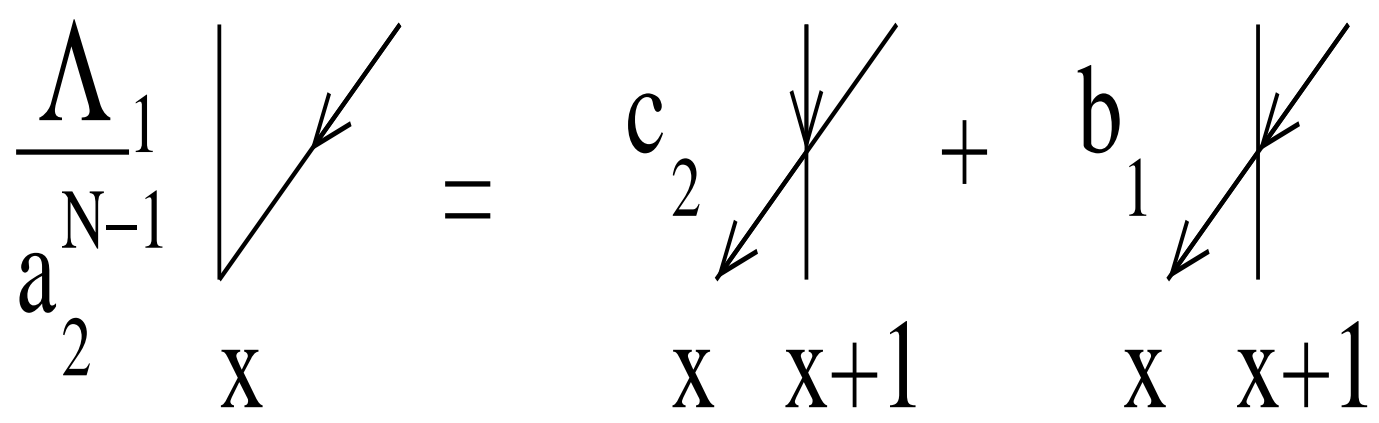

Figura 3.9: Representação pictórica da equação de autovalor (situação 2)

Diagonalizando esta matriz obtemos os dois autovalores $\left\{\Lambda_{1}^{(l)} ; l=-,+\right\}$ :

$$
\Lambda_{1}^{(l)}(k)=\frac{a_{0}^{N-1}}{2}\left(b_{2}+b_{1} e^{i k}+l\left[\left(b_{2}+b_{1} e^{i k}\right)^{2}-4 e^{i k}\left(b_{2} b_{1}-c_{2} c_{1}\right)\right]^{\frac{1}{2}}\right)
$$

Para completarmos a diagonalização resta-nos determinar o parâmetro espectral $k$. Tal parâmetro no presente caso coincide com $P$, pois usando-se $(3.11),(3.16),(3.8)$ e a propriedade cíclica do traço temos

$$
\begin{aligned}
& \operatorname{Tr}\left[E^{x-1} A_{s_{1}}^{(j)} E^{N-x} \Omega_{P}\right]=\operatorname{Tr}\left[E^{x-1} E^{N-x} A_{s_{1}}^{(j)} \Omega_{P}\right] e^{-i k(N-x)}= \\
& \operatorname{Tr}\left[E^{x-1} E^{N-x} \Omega_{P} A_{s_{1}}^{(j)}\right] e^{-i k(N-x)} e^{-i P}= \\
& \operatorname{Tr}\left[E^{x-1} A_{s_{1}}^{(j)} E^{N-x} \Omega_{P}\right] e^{-i k(N-x)} e^{-i P} e^{-i k(x-1)}
\end{aligned}
$$

o que implica 


$$
k=P=\frac{2 m \pi}{N}, \quad m=0,1, \ldots, N-1
$$

Tais valores determinam os $2 N$ autovalores dados em (3.20).

- Setor com $n=2$ flechas

Vamos primeiramente analisar as equações provenientes das configurações $\mid x_{1}, \alpha_{1} ; x_{2}, \alpha_{2}>$ em que as flechas localizadas nas posições $x_{1}$ e $x_{2}$ não estejam em colisão $\left(\mid x_{1}, \alpha_{1} ; x_{2}, \alpha_{2}>\neq\right.$ $\left.\mid x_{1}, 2 ; x_{2}=x_{1}+s_{1}>\right)$. As equações obtidas são

$$
\begin{aligned}
& \Lambda_{2} \operatorname{Tr}\left[E^{x_{1}-1} A_{s_{1}}^{(1)} E^{x_{2}-x_{1}-1} A_{s_{2}}^{(1)} E^{N-x_{2}} \Omega_{P}\right] / a_{0}^{N-2}= \\
& b_{2}^{2} \operatorname{Tr}\left[E^{x_{1}-1} A_{s_{1}}^{(1)} E^{x_{2}-x_{1}-1} A_{s_{2}}^{(1)} E^{N-x_{2}} \Omega_{P}\right]+c_{1} b_{2} \operatorname{Tr}\left[E^{x_{1}-1} A_{s_{1}}^{(2)} E^{x_{2}-x_{1}-1} A_{s_{2}}^{(1)} E^{N-x_{2}} \Omega_{P}\right]+ \\
& b_{2} c_{1} \operatorname{Tr}\left[E^{x_{1}-1} A_{s_{1}}^{(1)} E^{x_{2}-x_{1}-1} A_{s_{2}}^{(2)} E^{N-x_{2}} \Omega_{P}\right]+c_{1}^{2} \operatorname{Tr}\left[E^{x_{1}-1} A_{s_{1}}^{(2)} E^{x_{2}-x_{1}-1} A_{s_{2}}^{(2)} E^{N-x_{2}} \Omega_{P}\right],
\end{aligned}
$$

$\Lambda_{2} \operatorname{Tr}\left[E^{x_{1}-1} A_{s_{1}}^{(1)} E^{x_{2}-x_{1}-1} A_{s_{2}}^{(2)} E^{N-x_{2}} \Omega_{P}\right] / a_{0}^{N-2}=$

$c_{2} b_{2} \operatorname{Tr}\left[E^{x_{1}-1} A_{s_{1}}^{(1)} E^{x_{2}-x_{1}} A_{s_{2}}^{(1)} E^{N-x_{2}-1} \Omega_{P}\right]+c_{1} c_{2} \operatorname{Tr}\left[E^{x_{1}-1} A_{s_{1}}^{(2)} E^{x_{2}-x_{1}} A_{s_{2}}^{(1)} E^{N-x_{2}-1} \Omega_{P}\right]+$ $b_{2} b_{1} \operatorname{Tr}\left[E^{x_{1}-1} A_{s_{1}}^{(1)} E^{x_{2}-x_{1}} A_{s_{2}}^{(2)} E^{N-x_{2}-1} \Omega_{P}\right]+c_{1} b_{1} \operatorname{Tr}\left[E^{x_{1}-1} A_{s_{1}}^{(2)} E^{x_{2}-x_{1}} A_{s_{2}}^{(2)} E^{N-x_{2}-1} \Omega_{P}\right]$,

$\Lambda_{2} \operatorname{Tr}\left[E^{x_{1}-1} A_{s_{1}}^{(2)} E^{x_{2}-x_{1}-1} A_{s_{2}}^{(1)} E^{N-x_{2}} \Omega_{P}\right] / a_{0}^{N-2}=$

$c_{2} b_{2} \operatorname{Tr}\left[E^{x_{1}} A_{s_{1}}^{(1)} E^{x_{2}-x_{1}-2} A_{s_{2}}^{(1)} E^{N-x_{2}} \Omega_{P}\right]+b_{1} b_{2} \operatorname{Tr}\left[E^{x_{1}} A_{s_{1}}^{(2)} E^{x_{2}-x_{1}-2} A_{s_{2}}^{(1)} E^{N-x_{2}} \Omega_{P}\right]+$ $c_{2} c_{1} \operatorname{Tr}\left[E^{x_{1}} A_{s_{1}}^{(1)} E^{x_{2}-x_{1}-2} A_{s_{2}}^{(2)} E^{N-x_{2}} \Omega_{P}\right]+b_{1} c_{1} \operatorname{Tr}\left[E^{x_{1}} A_{s_{1}}^{(2)} E^{x_{2}-x_{1}-2} A_{s_{2}}^{(2)} E^{N-x_{2}} \Omega_{P}\right]$,

$\Lambda_{2} \operatorname{Tr}\left[E^{x_{1}-1} A_{s_{1}}^{(2)} E^{x_{2}-x_{1}-1} A_{s_{2}}^{(2)} E^{N-x_{2}} \Omega_{P}\right] / a_{0}^{N-2}=$

$c_{2}^{2} \operatorname{Tr}\left[E^{x_{1}} A_{s_{1}}^{(1)} E^{x_{2}-x_{1}-1} A_{s_{2}}^{(1)} E^{N-x_{2}-1} \Omega_{P}\right]+b_{1} c_{2} \operatorname{Tr}\left[E^{x_{1}} A_{s_{1}}^{(2)} E^{x_{2}-x_{1}-1} A_{s_{2}}^{(1)} E^{N-x_{2}-1} \Omega_{P}\right]+$ $c_{2} b_{1} \operatorname{Tr}\left[E^{x_{1}} A_{s_{1}}^{(1)} E^{x_{2}-x_{1}-1} A_{s_{2}}^{(2)} E^{N-x_{2}-1} \Omega_{P}\right]+b_{1}^{2} \operatorname{Tr}\left[E^{x_{1}} A_{s_{1}}^{(2)} E^{x_{2}-x_{1}-1} A_{s_{2}}^{(2)} E^{N-x_{2}-1} \Omega_{P}\right]$, 
As visualizaçôes gráficas das equações (3.23)-(3.26) são expostas nas figuras (3.10a) $(3.10 d)$, respectivamente, para o caso especial em que $s_{1}=s_{2}=1$.

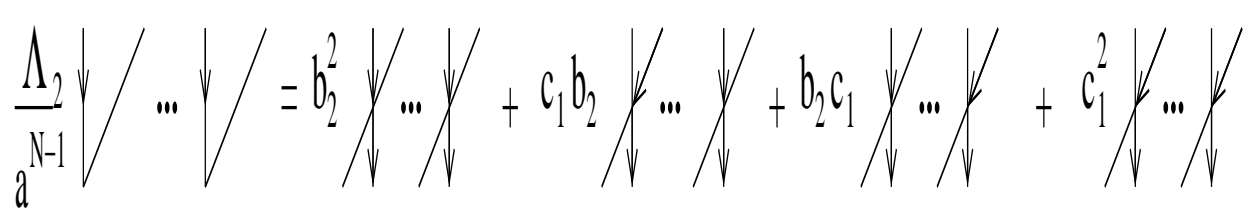

$$
\begin{aligned}
& \begin{array}{lllllllllll}
2 & x_{1} & X_{2} & X_{1} & X_{2} & x_{1} & X_{2} & x_{1} & X_{2} & X_{1} & X_{2}
\end{array}
\end{aligned}
$$

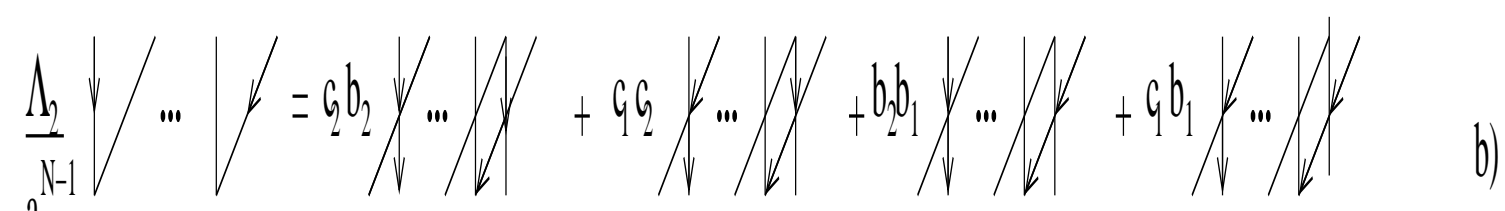

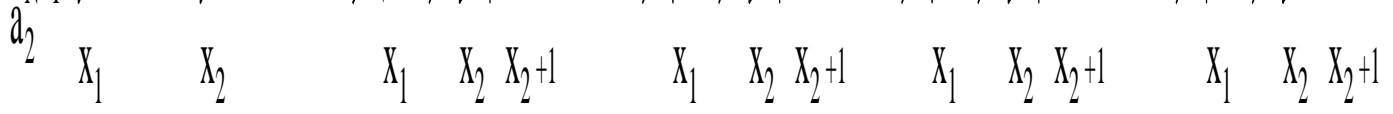

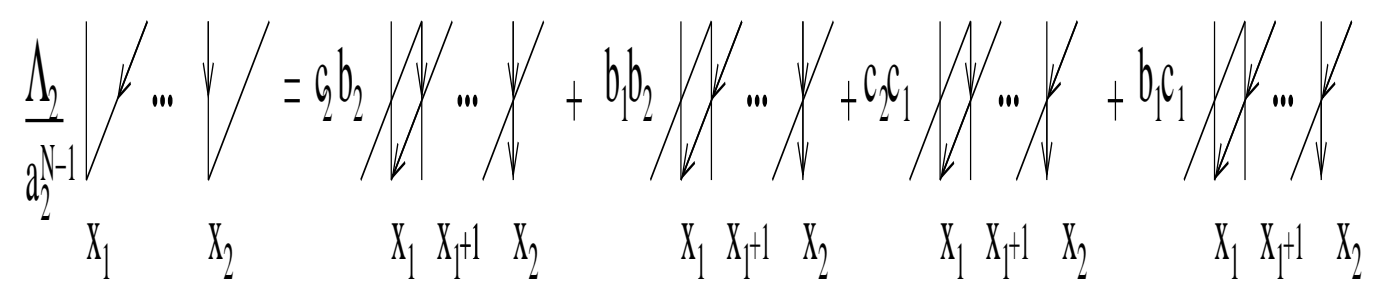

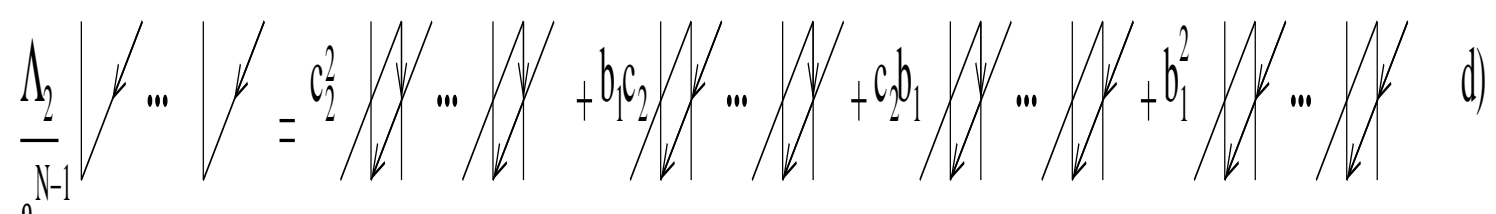

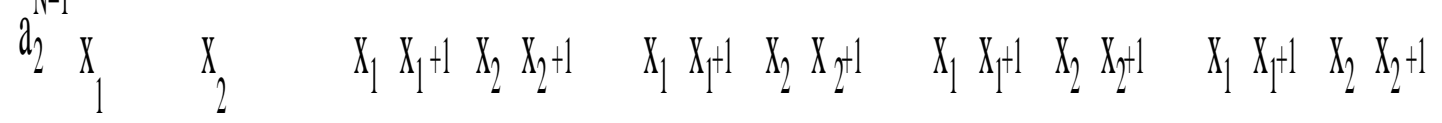

Figura 3.10: Representação gráfica da equação de autovalor para a amplitude advindas advindas de configurações de 2 flechas que não estejam em colisão.

Tais equações são resolvidas mediante uma generalização do ansatz (3.11). As matrizes $A_{s_{1}}^{(1)}, A_{s_{2}}^{(2)}$ são dadas por 


$$
A_{s_{j}}^{(\alpha)}=\sum_{i=1}^{2} \phi_{\alpha}^{i} A_{k_{i}}^{s_{j}} E^{2-s_{j}}, \quad(j=1,2), \quad(\alpha=1,2) .
$$

Assim como no caso de uma flecha $\phi_{\alpha}^{i}(i=1,2)$ são parâmetros escalares e $A_{k_{i}}^{s_{j}}$ $(i, j=1,2)$ são matrizes que satisfazem às seguintes relações de comutação

$$
E A_{k_{i}}^{s_{j}}=e^{i k_{i}} A_{k_{i}}^{s_{j}} E, \quad i, j=1,2 .
$$

Usando-se (3.27) e (3.28) as equações (3.23)-(3.26) tomam, respectivamente, a forma simplificada

$$
\begin{aligned}
& \sum_{i, j=1 ; i \neq j}^{2}\left(\frac{\Lambda_{0}}{a_{0}^{N-2}} \phi_{1}^{i} \phi_{1}^{j}-b_{2}^{2} \phi_{1}^{i} \phi_{1}^{j}-c_{1} b_{2} \phi_{2}^{i} \phi_{1}^{j}-b_{2} c_{1} \phi_{1}^{i} \phi_{2}^{j}-c_{1}^{2} \phi_{2}^{i} \phi_{2}^{j}\right) \\
& \times \operatorname{Tr}\left[E^{x_{1}-1} A_{k_{i}}^{s_{1}} E^{2-s_{1}} E^{x_{2}-x_{1}-1} A_{k_{j}}^{s_{2}} E^{2-s_{2}} E^{N-x_{2}} \Omega_{P}\right]=0,
\end{aligned}
$$

$$
\begin{aligned}
& \sum_{i, j=1 ; i \neq j}^{2}\left(\frac{\Lambda_{0}}{a_{0}^{N-2}} \phi_{1}^{i} \phi_{2}^{j}-b_{2} c_{2} \phi_{1}^{i} \phi_{1}^{j} e^{i k_{j}}-c_{1} c_{2} \phi_{2}^{i} \phi_{1}^{j} e^{i k_{j}}-b_{2} b_{1} \phi_{1}^{i} \phi_{2}^{j} e^{i k_{j}}-c_{1} b_{1} \phi_{2}^{i} \phi_{2}^{j} e^{i k_{j}}\right) \\
& \times \operatorname{Tr}\left[E^{x_{1}-1} A_{k_{i}}^{s_{1}} E^{2-s_{1}} E^{x_{2}-x_{1}-1} A_{k_{j}}^{s_{2}} E^{2-s_{2}} E^{N-x_{2}} \Omega_{P}\right]=0
\end{aligned}
$$

$$
\begin{aligned}
& \sum_{i, j=1 ; i \neq j}^{2}\left(\frac{\Lambda_{0}}{a_{0}^{N-2}} \phi_{2}^{i} \phi_{1}^{j}-b_{2} c_{2} \phi_{1}^{i} \phi_{1}^{j} e^{i k_{i}}-b_{1} b_{2} \phi_{2}^{i} \phi_{1}^{j} e^{i k_{i}}-c_{2} c_{1} \phi_{1}^{i} \phi_{2}^{j} e^{i k_{i}}-c_{1} b_{1} \phi_{2}^{i} \phi_{2}^{j} e^{i k_{i}}\right) \\
& \times \operatorname{Tr}\left[E^{x_{1}-1} A_{k_{i}}^{s_{1}} E^{2-s_{1}} E^{x_{2}-x_{1}-1} A_{k_{j}}^{s_{2}} E^{2-s_{2}} E^{N-x_{2}} \Omega_{P}\right]=0
\end{aligned}
$$

$$
\begin{aligned}
& \sum_{i, j=1 ; i \neq j}^{2}\left(\frac{\Lambda_{0}}{a_{0}^{N-2}} \phi_{2}^{i} \phi_{2}^{j}-c_{2}^{2} \phi_{1}^{i} \phi_{1}^{j} e^{i\left(k_{i}+k_{j}\right)}-b_{1} c_{2} \phi_{2}^{i} \phi_{1}^{j} e^{i\left(k_{i}+k_{j}\right)}-c_{2} b_{1} \phi_{1}^{i} \phi_{2}^{j} e^{i\left(k_{i}+k_{j}\right)}-\right. \\
& \left.b_{1}^{2} \phi_{2}^{i} \phi_{2}^{j} e^{i\left(k_{i}+k_{j}\right)}\right) \times \operatorname{Tr}\left[E^{x_{1}-1} A_{k_{i}}^{s_{1}} E^{2-s_{1}} E^{x_{2}-x_{1}-1} A_{k_{j}}^{s_{2}} E^{2-s_{2}} E^{N-x_{2}} \Omega_{P}\right]=0 .
\end{aligned}
$$


Impondo que a quantidade $\operatorname{Tr}\left[E^{x_{1}-1} A_{k_{i}}^{s_{1}} E^{2-s_{1}} E^{x_{2}-x_{1}-1} A_{k_{j}}^{s_{2}} E^{2-s_{2}} E^{N-x_{2}} \Omega_{P}\right] \neq 0$ para quaisquer valores de $x_{2}$ e $x_{1}$, extraímos as equações algébricas escritas na forma matricial

$$
\begin{aligned}
& \frac{\Lambda_{2}}{a_{0}^{N-2}}\left(\begin{array}{c}
\phi_{1}^{1} \phi_{1}^{2} \\
\phi_{1}^{1} \phi_{2}^{2} \\
\phi_{2}^{1} \phi_{1}^{2} \\
\phi_{2}^{1} \phi_{2}^{2}
\end{array}\right)= \\
& \left(\begin{array}{cccc}
b_{2}^{2} & b_{2} c_{1} & c_{1} b_{2} & c_{1}^{2} \\
b_{2} c_{2} e^{i k_{1}} & b_{2} b_{1} e^{i k_{1}} & c_{1} c_{2} e^{i k_{1}} & c_{1} b_{1} e^{i k_{1}} \\
c_{2} b_{2} e^{i k_{2}} & c_{2} c_{1} e^{i k_{2}} & b_{1} b_{2} e^{i k_{2}} & b_{1} c_{1} e^{i k_{2}} \\
c_{2}^{2} e^{i\left(k_{1}+k_{2}\right)} & c_{2} b_{1} e^{i\left(k_{1}+k_{2}\right)} & b_{1} c_{2} e^{i\left(k_{1}+k_{2}\right)} & b_{1}^{2} e^{i\left(k_{1}+k_{2}\right)}
\end{array}\right)\left(\begin{array}{c}
\phi_{1}^{1} \phi_{1}^{2} \\
\phi_{1}^{1} \phi_{2}^{2} \\
\phi_{2}^{1} \phi_{1}^{2} \\
\phi_{2}^{1} \phi_{2}^{2}
\end{array}\right)
\end{aligned}
$$

É interessante observar que esta última equação pode ser escrita na forma tensorial

$$
\begin{aligned}
& \frac{\Lambda_{2}}{a_{0}^{N-2}}\left(\begin{array}{l}
\phi_{1}^{1} \\
\phi_{2}^{1}
\end{array}\right) \otimes\left(\begin{array}{l}
\phi_{1}^{2} \\
\phi_{2}^{2}
\end{array}\right)= \\
& \left(\begin{array}{cc}
b_{2} & c_{1} \\
c_{2} e^{i k_{2}} & b_{1} e^{i k_{2}}
\end{array}\right) \otimes\left(\begin{array}{cc}
b_{2} & c_{1} \\
c_{2} e^{i k_{1}} & b_{1} e^{i k_{1}}
\end{array}\right)\left(\begin{array}{l}
\phi_{1}^{1} \\
\phi_{2}^{1}
\end{array}\right) \otimes\left(\begin{array}{l}
\phi_{1}^{2} \\
\phi_{2}^{2}
\end{array}\right) .
\end{aligned}
$$

Sem perda de generalidade podemos sempre, escolher um dos pesos de Boltzmann como a unidade. Isto decorre do fato de podermos escolher uma das energias associada aos pesos de Boltzmann como nula. Em função dessa arbitrariedade vamos neste momento fazer $a_{0}=1$. Se escrevermos $\Lambda_{2}=\Lambda_{1}\left(k_{1}\right) \Lambda_{1}\left(k_{2}\right)$, vemos que (3.34) se fatoriza em duas equações. Isto nos mostra que o autovalor da matriz de transferência no setor que contém duas flechas é dado pelo produto dos autovalores de uma única flecha com números de onda $k_{1}$ e $k_{2}$. A relação que fixa estes números de onda será obtida adiante.

É simples verificar usando-se (3.8), (3.27) e (3.28) que as relações de comutação entre as matrizes $A_{k_{j}}^{s_{i}}$ são dadas por 


$$
A_{k_{i}}^{s_{j}} \Omega_{P}=e^{-i P\left(1-s_{j}\right)} \Omega_{P} A_{k_{i}}^{s_{j}}, \quad(j, i=1,2) .
$$

Partimos agora para tentar extrair uma relação do parâmetro $P$ com as variáveis espectrais $k_{1}$ e $k_{2}$. Para isso devemos comparar as componentes de $\mid x_{1}, \alpha_{1} ; x_{2}, \alpha_{2}>$ e $\mid x_{1}+m, \alpha_{1} ; x_{2}+m, \alpha_{2}>$. A razão destas duas amplitutes nos fornece

$$
\frac{\operatorname{Tr}\left[E^{x_{1}-1} A^{\left(\alpha_{1}\right)} E^{x_{2}-x_{1}-1} A^{\left(\alpha_{2}\right)} E^{N-x_{2}} \Omega_{P}\right]}{\operatorname{Tr}\left[E^{x_{1}+m-1} A^{\left(\alpha_{1}\right)} E^{x_{2}-x_{1}-1} A^{\left(\alpha_{2}\right)} E^{N-x_{2}-m} \Omega_{P}\right]}=e^{-i m P} .
$$

Substituindo (3.8) e (3.35) em (3.36) extraímos que

$$
e^{-i\left(k_{i}+k_{j}\right)}=e^{-i m P}, \quad(i, j=1,2) ; i \neq j .
$$

O que implica que o momento $P$, que caracteriza este setor, é dado pela soma das variáveis espectrais $k_{1}$ e $k_{2}$ cujos possíveis valores até neste momento não estão determinados.

Vamos agora estudar as equações adicionais não consideradas em (3.23)-(3.26). Estas equações são provenientes das configurações em que $x_{2}=x_{1}+s_{1} ; \alpha_{1}=2$ e $\alpha_{2}=1$. Esta situação está relacionada com a "colisão" entre duas flechas, que é representada pictoricamente na figura 3.11 abaixo, para o caso especial em que as duas flechas possuem o mesmo tamanho $s_{1}=s_{2}=3$. As equações para as amplitudes referentes a esta situação são dadas por

$$
\begin{aligned}
& \Lambda_{2} \operatorname{Tr}\left[E^{x_{1}-1} A_{s_{1}}^{(2)} E^{x_{1}+s_{1}-x_{1}-1} A_{s_{2}}^{(1)} E^{N-x_{1}-s_{1}} \Omega_{P}\right]= \\
& c_{1} c_{2} c_{I} \operatorname{Tr}\left[E^{x_{1}} A_{s_{1}}^{(1)} E^{x_{1}+s_{1}-x_{1}-1-1} A_{s_{2}}^{(2)} E^{N-x_{1}-s_{1}} \Omega_{P}\right]= \\
& a_{1} \operatorname{Tr}\left[E^{x_{1}} A_{s_{1}}^{(1)} E^{x_{1}+s_{1}-x_{1}-1-1} A_{s_{2}}^{(2)} E^{N-x_{1}-s_{1}} \Omega_{P}\right],
\end{aligned}
$$

onde usou-se o fato de $c_{I}=\frac{a_{1}}{c_{1} c_{2}}$. Substituindo (3.27) e (3.28) em (3.38) extraímos que 


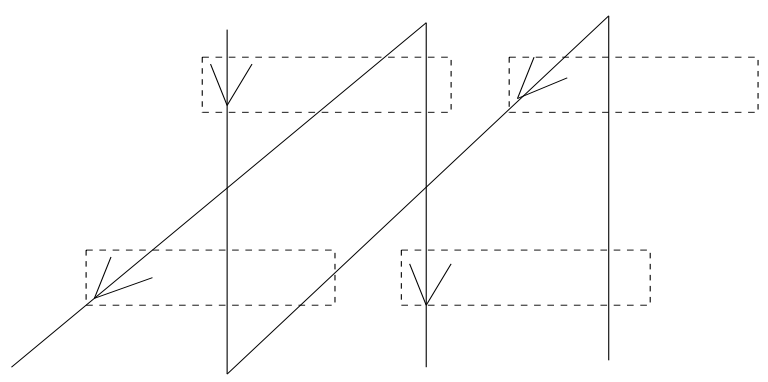

Figura 3.11: Situação de colisão para flechas de tamanho $s=3$

$\sum_{i, j=1 ; i \neq j}^{2}\left(\Lambda_{2} \phi_{2}^{i} \phi_{1}^{j} e^{i k_{j}}-a_{1} \phi_{1}^{i} \phi_{2}^{j} e^{i\left(k_{i}+k_{j}\right)}\right) \operatorname{Tr}\left[E^{x_{1}-1} A_{k_{i}}^{s_{1}} A_{k_{j}}^{s_{2}} E^{3-s_{2}} E^{N-x_{1}-s_{1}} \Omega_{P}\right]=0$

sendo $\Lambda_{2}=\Lambda_{1}\left(k_{1}\right) \Lambda_{1}\left(k_{2}\right)$. Contudo a equação (3.19) para uma única flecha nos dá

$$
\phi_{1}^{p}=\frac{-c_{1} \phi_{2}^{p}}{b_{2}-\Lambda_{1}\left(k_{p}\right)}, \quad p=1,2
$$

Inserindo esta última relação na equação (3.39) obtemos depois de algumas simplificações

$$
\begin{aligned}
& \sum_{\substack{i, j=1 ; i \neq j \\
2}} \frac{\phi_{2}^{j} \phi_{1}^{i}}{b_{2}-\Lambda_{1}\left(k_{i}\right)}\left[\left(b_{2}-\Lambda_{i}\left(k_{i}\right)\right) \Lambda_{1}\left(k_{1}\right) \Lambda_{1}\left(k_{2}\right) e^{i k_{j}}+a_{1}\left(\Lambda_{1}\left(k_{j}\right)-b_{2}\right) e^{i\left(k_{i}+k_{j}\right)}\right] \\
& \times \operatorname{Tr}\left[E^{x_{1}-1} A_{k_{i}}^{s_{1}} A_{k_{j}}^{s_{2}} E^{3-s_{2}} E^{N-x_{1}-s_{1}} \Omega_{P}\right]=0 .
\end{aligned}
$$

Devido à simetria do produto $\Lambda_{1}\left(k_{i}\right) \Lambda_{1}\left(k_{j}\right)$ pela permutação $i \rightarrow j$ é conveniente em (3.39) usarmos $\Lambda_{2}=\Lambda_{1}\left(k_{i}\right) \Lambda_{1}\left(k_{j}\right)$ de forma a obtermos

$$
\begin{aligned}
& \sum_{i, j=1 ; i \neq j}^{2} \frac{\phi_{2}^{j} \phi_{1}^{i} e^{i k_{j}}}{b_{2}-\Lambda_{1}\left(k_{i}\right)}\left[b_{2} \Lambda_{1}\left(k_{i}\right) \Lambda_{1}\left(k_{j}\right)-\Lambda_{1}\left(k_{i}\right)^{2} \Lambda_{1}\left(k_{j}\right)+a_{1} \Lambda_{1}\left(k_{j}\right) e^{i k_{i}}-a_{1} b_{2} e^{i k_{i}}\right] \\
& \times \operatorname{Tr}\left[E^{x_{1}-1} A_{k_{i}}^{s_{1}} A_{k_{j}}^{s_{2}} E^{3-s_{2}} E^{N-x_{1}-s_{1}} \Omega_{P}\right]=0 .
\end{aligned}
$$


Uma vez que $\Lambda_{1}\left(k_{i}\right)$ satisfaz

$$
-\Lambda_{1}\left(k_{i}\right)^{2}+\Lambda_{1}\left(k_{i}\right) b_{2}=-\Lambda_{1}\left(k_{i}\right) b_{1} e^{i k_{i}}+e^{i k_{i}}\left(b_{2} b_{1}-c_{2} c_{1}\right), \quad i=1,2,
$$

a equação (3.42) pode ser reescrita como

$$
\begin{aligned}
& \sum_{i, j=1 ; i \neq j}^{2} \frac{\phi_{2}^{j} \phi_{1}^{i} e^{i\left(k_{j}+k_{i}\right)}}{b_{2}-\Lambda_{1}\left(k_{i}\right)}\left[-b_{1} \Lambda_{1}\left(k_{i}\right) \Lambda_{1}\left(k_{j}\right)+\Lambda_{1}\left(k_{j}\right)\left(b_{2} b_{1}-c_{2} c_{1}\right)+a_{1}\left(\Lambda_{1}\left(k_{j}\right)-a_{1} b_{2}\right]\right. \\
& \times \operatorname{Tr}\left[E^{x_{1}-1} A_{k_{i}}^{s_{1}} A_{k_{j}}^{s_{2}} E^{3-s_{2}} E^{N-x_{1}-s_{1}} \Omega_{P}\right]=0 .
\end{aligned}
$$

Para que esta última relação seja satisfeita para quaisquer valores de $x_{1}$ devemos impor que $\operatorname{Tr}\left[E^{x_{1}-1} A_{k_{i}}^{s_{1}} A_{k_{j}}^{s_{2}} E^{3-s_{2}} E^{N-x_{1}-s_{1}} \Omega_{P}\right] \neq 0$, o que nos dá

$$
\begin{aligned}
& A_{k_{1}}^{s_{1}} A_{k_{2}}^{s_{2}} \frac{\phi_{2}^{1} \phi_{1}^{2}}{\phi_{2}^{1} \phi_{1}^{1}} \frac{b_{2}-\Lambda_{1}\left(k_{2}\right)}{b_{2}-\Lambda_{1}\left(k_{1}\right)}= \\
& -\frac{\left(-\Lambda_{1}\left(k_{2}\right) \Lambda_{1}\left(k_{1}\right) b_{1}+\Lambda_{1}\left(k_{1}\right)\left(b_{2} b_{1}-c_{2} c_{1}+a_{1}\right)-a_{1} b_{2}\right)}{\left(-\Lambda_{1}\left(k_{2}\right) \Lambda_{1}\left(k_{1}\right) b_{1}+\Lambda_{1}\left(k_{2}\right)\left(b_{2} b_{1}-c_{2} c_{1}+a_{1}\right)-a_{1} b_{2}\right)} A_{k_{2}}^{s_{1}} A_{k_{1}}^{s_{2}} .
\end{aligned}
$$

Entretanto de (3.40) temos que

$$
\frac{\phi_{2}^{1} \phi_{1}^{2}}{\phi_{2}^{2} \phi_{1}^{1}}=\frac{b_{2}-\Lambda_{1}\left(k_{1}\right)}{b_{2}-\Lambda_{1}\left(k_{2}\right)}
$$

Deste modo, para que o nosso ansatz funcione, as matrizes $A_{k_{1}}^{s_{l}}$ e $A_{k_{2}}^{s_{m}}, l, m=(1,2)$ deverão satisfazer as seguintes relações de comutação.

$$
A_{k_{j}}^{s_{l}} A_{k_{i}}^{s_{m}}=s\left(k_{j}, k_{i}\right) A_{k_{i}}^{s_{l}} A_{k_{j}}^{s_{m}}, \quad A_{k_{i}}^{s_{l}} A_{k_{i}}^{s_{m}}=0, \quad(i=1,2), \quad(l, m=1,2),
$$

sendo

$$
s\left(k_{j}, k_{i}\right)=-\frac{\Lambda_{1}\left(k_{i}\right) \Lambda_{1}\left(k_{j}\right) b_{1}-\Lambda_{1}\left(k_{j}\right)\left(b_{2} b_{1}-c_{2} c_{1}+a_{1}\right)+a_{1} b_{2}}{\Lambda_{1}\left(k_{i}\right) \Lambda_{1}\left(k_{j}\right) b_{1}-\Lambda_{1}\left(k_{i}\right)\left(b_{2} b_{1}-c_{2} c_{1}+a_{1}\right)+a_{1} b_{2}} .
$$


Os parâmetros espectrais $k_{1}$ e $k_{2}$ são fixados pela propriedade ciclíca do traço dos produtos matriciais que definem nosso ansatz. Usando (3.28),(3.8) e (3.47) obtemos

$$
\begin{aligned}
& \operatorname{Tr}\left[A_{k_{l}}^{s_{1}} A_{k_{j}}^{s_{2}} E^{N-s_{1}-s_{2}+2} \Omega_{P}\right]= \\
& e^{-i k_{j} N} e^{i k_{j}\left(s_{1}+s_{2}-2\right)} e^{-i P\left(s_{2}-1\right)} s\left(k_{j}, k_{l}\right) \operatorname{Tr}\left[A_{k_{l}}^{s_{2}} A_{k_{j}}^{s_{1}} E^{N-s_{1}-s_{2}+2} \Omega_{P}\right] .
\end{aligned}
$$

Percebemos que o traço do lado esquerdo e do lado direito são diferentes se $s_{1} \neq s_{2}$. No entanto se repetirmos mais uma vez as operações realizadas para chegar na última expressão, obteremos a igualdade do traço do lado esquerdo e do lado direito, e desta forma extrairemos que

$$
\left[e^{-i k_{j} N} e^{i k_{j}\left(s_{1}+s_{2}-2\right)} s\left(k_{j}, k_{l}\right)\right]^{2} e^{-i P\left(s_{1}+s_{2}-2\right)}=1 .
$$

Uma vez que $P=k_{1}+k_{2}$, a última expressão é escrita como

$$
e^{i k_{j} N}=e^{i \frac{2 \pi m}{2}}\left(\frac{e^{i k_{j}}}{e^{i k_{l}}}\right)^{<s>-1} s\left(k_{j}, k_{l}\right), \quad m=0,1 ; \quad j \neq l=1,2 ; \quad s_{1} \neq s_{2}
$$

sendo

$$
<s>=\frac{s_{1}+s_{2}}{2} .
$$

o tamanho médio das flechas.

- Setor com $n$ flechas

Neste caso temos uma distribuição geral de flechas com domínio de interação $\left\{s_{1}, s_{2}\right.$, $\left.\ldots, s_{n}\right\}$ e a correspendente autofunção dada por (3.4) e (3.6). A equação de autovalor proveniente da aplicação da matriz de transferência nas componentes $\mid x_{1}, \alpha_{1} ; x_{2}, \alpha_{2}$; 
$\ldots ; x_{n}, \alpha_{n}>$ onde todas as flechas não estão em posições de colisão implica na identificação

$$
A_{s_{j}}^{(\alpha)}=\sum_{l=1}^{n} \phi_{\alpha}^{l} A_{k_{l}}^{s_{j}} E^{2-s_{j}}, \alpha=1,2
$$

sendo que $\left\{A_{k_{l}}^{s_{j}}\right\}, E$ e $\Omega_{P}$ satisfazem

$$
E A_{k_{l}}^{s_{j}}=e^{i k_{l}} A_{k_{l}}^{s_{j}} E, \quad A_{k_{l}}^{s_{j}} \Omega_{P}=e^{-i P\left(1-s_{j}\right)} \Omega_{P} A_{k_{l}}^{s_{j}}, \quad(l, j=1, \ldots, n) .
$$

Mais ainda, os autovalores $\Lambda_{n}$ são dados pelo produto dos autovalores do caso de uma flecha, isto é,

$$
\Lambda_{n}\left(k_{1}, k_{2}, \ldots, k_{n}\right)=\Lambda_{1}\left(k_{1}\right) \Lambda_{1}\left(k_{2}\right) \cdots \Lambda_{1}\left(k_{n}\right),
$$

sendo $\Lambda_{1}\left(k_{j}\right)$ dado por (3.20). Como antes, o momento neste setor em termos da variáveis $\left\{k_{j}\right\}$ nos fornece a relação

$$
P=\sum_{l=1}^{n} k_{l}
$$

As equações obtidas na situação em que duas flechas estão juntas (colisão) são idênticas às equações $(3.47)$

$$
\begin{aligned}
& A_{k_{j}}^{s_{t}} A_{k_{l}}^{s_{u}}=s\left(k_{j}, k_{l}\right) A_{k_{l}}^{s_{t}} A_{k_{j}}^{s_{u}}, \quad A_{k_{j}}^{s_{t}} A_{k_{j}}^{s_{u}}=0 \\
& s\left(k_{j}, k_{l}\right)=-\frac{\Lambda_{1}\left(k_{l}\right) \Lambda_{1}\left(k_{j}\right) b_{1}-\Lambda_{1}\left(k_{j}\right)\left(b_{2} b_{1}-c_{2} c_{1}+a_{1}\right)+a_{1} b_{2}}{\Lambda_{1}\left(k_{l}\right) \Lambda_{1}\left(k_{j}\right) b_{1}-\Lambda_{1}\left(k_{l}\right)\left(b_{2} b_{1}-c_{2} c_{1}+a_{1}\right)+a_{1} b_{2}}
\end{aligned}
$$

A propriedade cíclica do traço, juntamente com (3.54) e com (3.57) nos garante para cada $k_{j}$ a relação 


$$
\begin{aligned}
& \operatorname{Tr}\left[A_{k_{1}}^{s_{1}} A_{k_{2}}^{s_{2}} \cdots A_{k_{j-1}}^{s_{j}-1} A_{k_{j}}^{s_{j}} \cdots A_{k_{n}}^{s_{n}} E^{N-\sum_{i=1}^{n}\left(s_{i}-1\right)} \Omega_{P}\right]=e^{-i k_{j} N} e^{i k_{j} \sum_{i=1}^{n}\left(s_{i}-1\right)} \\
& \times e^{-i P\left(s_{j}-1\right)} \prod_{l=1}^{n} s\left(k_{j}, k_{l}\right) \operatorname{Tr}\left[A_{k_{1}}^{s_{n}} A_{k_{2}}^{s_{1}} \cdots A_{k_{j}}^{s_{j}-1} A_{k_{j+1}}^{s_{j}} \cdots A_{k_{n}}^{s_{n}-1} E^{N-\sum_{i=1}^{n}\left(s_{i}-1\right)} \Omega_{P}\right] .
\end{aligned}
$$

Similarmente ao que aconteceu no caso de duas flechas de domínios de interação distintos, o traço de ambos os lados da última equação não é o mesmo, isto é, $\left\{s_{1}, s_{2}, \ldots, s_{n}\right\} \neq\left\{s_{n}, s_{1}, \ldots, s_{n-1}\right\}$. Mas podemos através da $r$ trocas de ordem entre as matrizes $A_{k_{l}}^{s_{j}}$ (usando-se as relações de comutação) alcançar a distribuição ordenada original. Aqui $r$ é o mínimo número de rotações cíclicas de $\left\{s_{1}, s_{2}, \ldots, s_{n}\right\}$ que faz com que a configuração se repita. Neste caso obtemos

$$
\left[e^{-i k_{j} N} e^{i k_{j} \sum_{i=1}^{n}\left(s_{i}-1\right)} \prod_{j \neq l=1}^{n} s\left(k_{j}, k_{l}\right)\right]^{r} e^{-i P \frac{r}{n} \sum_{i=1}^{n}\left(s_{i}-1\right)}=1
$$

Desde que $P=\sum_{j}^{n} k_{j}$, podemos escrever esta última expressão como

$$
e^{i k_{j} N}=-e^{i \frac{2 \pi m}{r}} \prod_{l=1}^{n} s\left(k_{j}, k_{l}\right)\left(\frac{e^{i k_{j}}}{e^{i k_{l}}}\right)^{<s>-1}, \quad m=0,1, \ldots, r-1, \quad j, l=1, \ldots, n,(
$$

onde

$$
<s>=\frac{1}{n} \sum_{i=1}^{n} s_{i}
$$

é o tamanho médio das flechas. Podemos extrair dois limites interessantes deste problema. O primeiro consiste em fazer $s_{1}=s_{2}=\cdots=s_{n}=s$, ou seja, todas as flechas com o mesmo tamanho. As equações que fixam os parâmetros espectrais do ansatz matricial são escritas agora da seguinte forma

$$
e^{i k_{j} N}=-\prod_{l=1}^{n} s\left(k_{j}, k_{l}\right)\left(\frac{e^{i k_{j}}}{e^{i k_{l}}}\right)^{s-1}, \quad j, l=1, \ldots, n .
$$


Uma outra situação a corresponde fazer $s_{1}=s_{2}=\cdots=s_{n}=1$. As equações que fixam os parâmetros espectrais são escritas agora como

$$
e^{i k_{j} N}=-\prod_{l=1}^{n} s\left(k_{j}, k_{l}\right) \quad j, l=1, \ldots, n
$$

que são idênticas às equações que determinam o espectro obtido mediante a aplicação do tradicional ansatz de Bethe para o modelo de 6 vértices na rede diagonal. Resultado este, obtido por [18] usando o ansatz de Bethe de coordenadas.

\subsection{Estrutura dos autovalores da matriz de transferência do modelo de seis vértices.}

\subsubsection{Soluções Numéricas para cadeias finitas}

Restringir-nos-emos nesta seção à análise das soluções numéricas do modelo de 6 vértices usual $(s=1)$ simétrico $\left(a_{0}=a_{1}=1, b_{1}=b_{2}=b, c_{1}=c_{2}=c\right)$. Conforme mostramos na seção anterior a estrutura dos autovalores em um setor com $n$ flechas é dada por

$$
\Lambda_{n}^{l_{1}, l_{2}, \cdots, l_{n}}=\Lambda_{1}^{l_{1}}\left(k_{1}\right) \Lambda_{1}^{l_{2}}\left(k_{2}\right) \cdots \Lambda_{1}^{l_{n}}\left(k_{n}\right),
$$

com

$$
\begin{aligned}
& \Lambda_{1}^{l_{j}}\left(k_{j}\right)=\frac{b\left(1+e^{i k_{j}}\right)+l_{j}\left[b^{2}\left(1+e^{i k_{j}}\right)^{2}-4\left(b^{2}-c^{2}\right) e^{i k_{j}}\right]^{\frac{1}{2}}}{2}, \\
& l_{j}=+,-, \quad j=1, \ldots, n
\end{aligned}
$$

e as variáveis $\left\{k_{j}\right\}$ sendo soluções do sistema de equações 


$$
e^{i k_{j} N}=(-1)^{n+1} \prod_{l=1}^{n} \frac{\Lambda\left(k_{l}\right) \Lambda\left(k_{j}\right)-2 \Delta \Lambda\left(k_{j}\right)+1}{\Lambda\left(k_{l}\right) \Lambda\left(k_{j}\right)-2 \Delta \Lambda\left(k_{l}\right)+1}, \quad j=1, \ldots, n,
$$

onde definimos o parâmetro

$$
\Delta=\frac{b^{2}-c^{2}+1}{2 b}
$$

Em nosso trabalho estudamos estas equações no regime $-1<\Delta<1$, pois para estas anisotropias o modelo apresenta uma linha crítica, ou seja, os expoentes críticos variam continuamente. Nosso ponto de partida se deu em $\Delta=0$, devido à forma simplificada das equações

$$
e^{i k_{j} N}=(-1)^{n+1}, \quad j=1, \ldots, n
$$

cujas soluções são dadas por

$$
k_{j} \in\left\{-\frac{\pi(n-1)}{N},-\frac{\pi(n-3)}{N}, \ldots, \frac{\pi(n-3)}{N}, \frac{\pi(n-1)}{N}\right\}
$$

Truong e Schotte [18] baseando-se na parametrização usual do modelo de 6 vértices na sua região antiferroelétrica [3]

$$
\begin{gathered}
a(\lambda, \sigma)=\sinh (\lambda-\sigma) \quad ; \quad b(\sigma)=\sinh (\sigma) ; \quad c=\sinh (\lambda) \\
\Delta=-\cosh (\lambda), \lambda>0,
\end{gathered}
$$

introduziu uma transformação de variáveis $k_{i} \rightarrow \sigma_{i}$, sendo as novas variáveis dadas por

$$
\Lambda_{j}=\Lambda\left(\sigma_{j}\right)=\frac{a\left(\sigma_{j}\right)}{b\left(\sigma_{j}\right)}=\frac{\sinh \left(\lambda-\sigma_{j}\right)}{\sinh \left(\sigma_{j}\right)}, j=1, \ldots, n
$$


A constante de estrutura $s\left(k_{j}, k_{l}\right)$ nessas novas variáveis toma a forma simplificada

$$
s\left(k_{j}, k_{l}\right)=\frac{\sinh \left(\sigma_{l}-\sigma_{j}-\lambda\right)}{\sinh \left(\sigma_{l}-\sigma_{j}+\lambda\right)}
$$

Uma vez que $e^{i k_{j}}$ pode ser escrito como função de $\Lambda_{j}$, obtemos em termos da variável $\sigma_{j}$

$$
\begin{aligned}
e^{i k_{j}} & =\frac{\Lambda_{j}\left(\Lambda_{j}-\frac{b}{a}\right)}{\frac{b}{a}\left(\Lambda_{j}-\frac{b}{a}\right)+\frac{c^{2}}{a^{2}}}= \\
\frac{a\left(\sigma_{j}\right) a\left(\sigma+\sigma_{j}\right)}{b\left(\sigma_{j}\right) b\left(\sigma+\sigma_{j}\right)} & =\frac{\sinh \left(\lambda-\sigma_{j}\right) \sinh \left(\lambda-\sigma-\sigma_{j}\right)}{\sinh \left(\sigma_{j}\right) \sinh \left(\sigma+\sigma_{j}\right)} .
\end{aligned}
$$

Deste modo as equações que fixam o espectro tornam-se ${ }^{3}$

$$
\left[\frac{\sinh \left(\lambda-\sigma_{j}\right) \sinh \left(\lambda-\sigma-\sigma_{j}\right)}{\sinh \left(\sigma_{j}\right) \sinh \left(\sigma+\sigma_{j}\right)}\right]^{N}=-\prod_{l=1}^{n} \frac{\sinh \left(\sigma_{j}-\sigma_{l}-\lambda\right)}{\sinh \left(\sigma_{j}-\sigma_{l}+\lambda\right)}
$$

Para alcançarmos o regime de interesse $-1<\Delta<1$, que corresponde à região desordenada, é necessário fazermos as seguintes mudanças: $\lambda \rightarrow i \lambda, \sigma \rightarrow i \sigma$. Escolhendo-se $a=1$ teremos ao invés de (3.69), (3.70), (3.72) e (3.73) as seguintes equações.

$$
\begin{gathered}
a=1 ; \quad b=\frac{\sin (\sigma)}{\sin (\lambda-\sigma)}, \quad c=\frac{\sin (\lambda)}{\sin (\lambda-\sigma)}, \\
\Lambda_{j}=\frac{\sinh \left(i \lambda-\sigma_{j}\right)}{\sinh \left(\sigma_{j}\right)}, \\
e^{i k_{j}}=\frac{\sinh \left(i \lambda-\sigma_{j}\right) \sinh \left(i \lambda-i \sigma-\sigma_{j}\right)}{\sinh \left(\sigma_{j}\right) \sinh \left(i \sigma+\sigma_{j}\right)}, \quad(j=l, \ldots n),
\end{gathered}
$$

\footnotetext{
${ }^{3}$ Como tais equações fixam o espectro tanto na solução no ansatz de produto de matrizes como no ansatz de Bethe denominamos as mesmas por equações do ansatz de Bethe, como são conhecidas na literatura.
} 


$$
\left[\frac{\sinh \left(i \lambda-\sigma_{j}\right) \sinh \left(i \lambda-i \sigma-\sigma_{j}\right)}{\sinh \left(\sigma_{j}\right) \sinh \left(i \lambda+\sigma_{j}\right)}\right]^{N}=-\prod_{l=1}^{n} \frac{\sinh \left(\sigma_{j}-\sigma_{l}-i \lambda\right)}{\sinh \left(\sigma_{j}+\sigma_{l}-i \lambda\right)}
$$

sendo $j=1, \ldots, n$. Estas parametrizações são convenientes para o cálculo computacional e analítico conforme mostraremos na próxima seção. A transformação de coordenada $\left\{k_{j}\right\} \rightarrow\left\{\sigma_{j}\right\}$ nos fornece para cada $j=1, \ldots, n$ dois possíveis valores para a variável $\sigma_{j}$, isto é,

$$
\begin{aligned}
& y_{j}^{l_{j}}=e^{2 \sigma_{j}^{l_{j}}}= \\
& \frac{\left(e^{i k_{j}}-1\right) \cos (\sigma)+l_{j}\left[\left(e^{i k_{j}}-1\right)^{2} \cos ^{2}(\sigma)-\left(e^{i k_{j}}-e^{-2 i \lambda}\right)^{2}\right]^{\frac{1}{2}}}{2 e^{i \sigma}\left(e^{i k_{j}}-e^{-i 2 \lambda}\right)}
\end{aligned}
$$

com $j=1, \ldots, n$. Escrevendo $\sigma_{j}^{l_{j}}=\sigma_{j}^{l_{j}, R}+i \sigma_{j}^{l_{j}, I}$, obtemos

$$
\sigma_{j}^{l_{j}, R}=\frac{\arg \left(y_{j}^{l_{j}}\right)}{2}, \quad \sigma_{j}^{l_{j}, I}=-\frac{\ln \left(\left|y_{j}^{l_{j}}\right|\right)}{2} .
$$

Em termos da variável $\sigma_{j}^{l_{j}}$, os autovalores do setor da matriz de transferência associados às configurações de $n$ flechas, podem ser escritos como

$$
\begin{aligned}
& \Lambda_{n}^{l_{1}, l_{2}, \ldots, l_{n}}=<l_{n}\left|\otimes \cdots<l_{2}\right| \otimes<l_{1} \mid\left(\begin{array}{cc}
\Lambda_{1}^{+}\left(\sigma_{1}^{+}\right) & 0 \\
0 & \Lambda_{1}^{-}\left(\sigma_{1}^{-}\right)
\end{array}\right) \otimes\left(\begin{array}{cc}
\Lambda_{1}^{+}\left(\sigma_{2}^{+}\right) & 0 \\
0 & \Lambda_{1}^{-}\left(\sigma_{2}^{-}\right)
\end{array}\right) \\
& \otimes \cdots \otimes\left(\begin{array}{cc}
\Lambda_{1}^{+}\left(\sigma_{n}^{+}\right) & 0 \\
0 & \Lambda_{1}^{-}\left(\sigma_{n}^{-}\right)
\end{array}\right)\left|l_{1}>\otimes\right| l_{2}>\ldots \otimes \mid l_{n}>,
\end{aligned}
$$

onde

$$
\mid l_{j}>=\left(\begin{array}{l}
\delta_{l_{j},+} \\
\delta_{l_{j},-}
\end{array}\right)
$$


sendo

$$
\Lambda_{1}^{m}\left(\sigma_{j}^{l_{j}}\right)=\frac{\sinh \left(i \lambda-\sigma_{j}^{l_{j}}\right)}{\sinh \left(\sigma_{j}^{l_{j}}\right)} \delta_{m, l_{j}}, \quad l_{j}, m=+,-, \quad(j=1, \ldots, n) .
$$

Os valores de $\sigma_{j}^{l_{j}}$ são dados pelas soluções das equações (3.77), isto é,

$$
\left[\frac{\sinh \left(i \lambda-\sigma_{j}^{l_{j}}\right) \sinh \left(i \lambda-i \sigma-\sigma_{j}^{l_{j}}\right)}{\sinh \left(\sigma_{j}^{l_{j}}\right) \sinh \left(i \lambda+\sigma_{j}^{l_{j}}\right)}\right]^{N}=-\prod_{m=1}^{n} \frac{\sinh \left(\sigma_{j}^{l_{j}}-\sigma_{m}^{l_{m}}+i \lambda\right)}{\sinh \left(\sigma_{j}^{l_{j}}-\sigma_{m}^{l_{m}}-i \lambda\right)},
$$

para $j=1,2, \ldots, n$.

Para entendermos a estrutura das raízes $\left\{\sigma_{j}\right\}$ das equações (3.83) consideremos inicialmente o caso $\Delta=0$. Neste caso as soluções em termos das variáveis $\left\{k_{j}\right\}$ são dados por (3.68). A equação (3.78) nos diz que as raízes $\left\{\sigma_{j}^{l_{j}}\right\}$ são números complexos cuja parte imaginária assume apenas dois possíveis valores

$$
\begin{aligned}
& \sigma_{j}^{+, I}=\frac{\left(\frac{\pi}{2}-\sigma\right)}{2}, \\
& \sigma_{j}^{-, I}=\frac{\left(\frac{\pi}{2}+\sigma\right)}{2} .
\end{aligned}
$$

Para compreender a estrutura das raízes de (3.83) para $\Delta \neq 0\left(\lambda \neq \frac{\pi}{2}\right)$ e para diferentes valores de $\sigma, n$ e $N$, tivemos que buscar soluções numéricas das equações (3.83). A diagonalização direta da matriz de transferência (força bruta ${ }^{4}$ ), para cadeias de tamanho maxímo $N=6$, nos permitiu associar certas configurações de raízes aos maiores autovalores dos diversos setores. Através destes dois caminhos conseguimos mapear algumas soluções das equações do ansatz de Bethe. Conseguimos também, através da diagonalização direta, observar as seguintes características gerais dos autovalores em um dado setor com $n$ flechas:

\footnotetext{
${ }^{4}$ Por força bruta ou método direto mencionamos a diagonalização numérica de todos os autovalores em um dado setor da matriz de transferência
} 
(1)- Os autovalores são números complexos em geral.

(2)- O autovalor que possui a maior parte real é um autovalor real puro.

(3)- Os autovalores complexos surgem aos pares conjugados (traço da matriz de transferência deve ser real).

Com base nos resultados da diagonalização numérica de $T_{D-D}$ e no estudo analítico $(\Delta=0)$ e numérico $(\Delta \neq 0)$ das equações do ansatz de Bethe (3.83) pudemos com grande confiança formular as seguintes hipóteses para as distribuições de raízes $\left\{\sigma_{j}^{l_{j}}\right\}$ : Hipótese 1: O maior autovalor em um dado setor com $n$ flechas possui uma distribuição de raízes com parte real simétrica e parte imaginária fixa dada por $\frac{\lambda-\sigma}{2}$. Hipótese 2: A energia livre no limite termodinâmico é caracterizada por um autovalor residente no setor de $N$ flechas.

Tais distribuições simétricas implicam que o auto-estado correspodente possui momento nulo.

\subsubsection{Estrutura dos estados "excitados"}

A obtenção das soluções numéricas das equações não lineares de (3.83) é algo não simples e infelizmente não existe método que garanta em geral a obtenção de tais soluções. O método numérico usual em tais casos é o método de Newton e a convergência do mesmo além de depender do "chute" inicial, depende fortemente da parametrização escolhida para expressar as incógnitas do sistema. Em nossa análise numérica diversas soluções foram obtidas. A seguir discutiremos algumas destas soluções.

-Segundo maior autovalor real (estado marginal) ${ }^{5}$

Setor com $n=N$ flechas

\footnotetext{
${ }^{5}$ Esta nomenclatura antecipa a identificação deste estado com o operador marginal do modelo, conforme veremos mais adiante.
} 
Na variável $\sigma_{j}$ observamos numericamente que para $\lambda$ e $\sigma$ tais que, $\frac{\pi}{3}<\lambda \leq \frac{\pi}{2}$, e $-\lambda<$ $\sigma<\lambda$, o segundo maior autovalor real no setor com $N$ flechas é caracterizado por um conjunto $\left\{\sigma_{j}^{R}\right\}\left(\sigma_{1}^{R}<\sigma_{2}^{R}<\cdots<\sigma_{N}^{R}\right)$, similar ao conjunto do maior autovalor real localizado neste setor (para $\Delta=0$, essa distribuição é a mesma do maior autovalor real). Entretanto o correspondente conjunto $\left\{\sigma_{j}^{I}\right\} \equiv\left(\sigma_{1}^{I}, \sigma_{2}^{I}, \ldots, \sigma_{N}^{I}\right)$ deste autovalor é tal que $\sigma_{1}^{I}=\sigma_{N}^{I}=\frac{(\lambda-\sigma-\pi)}{2}$, enquanto que $\sigma_{2}^{I}=\cdots=\sigma_{N-1}^{I}=\frac{(\lambda-\sigma)}{2}$. Na figura $3.12 \mathrm{~b}$ representamos este estado para uma rede de tamanho $N=6 \operatorname{com} n=6$ flechas.

\section{Setor com $n \neq N$ flechas}

O estado marginal neste setor é caracterizado por raízes $\left\{\sigma_{j}^{l_{j}}\right\}$ com a mesma parte imaginária constante $\sigma_{j}^{I}=\frac{\lambda-\sigma}{2}$, enquanto que a parte real é construída de forma que o maior $\sigma_{j}^{+, R}$ e menor $\sigma_{j}^{-, R}$ não esteja tão concentrado, quando comparado com o maior autovalor deste setor.

•Estados de momento não nulo

Neste caso a nossa análise se restringirá apenas no caso especial em que $\Delta=0$. Para construirmos estados de momento não nulo, devemos escolher um conjunto de $\left\{k_{j}\right\}$ tal que a soma seja não nula. Como $\Lambda^{l_{j}}\left(k_{j}\right)$ possui dois valores distintos para o mesmo $k_{j}\left(\Lambda^{+}\left(k_{j}\right) \neq \Lambda^{-}\left(k_{j}\right)\right)$ podemos construir um estado de momento, a partir de um conjunto $\left\{k_{j}\right\}$ contendo números de ondas degenerados. Em virtude dessa degenerescência da variável $k_{j}$, um dos possíveis estados de momento $p_{1}=-\frac{2 \pi}{N}$, ou $p_{2}=\frac{2 \pi}{N}$ que podemos construir em uma rede de tamanho $N$ possuem autovalores dados pela seguinte configuração

$$
\begin{aligned}
& \Lambda_{n}^{p_{1}}=\Lambda_{1}^{+}\left(-k_{\max }\right) \cdots \Lambda_{1}^{-}\left(-k_{\min }\right) \Lambda_{1}^{+}\left(-k_{\min }\right) \cdots \Lambda_{1}^{+}\left(k_{\max }\right), \\
& \Lambda_{n}^{p_{2}}=\Lambda_{1}^{+}\left(-k_{\max }\right) \cdots \Lambda_{1}^{-}\left(k_{\min }\right) \Lambda_{1}^{+}\left(k_{\min }\right) \cdots \Lambda_{1}^{+}\left(k_{\max }\right),
\end{aligned}
$$

com $k_{\text {max }}$ e $k_{\text {min }}$ dados por 


$$
\begin{aligned}
& k_{\max }=\frac{\pi(N-1)}{N}, \\
& k_{\operatorname{mim}}=\frac{\pi}{N} .
\end{aligned}
$$

Na variável $\sigma_{j}$, o estado acima mencionado com momento $p_{1}$ é construído através da inversão da parte imaginária $\frac{i(\lambda-\sigma)}{2}$ para $-\frac{i \pi}{2}+\frac{i(\lambda-\sigma)}{2}$ da raíz com a maior parte real negativa $\sigma_{\min }^{-, R}$ do estado de maior autovalor no setor. Analogamente o estado com momento $p_{2}$ é construído através da inversão da parte imaginária $\frac{i(\lambda-\sigma)}{2}$ para $\frac{-i \pi}{2}+$ $\frac{i(\lambda-\sigma)}{2}$ da raiz com a menor parte real positiva $\sigma_{\min }^{+, R}$ do estado de maior autovalor no setor. Nas figuras 3.12c e 3.12d mostramos estes estados para uma rede de tamanho $N=6 \operatorname{com} n=6$ flechas.

\subsubsection{Observações finais}

Os autovalores de valor máximo em um dado setor com $n$ flechas puderam ser calculados (computacionalmente) para diversos valores da anisotropia $-1<\Delta<1$ resolvendo-se as equações do ansatz de Bethe (3.83). Os demais estados não puderam ser verificados no mesmo domínio com que verificamos o maior autovalor devido às instabilidades numéricas nas equações. Não conseguimos também compreender a estrutura dos estados de momento não nulo em $\Delta \neq 0$. Por falta de tempo, não pudemos encontrar estados com configurações de raízes do tipo cordas ("strings") análogas àquelas que surgem nas equações do ansatz de Bethe relacionados com o modelo XXZ [19]. Estes estados são caracterizados por divergências nos denominadores do lado direito e do lado esquerdo das equações do ansatz de Bethe (3.83). 


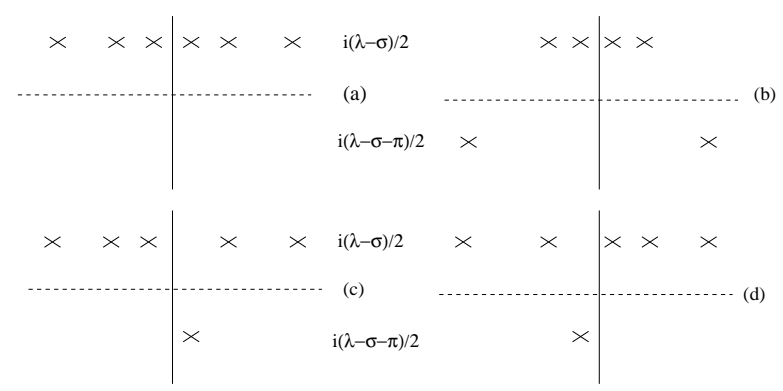

Figura 3.12: Configuração das raízes da equação de ansatz de Bethe para $N=n=6$ :(a) configuração do estado de maior autovalor; (b) configuração do estado marginal; (c) e (d) configuração correspondente ao maior autovalor com momento $-\frac{2 \pi}{N}$ e $\frac{2 \pi}{N}$ respectivamente.

\subsection{Limite Termodinâmico}

\subsubsection{Introdução}

Abordaremos neste capítulo, um estudo do maior autovalor da matriz de transferência do modelos de 5 vértices interagentes, para o caso especial em que $a_{1}=a_{2}, b_{1}=b_{2}$, $c_{1}=c_{2}$ (caso simétrico). Apresentaremos apenas o caso especial $s_{1}=s_{2}=\ldots=s_{n}=$ 1 do modelo de 5 vértices interagentes, que corresponde ao modelo de 6 vertices ${ }^{6}$.

\subsubsection{Fases Termodinâmicas}

Conforme é bem sabido da literatura, o modelo de seis vértices simétrico apresenta três fases físicas diferentes.

Ferroelétrica: caracterizada pela região onde $a>b+c$ ou $c>a+b$.

Desordenada: caracterizada pela região onde $c<\frac{a+b+c}{2}$.

Antiferroelétrica: caracterizada pela região onde $c>a+b$.

\footnotetext{
${ }^{6}$ Embora não deva apresentar dificuldade adicional, tais cálculos para os casos gerais do modelo ainda não foram feitos
} 
Tais resultados foram obtidos pela diagonalização da matriz de transferência linhapara-linha do modelo com condições periódicas (veja por exemplo [3]). Em nosso trabalho obtivemos o espectro da matriz de transferência diagonal-para-diagonal com condições de contorno toroidais. Assim sendo, é interessante a re-obtenção das fases acima mencionadas.

\subsection{2 $\Delta>1$.}

Foi mostrado que a estrutura dos autovalores para o setor com $n$ flechas possue a forma apresentada em (3.55). A nossa idéia agora é tentar mostrar de forma não rigorosa que o maior autovalor no regime $\Delta>1$ pertence ao setor com $n=0$ flechas, ou seja, $\Lambda_{0}^{\max }>\Lambda_{n}^{\max }$ para $n=1, \ldots, 2 N$, sendo $\Lambda_{n}^{\max }$ o maior autovalor no setor com $n$ flechas.

Iniciaremos nossa demonstração comparando o autovalor $\Lambda_{0}^{\max } \operatorname{com} \Lambda_{1}^{\max }$. Como consequência do teorema de Perron-Frobenius, o maior autovalor está associado à um autovetor que possui todas entradas positivas. Os únicos autovetores no setor com uma flecha que possui todas entradas positivas são os autovetores que possuem momento nulo, ou seja, $k=0$. Substituindo-se este parâmetro em (3.20) obtemos os autovalores

$$
\begin{gathered}
\Lambda_{1}^{+}(k=0)=b+c, \\
\Lambda_{1}^{-}(k=0)=b-c .
\end{gathered}
$$

Isto nos garante que o maior autovalor no setor com uma flecha é dado por $\Lambda_{1}^{\max }=$ $b+c$, e deste modo fica demostrado que $\Lambda_{0}^{\max }>\Lambda_{1}^{\max }$, pois na região ferroelétrica temos $1>b+c, \mathrm{e}$

$$
\Lambda_{0}^{\max }=1, \quad \Lambda_{1}^{\max }=b+c .
$$


Para fornecer um argumento que $\Lambda_{0}^{\max }>\Lambda_{n}^{\max }$, para $n>1$, imaginemos inicialmente que exista uma combinação especial de $\left\{k_{1}, \ldots, k_{n}\right\}$ que seja solução das equações do ansatz de Bethe no setor que possui $n$ flechas e que produz o maior autovalor. Mais especificamente, o maior autovalor associado a estes números de onda deve possuir a maior parte real entre aqueles autovalores pertencentes a este específico setor com $n$ flechas. Evidentemente a obteção desse conjunto especial $\left\{k_{1}, \ldots, k_{n}\right\}$ para $\Delta \neq 0$ não é um problema trivial do ponto de vista analítico. Entretanto é possível provar a existência de um limite superior $\Lambda_{n}^{\mathrm{MAX}}$ para $\Lambda_{n}^{\max }$, isto é, $\Lambda_{n}^{\mathrm{MAX}}>\Lambda_{n}^{\max }$. Tal autovalor hipotético é obtido relachando-se por completo a regra do gelo, isto é,

$$
\Lambda_{n}^{\mathrm{MAX}}=\left(\Lambda_{1}^{\max }\right)^{n}=(b+c)^{n}<1 .
$$

Uma vez que $1>b+c$ (região ferroelétrica), concluímos que $\Lambda_{0}^{\max }>\Lambda_{n}^{\max }$.

\subsection{3 $-1<\Delta<1$}

O cálculo do maior autovalor de $T_{D-D}$ no regime $-1<\Delta<1$ é feito explorando-se as hipóteses da seção (3.4.1). Antes de iniciarmos nosso cálculo mostraremos que a observação enunciada na hipótese 1 sobre a distribuição de raízes $\left\{\sigma_{j}\right\}(j=1, \ldots, n)$ para o maior autovalor de uma dado setor é equivalente a dizer que as raízes $\left\{k_{j}\right\}$ correspondentes a tal distribuição são reais. Escrevendo

$$
\sigma_{j}=\sigma_{j}^{R}+i \sigma_{j}^{I}, \quad \operatorname{com} \sigma_{j}^{I}=f(\lambda, \sigma),
$$

a variável $k_{j}$ correspondente é dada por

$$
e^{i k_{j}}=\frac{\sinh \left[i(\lambda-f(\lambda, \sigma))-\sigma_{j}^{R}\right]}{\sinh \left[\sigma_{j}^{R}+i f(\lambda, \sigma)\right]} \frac{\sinh \left[-\sigma_{j}^{R}+i(\lambda-\sigma-f(\lambda, \sigma))\right]}{\sinh \left[\sigma_{j}^{R}+i(\sigma+f(\lambda, \sigma))\right]} .
$$


Caso a variável $k_{j}$ seja real esta última expressão deve ser unimodular, o que é possível caso

$$
f(\lambda, \sigma)=\frac{\lambda-\sigma}{2} .
$$

Assim a distribuição de raízes referente ao estado de maior autovalor, por ser o mesmo de momento nulo (teorema de Perron-Frobenius) é dada pela distribuição simétrica de raízes

$$
\sigma_{j}=\sigma_{j}^{R}+i \frac{\lambda-\sigma}{2},
$$

Substituindo esta última expressão em (3.91) obtemos

$$
\frac{\sinh \left[\sigma_{j}^{R}-i g(\lambda, \sigma)\right]}{\sinh \left[\sigma_{j}^{R}+i g(\lambda, \sigma)\right]} \frac{\sinh \left[\sigma_{j}^{R}-i f(\lambda, \sigma)\right]}{\sinh \left[\sigma_{j}^{R}+i f(\lambda, \sigma)\right]} .
$$

Consequentemente as equações do ansatz de Bethe nestas novas variáveis são escritas como

$$
\left[\frac{\sinh \left[\sigma_{j}^{R}-i g(\lambda, \sigma)\right]}{\sinh \left[\sigma_{j}^{R}+i g(\lambda, \sigma)\right]} \frac{\sinh \left[\sigma_{j}^{R}-i f(\lambda, \sigma)\right]}{\sinh \left[\sigma_{j}^{R}+i f(\lambda, \sigma)\right]}\right]^{N}=-\prod_{l=1}^{n} \frac{\sinh \left(\sigma_{j}^{R}-\sigma_{l}^{R}-i \lambda\right)}{\sinh \left(\sigma_{j}^{R}-\sigma_{l}^{R}+i \lambda\right)}
$$

onde $j=1, \ldots, n$, e

$$
g(\lambda, \sigma)=\frac{\lambda+\sigma}{2} .
$$

Para prosseguirmos é conveniente usarmos a relação

$$
\begin{aligned}
& \frac{\sinh (z-i w)}{\sinh (z+i w)}=-e^{i \theta(z, w)} \\
& \theta(z, w)=\arctan \left(\frac{2 \tanh (z) \cot (w)}{1-\tanh ^{2}(z) \cot ^{2}(w)}\right),
\end{aligned}
$$


válida para $z, w$ reais. Utilizando esta úlltima expressão as equações (3.95) podem então ser escritas como

$$
e^{i N\left(\theta_{1}\left(\sigma_{j}^{R}\right)+\theta_{2}\left(\sigma_{j}^{R}\right)\right)}=(-1)^{n+1} e^{i \sum_{l=1}^{n} \theta\left(\sigma_{l}^{R}, \sigma_{j}^{R}\right)}
$$

sendo

$$
\begin{aligned}
& \left.\theta_{1}\left(\sigma_{j}^{R}\right)=\arctan \left(\frac{2 \tanh \left(\sigma_{j}^{R}\right) \cot (g(\lambda, \sigma))}{1-\tanh ^{2}\left(\sigma_{j}^{R}\right) \cot ^{2}(g(\lambda, \sigma)}\right)\right), \\
& \left.\theta_{2}\left(\sigma_{j}^{R}\right)=\arctan \left(\frac{2 \tanh \left(\sigma_{j}^{R}\right) \cot (f(\lambda, \sigma))}{1-\tanh ^{2}\left(\sigma_{j}^{R}\right) \cot ^{2}(f(\lambda, \sigma)}\right)\right), \\
& \theta_{3}\left(\sigma_{l}^{R}, \sigma_{j}^{R}\right)=\arctan \left(\frac{2 \tanh \left(\sigma_{j}^{R}-\sigma_{l}^{R}\right) \cot (\lambda)}{1-\tanh ^{2}\left(\sigma_{j}^{R}-\sigma_{l}^{R}\right) \cot ^{2}(\lambda)}\right) .
\end{aligned}
$$

Uma vez que $\theta_{1}, \theta_{2}$ e $\theta_{3}$ são funções reais, quando tomamos o logarítimo em (3.98) obtemos

$$
N\left[\theta_{1}\left(\sigma_{j}^{R}\right)+\theta_{2}\left(\sigma_{j}^{R}\right)\right]=2 \pi I_{j}+\sum_{l=1}^{n} \theta_{3}\left(\sigma_{l}^{R}, \sigma_{j}^{R}\right), \quad j=1, \ldots, n .
$$

sendo $I_{j}$ inteiros ou semi-inteiros caso $n$ seja ímpar ou par, respectivamente. Verificamos mediante a resolução numérica das equações do ansatz de Bethe que a escolha apropriada para os inteiros $\left\{I_{j}\right\}$ relacionados ao maior autovalor do setor com $n$ flechas é dada por

$$
I_{j}=j-\frac{n+1}{2}, \quad j=1, \ldots, n .
$$

Vamos obter agora a função distribuição de $\sigma_{j}^{R}$ no limite termodinâmico referente ao estado de maior autovalor em um dado setor de $n$ flechas. Para isto devemos impor que $\left\{\sigma_{1}^{R}, \ldots, \sigma_{n}^{R}\right\}$ sejam distintos e distribuídos em torno da origem em algum 
intervalo simétrico $(-Q, Q)$ (condição de momento nulo). Mais ainda, a distribuição deve ser a mais condensada possível (para termos a menor energia livre).

É conveniente definirmos a função $\rho\left(\sigma^{R}\right)$ que fornece a distribuição de $\sigma_{j}^{R}$, isto é, $2 N \rho\left(\sigma^{R}\right) d \sigma^{R}$ nos dá o número de $\sigma^{R} s$ no intervalo entre $\sigma^{R}$ e $\sigma^{R}+d \sigma^{R}$. Consequentemente $\rho\left(\sigma^{R}\right)$ deve satisfazer

$$
\int_{-Q}^{Q} \rho\left(\sigma^{R}\right) d \sigma^{R}=\frac{n}{2 N}
$$

Imaginemos que estamos considerando o nosso sistema com um número de flechas $n$ fixo. Neste caso a densidade de energia livre para um sistema finito é escrita como

$$
f=-k_{B} T \sum_{j=1}^{n} \frac{\ln \left(\Lambda_{j}\right)}{N},
$$

no limite termodinâmico assume a forma

$$
f=-2 k_{B} T \int_{-Q}^{Q} \ln \left(\Lambda^{R}\right) \rho\left(\sigma^{R}\right) d\left(\sigma^{R}\right) .
$$

Para um dado valor $\sigma^{R}$ da variável $\sigma_{j}^{R}$, o inteiro $I_{j}+\frac{n+1}{2}$ nos dá o número de $\sigma_{l}^{R} s$ com $l<j$. Deste modo a equação (3.100) se torna

$$
\begin{aligned}
& N\left[\theta_{1}\left(\sigma^{R}\right)+\theta_{2}\left(\sigma^{R}\right)\right]=-\pi(n+1) \\
& +4 \pi N \int_{-Q}^{\sigma^{R}} \rho\left(\sigma^{R^{\prime \prime}}\right) d \sigma^{R^{\prime \prime}}+2 N \int_{-Q}^{Q} \theta_{3}\left(\sigma^{R^{\prime \prime}}, \sigma^{R}\right) \rho\left(\sigma^{R^{\prime \prime}}\right) d \sigma^{R^{\prime \prime}}
\end{aligned}
$$

Diferenciando-se esta úlltima expressão com respeito a $\sigma^{R}$ e dividindo-se por $N$ obtemos

$$
\frac{d}{d \sigma^{R}}\left[\theta_{1}\left(\sigma^{R}\right)+\theta_{2}\left(\sigma^{R}\right)\right]=4 \pi \rho\left(\sigma^{R}\right)+2 \int_{-Q}^{Q} \frac{\partial}{\partial \sigma^{R}} \theta_{3}\left(\sigma^{R^{\prime \prime}}, \sigma^{R}\right) \rho\left(\sigma^{R^{\prime \prime}}\right) d \sigma^{R^{\prime \prime}}
$$


Uma vez efetuado estas derivadas encontramos a seguinte expressão para a função distribuição

$$
\begin{aligned}
\rho\left(\sigma^{R}\right)= & \frac{1}{2 \pi} \frac{\sin (\sigma+\lambda)}{\cosh \left(2 \sigma^{R}\right)-\cos (\sigma+\lambda)}+\frac{1}{2 \pi} \frac{\sin (\lambda-\sigma)}{\cosh \left(2 \sigma^{R}\right)-\cos (\lambda-\sigma)} \\
& -\frac{1}{\pi} \int_{-Q}^{Q} \frac{\sin (2 \lambda)}{\cosh \left(2\left(\sigma^{R}-\sigma^{R^{\prime \prime}}\right)\right)-\cos (2 \lambda)} \rho\left(\sigma^{R^{\prime \prime}}\right) d \sigma^{R^{\prime \prime}} .
\end{aligned}
$$

Isto nos mostra que a função distribuição $\rho$ é dada por uma equação integral. Como o integrando em (3.107) depende apenas da diferença das variáveis, podemos usar a transformada de fourier para resolver esta equação no caso especial em que $Q=\infty$.

Definindo

$$
\bar{\rho}(x)=\frac{1}{2 \pi} \int_{-\infty}^{\infty} \rho\left(\sigma^{R}\right) e^{i x \sigma^{R}} d \sigma^{R}
$$

e fazendo a transformada de fourier de (3.107) obtemos em termos da variável $x$

$$
\begin{aligned}
& 2 \pi \bar{\rho}(x)=\frac{\sinh \left[(\pi-(\sigma+\lambda)) \frac{x}{2}\right]}{2 \sinh \left(\frac{x \pi}{2}\right)} \\
& +\frac{\sinh \left[(\pi-(\lambda-\sigma)) \frac{x}{2}\right]}{2 \sinh \left(\frac{x \pi}{2}\right)}-2 \pi \frac{\sinh \left[(\pi-2 \lambda) \frac{x}{2}\right]}{\sinh \left(\frac{x \pi}{2}\right)} \bar{\rho}(x) .
\end{aligned}
$$

Simplificando esta última equação obtemos

$$
2 \pi \bar{\rho}(x)=\frac{\cosh \left(\frac{x \sigma}{2}\right) \sinh \left(\frac{x(\pi-\lambda)}{2}\right)}{\sinh \left(\frac{x \pi}{2}\right)}+\frac{2 \pi \sinh \left[\frac{(\pi-2 \lambda) x}{2}\right] \bar{\rho}(x)}{\sinh \left(\frac{x \pi}{2}\right)} .
$$

Definindo-se por conveniência a função $2 \pi \bar{\rho}(x)=\bar{\varphi}(x)$ obtemos

$$
\bar{\varphi}(x)=\frac{\cosh \left(\frac{x \sigma}{2}\right) \operatorname{sech}\left(\frac{x \lambda}{2}\right)}{2} .
$$


- Localização do setor de maior autovalor

Da úlltima expressão extraímos que $\bar{\varphi}(0)=\frac{1}{2}$. Além disso sabemos que a transformação de $\varphi\left(\sigma^{R}\right)$ para $\bar{\varphi}(x)$ é dada por

$$
\bar{\varphi}(x)=\frac{1}{2 \pi} \int_{-\pi}^{\pi} \varphi\left(\sigma^{R}\right) e^{i x \sigma^{R}} d \sigma^{R}
$$

e que a normalização para $\varphi\left(\sigma^{R}\right)$ é escrita como,

$$
\frac{1}{2 \pi} \int_{-\infty}^{\infty} \varphi\left(\sigma^{R}\right) d \sigma^{R}=\frac{n}{2 N}
$$

A partir de (3.112) e (3.113) vemos que $\bar{\varphi}(0)=\frac{1}{2}$, e deste modo o maior autovalor deve pertencer ao setor onde $n=N$, e assim sendo a hipótese 2 anunciada na seção 3.4.1 decorre da hipótese 1.

\section{-Energia livre}

Calculando a transformada inversa de fourier de $\bar{\varphi}(x)$ obtemos

$$
\varphi\left(\sigma^{R}\right)=\frac{8 \pi}{\lambda} \frac{\cosh \left(\frac{\sigma^{R} \pi}{\lambda}\right) \cos \left(\frac{\pi \sigma}{2 \lambda}\right)}{\left[\cosh \left(\frac{2 \sigma^{R} \pi}{\lambda}\right)+\cos \left(\frac{\sigma \pi}{\lambda}\right)\right]}, \quad \sigma^{R}>0
$$

A energia livre (3.103) é agora escrita como

$$
f_{\infty}=-k_{B} T \int_{0}^{\infty} \frac{\varphi\left(\sigma^{R}\right)}{\pi} \ln \left(\left|\Lambda\left(\sigma^{R}\right)\right|\right) d \sigma^{R},
$$

ou seja, 


$$
\begin{aligned}
& f_{\infty}=-k_{B} T \int_{0}^{\infty} \frac{2}{\lambda} \frac{\cosh \left(\frac{\sigma^{R} \pi}{\lambda}\right) \cos \left(\frac{\pi \sigma}{2 \lambda}\right)}{\left[\cosh \left(\frac{2 \sigma^{R} \pi}{\lambda}\right)+\cos \left(\frac{\sigma \pi}{\lambda}\right)\right]} \ln \left[\frac{\cosh \left(2 \sigma^{R}\right)-\cos (\lambda+\sigma)}{\cosh \left(2 \sigma^{R}\right)-\cos (\lambda-\sigma)}\right] d \sigma^{R} \\
& 0<\lambda<\pi, \quad-\lambda<\sigma<\lambda
\end{aligned}
$$

Na tabela I mostramos na última linha $(N \rightarrow \infty)$ o valor numérico desta integral para $\sigma=0.5$ e diversos valores de $\lambda$. Mostramos também nesta tabela resultados do cálculo do logarítimo do maior autovalor da matriz de transferência $-\frac{\ln \left(\Lambda_{\max }(N)\right)}{N} \mathrm{em}$ redes de tamanhos $N$ finito e no setor de $N$ flechas. Tais resultados foram obtidos mediante a resolução numérica das equações do ansatz de Bethe.

\section{Tabela I}

\begin{tabular}{|c|c|c|c|c|}
\hline $\mathrm{N}$ & $\lambda=\frac{2 \pi}{3}$ & $\lambda=\frac{\pi}{2}$ & $\lambda=\frac{\pi}{3}$ & $\lambda=\frac{\pi}{4}$ \\
\hline 6 & 0.03512818236443 & 0.06453944018973 & 0.11709717611811 & 0.16640120658498 \\
10 & 0.03477636309547 & 0.06406728710673 & 0.11638357857966 & 0.16544120021330 \\
18 & 0.03464007644564 & 0.06388499113569 & 0.11610862695902 & 0.16507180196427 \\
34 & 0.03459635046007 & 0.06382657412923 & 0.11602059391022 & 0.16495362789363 \\
66 & 0.03458384713708 & 0.06380987621701 & 0.11599543821154 & 0.16491987454694 \\
130 & 0.03458049496149 & 0.06380539993341 & 0.11598869526491 & 0.16491082900319 \\
$\infty$ & 0.03457933093553 & 0.0638038456134 & 0.11598635395420 & 0.16490768852793 \\
\hline
\end{tabular}

Entretanto é interessante ressaltar que o funcional do integrando da expressão (3.115) é diferente daquele apresentado em [3] na solução do modelo a partir da matriz de transferência linha-para-linha. Todavia, quando atribuímos os mesmos valores para os pesos de Boltzmann, as integrais convergem para o mesmo valor numérico, como deveria ser, pois a energia livre no limite termodinâmico deve independer da particular geometria com que definimos o modelo. 


\subsection{4 $\Delta<-1$}

Para atingirmos este limite é conveniente tomarmos os parâmetros $\lambda$ e $\sigma$ e a variável $\sigma^{R}$ como imaginários puros, ou seja,

$$
\lambda \rightarrow-i \lambda, \quad \sigma \rightarrow-i \sigma, \quad \sigma^{R} \rightarrow-i \sigma^{R} .
$$

Fazendo estas substituições em (3.107) a equação integral, no regime em questão, assume a forma

$$
\begin{aligned}
\rho\left(\sigma^{R}\right)= & \frac{1}{2 \pi} \frac{\sinh (\sigma+\lambda)}{\cosh (\sigma+\lambda)-\cos \left(2 \sigma^{R}\right)}+\frac{1}{2 \pi} \frac{\sinh (\lambda-\sigma)}{\cosh ((\lambda-\sigma))-\cos \left(2 \sigma^{R}\right)} \\
& -\frac{1}{\pi} \int_{-Q}^{Q} \frac{\sinh (2 \lambda)}{\cosh (2 \lambda)-\cos \left(2\left(\sigma^{R}-\sigma^{R^{\prime \prime}}\right)\right)} \rho\left(\sigma^{R^{\prime \prime}}\right) d \sigma^{R^{\prime \prime}} .
\end{aligned}
$$

Definindo-se

$$
\bar{\rho}_{m}=\frac{1}{2 \pi} \int_{-\pi}^{\pi} \rho\left(\sigma^{R}\right) e^{i m \sigma^{R}} d \sigma^{R}, \quad \text { e, } 2 \pi \bar{\rho}_{m}=\bar{\varphi}_{m},
$$

a equação (3.118) assume a seguinte forma em termos da variável $m$,

$$
\bar{\varphi}_{m}=\frac{1}{2} e^{-(\sigma+\lambda) \frac{|m|}{2}}+\frac{1}{2} e^{-(\lambda-\sigma) \frac{|m|}{2}}-e^{-\lambda|m|} \bar{\varphi}_{m} .
$$

Isolando-se $\bar{\varphi}_{m}$ nesta última equação, obtemos

$$
\bar{\varphi}_{m}=\frac{\cosh \left(\frac{\sigma m}{2}\right) \operatorname{sech}\left(\frac{\lambda m}{2}\right)}{2} .
$$

-Localização do setor de maior autovalor 
Da última expressão extraímos que $\bar{\varphi}_{0}=\frac{1}{2}$. Além disso sabemos que a transformação de $\varphi\left(\sigma^{R}\right)$ para $\bar{\varphi}_{m}$ é

$$
\bar{\varphi}_{m}=\frac{1}{2 \pi} \int_{-\pi}^{\pi} \varphi\left(\sigma^{R}\right) e^{i m \sigma^{R}} d \sigma^{R}
$$

e que a normalização para $\varphi\left(\sigma^{R}\right)$ é escrita como,

$$
\frac{1}{2 \pi} \int_{-\pi}^{\pi} \varphi\left(\sigma^{R}\right) d \sigma^{R}=\frac{n}{2 N}
$$

A partir de (3.122) e (3.123), vemos que $\bar{\varphi}_{0}=\frac{1}{2}$, e deste modo, o maior autovalor deve se localizar no setor com $n=N$ flechas.

\section{-Energia livre}

A energia livre no limite termodinâmico assume a forma

$$
f_{\infty}=-\frac{k_{B} T}{\pi} \int_{-\pi}^{\pi} \ln \left[\Lambda\left(\sigma^{R}\right)\right] \varphi\left(\sigma^{R}\right) d \sigma^{R},
$$

Se expardirmos $\ln \left[\Lambda\left(\sigma^{R}\right)\right]$ em potências de $e^{i \sigma^{R}}$, isto é,

$$
\ln \left[\Lambda\left(\sigma^{R}\right)\right]=\sum_{m=0}^{\infty} g_{m} e^{i m \sigma^{R}}
$$

a energia livre pode ser escrita como

$$
f_{\infty}=\frac{k_{B} T}{\pi} \sum_{m=0}^{\infty} \int_{-\pi}^{\pi} g_{m} e^{i m \sigma^{R}} \varphi\left(\sigma^{R}\right) d \sigma^{R}=k_{B} T 2 \sum_{m=0}^{\infty} g_{m} \bar{\varphi}_{m}
$$

Fazendo-se explicitamente a expansão de $\ln \left[\Lambda\left(\sigma^{R}\right)\right]$ obtemos finalmente 


$$
\begin{aligned}
& f_{\infty}=-k_{B} T\left[\sigma+\sum_{m=1}^{\infty} \frac{(-1)^{m}}{m} \sinh (2 m \sigma) \operatorname{sech}(m \lambda) e^{-m \lambda}\right], \\
& \lambda>0, \quad-\lambda<\sigma<\lambda .
\end{aligned}
$$

\subsection{Conteúdo operatorial do modelo de 6 vértices a partir da matriz de transferência diagonal-para diagonal}

Os autovalores da matriz de transferência diagonal-para-diagonal estudados nesta dissertação nos permitiram obter os expoentes críticos do modelo de 6 vértices. Estas estimativas são possíveis graças às consequências advindas da invariância conforme dos modelos críticos. Mostraremos que as dimensões anômalas do modelo de seis vértices, calculadas mediante a análise das correções de tamanho finito da matriz de transferência diagonal-para-diagonal, são as mesmas daquelas calculadas usandose a matriz de transferência linha-para-linha. Este fato é esperado pois a teoria de campo associada ao modelo é a mesma independente da matriz transferência usada. Entretanto mostraremos que a velocidade do som, por ser uma quantidade não universal, associada a estas duas matrizes de transferência, assume valores diferentes para o mesmo valor de $\lambda$.

É conveniente redefinirmos os setores da matriz de transferência da seguinte forma

$$
r=N-n, \quad n=0, \ldots, 2 N .
$$

Nesta nova nomenclatura o setor de maior autovalor da matriz de transferência localiza-se em $r=0$. Restrir-nos-emos aos casos em que $r \geq 0$, uma vez que o espectro da matriz de transferência é simétrico pela inversão $r \rightarrow-r$.

Para podermos calcular a velocidade do som $\zeta$ e a dimensão dos operadores $x$ que governam a teoria de campos adjacente ao modelo crítico em estudo, fazemos uso 
das relações advindas da invariância conforme do sistema infinito $(N \rightarrow \infty)$. Conforme mencionamos na introdução desta dissertação (veja relações (1.5) e (1.6)) as quantidades de interesse são obtidas pelas correções devido à finitude da rede, do comportamento assintótico das energias $f_{j, j^{\prime}}^{i}$, do Hamiltonio associado à $T_{D-D}$, isto é, $H=-\ln T_{D-D}$. Denotando por $f_{0}(N, \lambda, \sigma)$ a energia correspondente ao maior autovalor de $T_{D-D}$ (menor autovalor de $H$ ) numa rede de tamanho $N$ e $f_{j, j^{j}}^{i}(N, \lambda, \sigma)$ as energias relacionadas às torres conformes do operador de dimensão $x_{i}$ e spin $s_{i}$, podemos re-escrever as relações (1.5) e (1.6) como

$$
\begin{aligned}
& \frac{f_{0}(N, \lambda, \sigma)}{N}=f_{\infty}(\lambda, \sigma)-\frac{\pi \zeta(\lambda, \sigma) c}{6 N^{2}}+o\left(N^{-2}\right), \\
& f_{j, j^{\prime}}^{i}(N, \lambda, \sigma)=f_{0}(N, \lambda, \sigma)+\frac{2 \pi \zeta(\lambda, \sigma)}{N}\left(x_{i}+j+j^{\prime}\right)+o\left(N^{-1}\right), \\
& f_{j, j^{\prime}}^{i}(N, \lambda, \sigma)=\frac{2 \pi}{N}\left(s_{i}+j-j^{\prime}\right), \quad j, j^{\prime}=0,1,2 \ldots
\end{aligned}
$$

Ao invés de calcularmos a velocidade do som $\zeta$ usando-se os gaps oriundos do primeiro estado excitado de $H$ com momento $\frac{2 \pi}{N}$, como é o procedimento usual [19], usaremos apenas os estados fundamentais dos diversos setores. Para isto, assumiremos como bem estabelecido o valor da anomalia conforme do modelo $c=1$. A velocidade do som $\zeta(N, \lambda, \sigma)$ será obtida pelo limite assintótico $(N \rightarrow \infty)$ da sequência

$$
\zeta(N, \lambda, \sigma)=\frac{6 N}{\pi}\left[f_{\infty}(\lambda, \sigma) N-f_{0}(N, \lambda, \sigma)\right]
$$

Na tabela II mostramos os valores obtidos para $\zeta(N, \lambda, \sigma=0.5)$ para alguns tamanhos de rede. As energias foram obtidas resolvendo-se as equações do ansatz de Bethe. Repare que os resultados calculados são distintos da velocidade do som $\zeta_{X X Z}(\lambda)=\frac{\pi \sin (\lambda)}{\lambda}$ associado com a matriz de transferência linha-para-linha do modelo [19]. Na última linha da tabela II mostramos para efeito de comparação, os valores de $\zeta_{X X Z}(\lambda)$. Tal discordância, atesta a dependência da velocidade do som com as diferentes formas da matriz de transferência 


\section{Tabela II}

\begin{tabular}{|c|c|c|c|c||}
\hline $\mathrm{N}$ & $\lambda=\frac{2 \pi}{3}$ & $\lambda=\frac{\pi}{2}$ & $\lambda=\frac{\pi}{3}$ & $\lambda=\frac{\pi}{4}$ \\
\hline 6 & 0.03773624454706 & 0.05057575758530 & 0.07637450613819 & 0.10268673755431 \\
10 & 0.03763037006426 & 0.05031361904847 & 0.07586431519232 & 0.10189322630966 \\
18 & 0.03758898261725 & 0.05021239627931 & 0.07566185294259 & 0.10155247844489 \\
34 & 0.03757566152908 & 0.05017995741567 & 0.075594884866292 & 0.10142481079232 \\
66 & 0.03757184873142 & 0.05017068491387 & 0.07557509071692 & 0.10137972294200 \\
130 & 0.03757082640365 & 0.05016819918004 & 0.07556960190088 & 0.10136393437831 \\
$\zeta_{X X Z}(\lambda)$ & 1.299038106 & 2.0 & 2.598076211 & 2.8284427125 \\
\hline
\end{tabular}

\subsubsection{Velocidade do som em $\Delta=0$}

Para calcular a velocidade do som de forma analítica neste regime, partimos do conhe- cimento das soluções das equações de Bethe, correspondente aos estados de maior autovalor real com momentos nulo, e $P=\frac{2 \pi}{N}$. Tomando o logarítmo destes autovalores e expandindo o resultado para grandes valores de $N$ obtemos o seguinte resultado

$$
f_{P=\frac{2 \pi}{N}}-f_{0} \approx \frac{\pi \tan (\sigma)}{N} .
$$

Como a relação de dispersão energia-momento nos diz que

$$
f_{P}-f_{0} \approx \frac{P \zeta}{N},
$$

podemos concluir da última equação que

$$
\zeta\left(\lambda=\frac{\pi}{2}, \sigma\right)=\frac{\tan (\sigma)}{2} .
$$


Mostramos na tabela III o resultado do cálculo numérico da velocidade do som em $\lambda=\frac{\pi}{2}$, para diferentes valores de $\sigma$, numa rede de $N=130$ sítios, em comparação com a expressão acima obtida $N \rightarrow \infty$.

\section{Tabela III}

\begin{tabular}{|c|c|c|}
\hline$\sigma$ & $\zeta_{\text {Teórico }}$ & $\zeta_{\text {Medido }}$ \\
\hline 0.1 & 0.05016733604273 & 0.05016819918004 \\
0.2 & 0.10135501775434 & 0.10135681533005 \\
0.3 & 0.15466812480481 & 0.15467101176225 \\
0.4 & 0.21139660936908 & 0.21140085411090 \\
0.5 & 0.27315124492190 & 0.27315728665622 \\
\hline
\end{tabular}

\subsubsection{Estados "excitados"}

Assim como na análise da matriz de transferência linha-para-linha, esperamos que parte das dimensões $x_{i}$ associados aos operadores $O_{i}$ varie continuamente com $\lambda$. Na literatura [19] tais dimensões são parametrizados em termos de pares de inteiros $(r, s)$, ou seja,

$$
\begin{aligned}
& x_{r, s}=r^{2} x_{p}+\frac{s^{2}}{4 x_{p}}, \quad \text { com } \\
& x_{p}=\frac{\pi-\lambda}{2 \pi}, \quad r, s=0,1, \ldots ;(r, s) \neq(0,0) .
\end{aligned}
$$

Além dos operadores acima mencionados o modelo exibe também operadores com dimensões independentes de $\lambda$, que correspondem a inteiros,

- Maior autovalor em um setor com $r$ flechas: Vamos calcular a dimensão $x_{r, 0}$ associada ao operador $O_{r, 0}$. Para isto usaremos a segunda das equações (3.129) para formar a sequência. 


$$
\Gamma_{N}(\lambda, \sigma, r)=\frac{N}{2 \pi}\left[f_{0,0}^{r}(N, \lambda, \sigma)-f_{0}(N, \lambda, \sigma)\right]
$$

que deve, no limite $N \rightarrow \infty$, convergir para $\zeta(\lambda, \sigma) x_{r, 0}$. Em (3.135) $f_{0,0}^{r}$ está associado ao maior autovalor da matriz de transferência no setor com $r$ flechas. Como não sabemos a forma analítica de $\zeta(\lambda, \sigma)$ para $\Delta \neq 0$, vamos aproximar $\zeta(\lambda, \sigma)$ por $\zeta_{N=130}(\lambda, \sigma)$ para que possamos obter uma boa estimativa da dimensão $x_{r, 0}$. Na tabela IV listamos $\frac{\Gamma(\lambda, \sigma, 2)}{\zeta_{N=130}(\lambda, \sigma)}$, para diversos valores de $\lambda$ e $N=$ tomando o parâmetro $\sigma=0.1$. Vemos que o comportamento assintótico dos dados desta última tabela indicam que $\frac{x_{r, 0}}{4}=x_{p}$, sendo $x_{p}$ dado pela expressão (3.134). Para efeito de comparação exibimos na última linha da tabela IV os valores esperados de $x_{p}$ fornecidos por (3.135).

Expomos na figura $3.13 \mathrm{em}$ anexo os quocientes $\frac{\Gamma(\lambda, \sigma, r)}{\zeta_{N=130}(\lambda, \sigma)}$ para $r=1,2,3$ e 4 . Para simples visualização mostramos tais dados como função de $x_{p}$, para a cadeia de $N=66$ sítios. Os resultados da figura, assim como diversos outros resultados numéricos que não apresentaremos por brevidade, indicam que

$$
x_{r, 0}=r^{2} x_{p},
$$

em concordância com (3.134).

\section{Tabela IV}




\begin{tabular}{|c|c|c|c|c|}
\hline $\mathrm{N}$ & $\lambda=\frac{2 \pi}{3}$ & $\lambda=\frac{\pi}{2}$ & $\lambda=\frac{\pi}{3}$ & $\lambda=\frac{\pi}{4}$ \\
\hline 6 & 0.16676687232160 & 0.24712196038398 & 0.32156619179235 & 0.35288775675184 \\
10 & 0.16669813477183 & 0.24895867880621 & 0.32848712046665 & 0.36424301190758 \\
18 & 0.16667118822612 & 0.24967533316320 & 0.33163455071116 & 0.37031655619353 \\
34 & 0.16666377767609 & 0.24990588088981 & 0.33279409867774 & 0.37307026127719 \\
66 & 0.16666111002548 & 0.24997186077500 & 0.33308163423995 & 0.37421361133470 \\
130 & 0.16666077565395 & 0.24998955435956 & 0.33328029440123 & 0.37467033465614 \\
$x_{p}$ & $0.1666 .$. & 0.25 & $0.3333 .$. & 0.375 \\
\hline
\end{tabular}

- $O$ operador marginal

Conforme já comentado, além dos operadores $O_{r, s}$ cujas dimensões $x_{r, s}$ variam continuamente, existem também na teoria de campos que governam as flutuações do modelo, operadores cujas dimensões são independentes de $\lambda$. O mais importante deles é o operador marginal [24], com dimensão $x_{m}=2$, que deve governar a variação dos expoentes críticos ao longo da linha crítica $-1<\Delta<1$. O nível mais baixo da torre conforme correspondente a este operador $\left(j=j^{\prime}=0\right)$ em (3.129) corresponde ao estado que chamamos na seção (3.4.1.1) por "estado marginal" e cuja distribuição de raízes foi apresentada. Este estado é obtido no setor com $n=N$ flechas $(r=0)$. Na tabela $\mathrm{V}$ mostramos as estimativas para $x_{m}$ obtidas para alguns valores de $N$ e para dois valores ${ }^{7}$ de $\lambda$. Verificamos nesta tabela claramente o comportamento assintótico $x_{m}=2$.

\section{Tabela V}

\footnotetext{
${ }^{7}$ Infelizmente, devido à instabilidade numérica, não conseguimos calcular a solução das equações do ansatz de Bethe correspondente ao estado marginal para $\lambda<\frac{\pi}{3}$ ou $\lambda>\frac{\pi}{2}$.
} 


\begin{tabular}{|c|c|c|}
\hline $\mathrm{N}$ & $\lambda=\frac{\pi}{2}$ & $\lambda=\frac{2 \pi}{5}$ \\
\hline 10 & 1.99166943044940 & 1.97200601639985 \\
18 & 1.99740266530500 & 1.99112675568633 \\
34 & 1.99924704711735 & 1.99745305651820 \\
66 & 1.99977488619794 & 1.99929004361748 \\
130 & 1.99991643487650 & 1.99978661156063 \\
\hline
\end{tabular}




\section{Capítulo 4}

\section{Modelos de 10 vértices exatamente integrável na rede diagonal}

\subsection{Descrição do modelo}

No capítulo anterior introduzimos e resolvemos exatamente uma família de modelos de vértices interagentes que incluem o conhecido modelo de 6 vértices [2],[3] como caso particular $\left(s_{1}=s_{2}=\ldots=s_{n}=1\right)$. Neste capítulo consideraremos generalizações do modelo de 6 vértices em que o número de flechas em uma dada linha definindo um vértice pode ser maior que a unidade. A generalização mais simples, e que trataremos, são os modelos de 10 vértices. Em tais modelos permitimos duplas ocupações de flechas para as linhas verticais e apenas simples ocupações de flechas para as linhas horizontais que definem os vértices. Assim como no modelo de 6 vértices, os vértices devem satisfazer à regra do gelo, isto é, o número de flechas entrando e saindo em um dado vértices deve ser igual. O número de configurações de vértices satisfazendo tal regra é 10, e na figura 4.1 mostramos as possíveis configurações com os respectivos valores das fugacidades. Sem perda de generalidade, assumiremos $a_{0}=1$. Repare que o caso particular $a_{2}=a_{3}=b_{3}=c_{3}=0$ recai no tradicional modelo de 6 vértices, já discutido anteriormente. 
O nosso objetivo neste capítulo é determinar as possíveis relações entre as fugacidades exibidas na figura 4.1 que tornam o modelo exatamente integrável. Tal tarefa, de forma análoga ao que fizemos no capítulo 3, será executada utilizando-se a matriz de transferência diagonal-para-diagonal associada à uma rede $N \times M$, com condições de contorno especiais. ${ }^{1}$
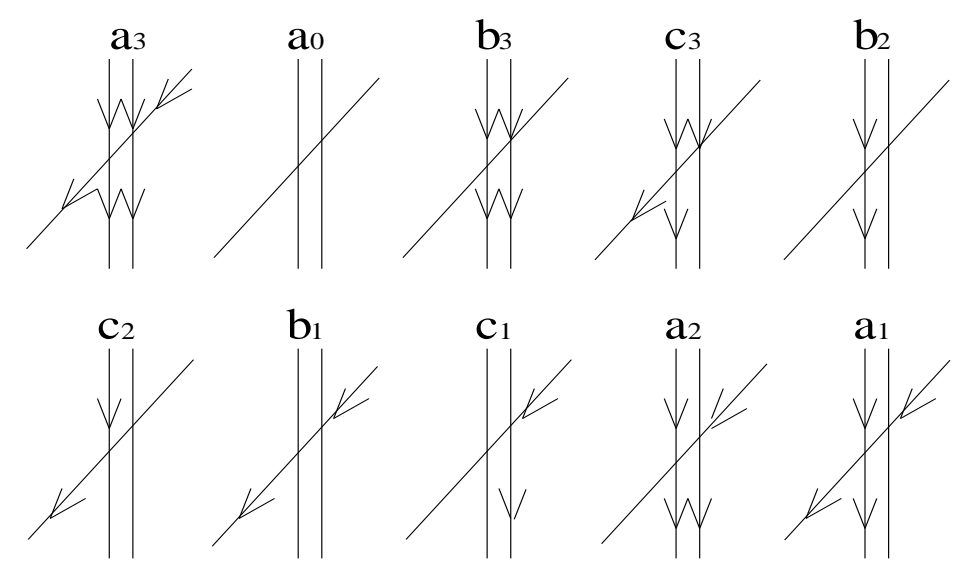

Figura 4.1: Vértices do modelo de 10 vértices.

\subsection{Diagonalização da matriz de transferência diagonal- para-diagonal associada ao modelo de 10 vértices}

Analogamente ao que fizemos no modelo de 5 vértices interagentes iremos considerar nosso modelo na rede quadrada deformada introduzida no capítulo 2. A matriz de transferência diagonal-para-diagonal é então subdividida em blocos diagonais disjuntos indexados pelo número $n$ de flechas numa dada linha da rede deformada. A equação de autovalor no setor de $n$ flechas é dada por

$$
\Lambda_{n}\left|\Psi_{n}>=T_{D-D}\right| \Psi_{n}>
$$

\footnotetext{
${ }^{1}$ Veja o capítulo 2 para a introdução da rede quadrada deformada e da matriz de transferência diagonalpara-diagonal.
} 
sendo $\Lambda_{n}$ e $\mid \Psi_{n}>$ os autovalores e autovetores, respectivamente. Os autovetores $\mid \Psi_{n}>$ possuem a forma geral

$$
\left|\Psi_{n}\right\rangle=\sum_{\substack{1 \leq x_{1} \leq x_{2} \leq \cdots \leq x_{n} \leq N \\ \phi_{\alpha_{1}, \alpha_{2}, \ldots, \alpha_{n}}\left(x_{1}, x_{2}, \ldots, \alpha_{n}, \ldots, x_{n}\right) \mid x_{1}, \alpha_{1} ; x_{2}, \alpha_{2} ; \ldots ; x_{n}, \alpha_{n}>.}}^{2 *} .
$$

onde $\phi_{\alpha_{1}, \alpha_{2}, \ldots, \alpha_{n}}\left(x_{1}, x_{2}, \ldots, x_{n}\right)$ são as amplitudes correspondentes às configurações em que flechas dos tipos $\left(\alpha_{1}, \alpha_{2}, \ldots, \alpha_{n}\right)$ estejam localizadas em $\left(x_{1}, x_{2}, \ldots, x_{n}\right)$. O símbolo * em (4.2) indica que as configurações devem satisfazer à regra de não haver mais que uma (duas) flechas do tipo 2 (tipo 1) associada à mesma coordenada de posição $x$.

Analogamente ao que fizemos no capítulo 3 tentaremos obter as soluções dos modelos utilizando, ao invés do ansatz de Bethe tradicional, o ansatz do produto matricial. A formulação deste ansatz segue de uma simples generalização daquele que formulamos para o caso do modelo de 5 vértices interagentes (veja capítulo 3). Associamos à amplitude $\phi_{\alpha_{1}, \alpha_{2}, \ldots, \alpha_{n}}\left(x_{1}, x_{2}, \ldots, x_{n}\right)$ o traço de um produto de matrizes. $\mathrm{Na}$ composição de tal produto associamos distintas matrizes, com álgebra a ser determinada, aos sítios da rede, de acordo com a ocupação dos mesmos. Na figura 4.2 mostramos as possíveis ocupações do sítios, bem como a matriz associada para a formulação de nosso ansatz do produto matricial. As amplitudes associadas à configuração em que não haja dupla ocupação de flechas do tipo 1, são dadas de forma idêntica à associação do modelo de 5 vértices interagente, isto é:

$$
\begin{aligned}
& \phi_{\alpha_{1}, \alpha_{2}, \ldots, \alpha_{n}}\left(x_{1}, x_{2}, \ldots, x_{n}\right)= \\
& \operatorname{Tr}\left[E^{x_{1}-1} A^{\left(\alpha_{1}\right)} E^{x_{2}-x_{1}-1} A^{\left(\alpha_{2}\right)} \cdots E^{x_{n}-x_{n-1}} A^{\left(\alpha_{n}\right)} E^{N-x_{n}} \Omega_{P}\right],
\end{aligned}
$$

ao passo que se tivermos uma ocupação dupla, isto é, $x_{i+1}=x_{i}$ de flechas do tipo 1 
$\left(\alpha_{i+1}=\alpha_{i}=1\right)$ teremos a associação

$$
\begin{aligned}
& \phi_{\alpha_{1}, \ldots, \alpha_{i}, \alpha_{i+1}, \ldots, \alpha_{n}}\left(x_{1}, \ldots, x_{i}, x_{i+1}, \ldots, x_{n}\right)= \\
& \operatorname{Tr}\left[E^{x_{1}-1} A^{\left(\alpha_{1}\right)} \cdots E^{x_{i}-x_{i-1}-1} A^{(3)} \cdots E^{x_{i+2}-x_{i}-1} A^{\left(\alpha_{i+2}\right)} \cdots E^{x_{n}-x_{n-1}} A^{\left(\alpha_{n}\right)} E^{N-x_{n}} \Omega_{P}\right] .
\end{aligned}
$$

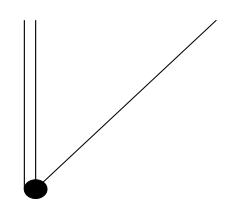

$\mathrm{E}$

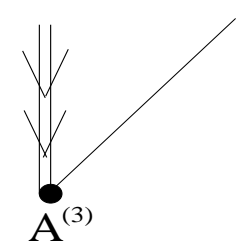

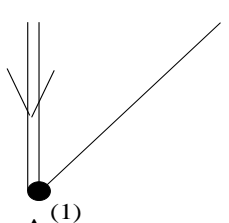

$\mathrm{A}^{(1)}$

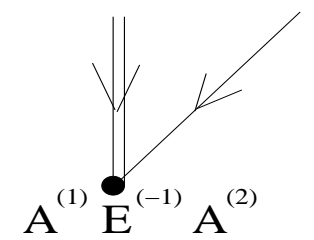

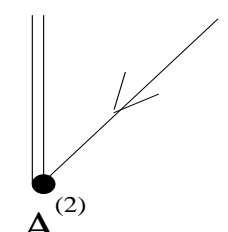

$\mathrm{A}^{(2)}$

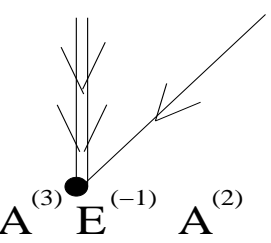

Figura 4.2: Vértices do modelo de 10 vértices.

O produto de matrizes associado às configurações gerais segue de (4.3) e (4.4). Em (4.3) e (4.4) a matriz $\Omega_{P}$ foi introduzida pelo fato de querermos produzir autofunções de $T_{D-D}$ que também o sejam do operador de translação (momento) ao longo da diagonal da rede (comprimento $N$ ). Tal operador comuta com $T_{D-D}$, devido às condições periódicas de contorno ao longo da diagonal e seus autovalores são dados por $P=\frac{2 \pi}{N} j(j=0,1, \ldots, N-1)$. A propriedade cíclica do traço nos garante que (4.3) e (4.4) serão também autofunções do operador momento, caso $\Omega_{P}$ satisfaça às relações algébricas

$$
E \Omega_{P}=e^{-i P} \Omega_{P} E, \quad A^{(j)} \Omega_{P}=e^{-i P} \Omega_{P} A^{(j)}, \quad j=1,2,3 .
$$

Ao considerarmos o nosso ansatz (4.3) e (4.4) em setores com poucas flechas, ve- 
rificamos que analogamente ao ocorrido na solução dos modelos de 5 vértices interagentes, as matrizes $A^{(\alpha)}(\alpha=1,2,3)$ em setores $\operatorname{com} n$ flechas são formadas por combinações de matrizes espectrais, isto é:

$$
A^{(j)}=\sum_{i=1}^{n} \phi_{j}^{i} A_{k_{j}} E(j=1,2) ; \quad A^{(3)}=\sum_{i, j=1, i \neq j}^{n} \phi_{1}^{i} \phi_{1}^{j} B_{k_{i}, k_{j}} E
$$

sendo que as matrizes espectrais $A_{k_{j}}$ e $B_{k_{i}, k_{j}}$ dependem dos mesmos parâmetros espectrais $\left(k_{1}, \ldots, k_{n}\right)$ e satisfazem à seguinte relação de comutação com o operador $E$.

$$
\begin{aligned}
& E A_{k_{l}}=e^{i k_{l}} A_{k_{l}} E, \quad(l=1,2, \ldots, n), \\
& E B_{k_{j}, k_{l}}=e^{i\left(k_{j}+k_{l}\right)} B_{k_{j}, k_{l}} E, \quad(j \neq l=1,2, \ldots, n) .
\end{aligned}
$$

Repare que (4.5), (4.6) e (4.7) implicam em

$$
\left[A_{k_{l}}, \Omega_{P}\right]=\left[B_{k_{j}, k_{l}}, \Omega_{P}\right]=0, \quad j, l=1,2, \ldots, n
$$

Passaremos, a seguir, à diagonalização do modelo iniciando pelos setores com poucas flechas, para posteriormente apresentarmos o resultado para $n$ geral.

- Setor $\operatorname{com} n=0$ ou $n=1$

Nos casos em que $n=0$ ou $n=1$ nosso modelo é idêntico ao caso particular do modelo interagente considerado no capítulo anterior, em que todas as flechas possuem tamanho $s=1$. Neste caso as equações (3.10)-(3.22) seriam as mesmas para o presente modelo.

- setor com n=2 flechas

As equações provenientes das configurações em que as duas flechas não estejam em posição de colisão são as mesmas equações (veja (3.23)-(3.26)) obtidas no capítulo 
anterior para o caso em que $s_{1}=s_{2}=1$. Desta forma, a estrutura do autovalor neste setor é a mesma do modelo de 5 vértices interagentes, isto é,

$$
\Lambda_{2}^{l_{1}, l_{2}}=\Lambda_{1}^{l_{1}}\left(k_{1}\right) \Lambda_{1}^{l_{2}}\left(k_{2}\right), \quad l_{1}, l_{2}=+,-,
$$

sendo que $\Lambda_{1}^{l}(k)$ vem do problema de uma flecha, apresentado no problema de 5 vértices interagentes.

A novidade no processo de diagonalização surge quando estudamos as equações provenientes das configurações onde haja colisões entre as duas flechas. Para vermos isto, consideremos as configurações de flechas apresentadas nas figuras 4.3a e 4.3b.

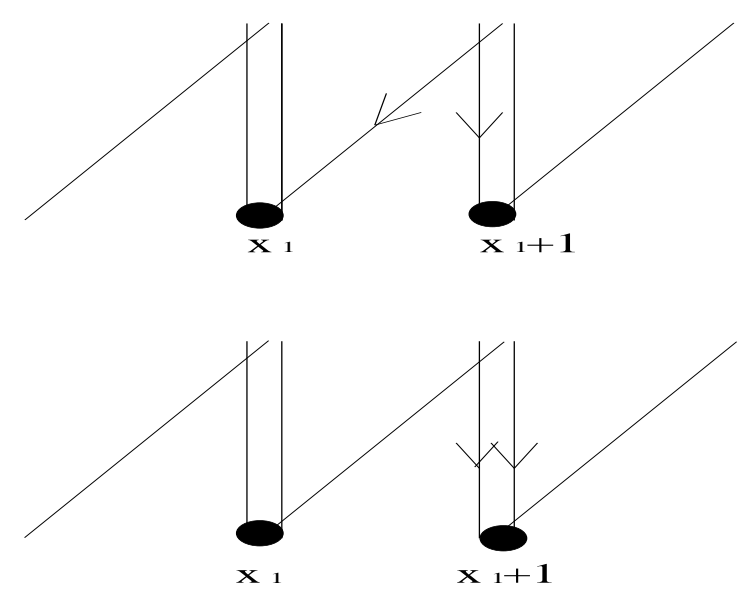

(a)

(b)

Figura 4.3: Situações de colisão.

As relações advindas das configurações mostradas nas figuras 4.3a e 4.3b são escritas, respectivamente como

$$
\begin{aligned}
& \Lambda_{2} \phi_{21}\left(x_{1}, x_{1}+1\right)=a_{1} \phi_{12}\left(x_{1}+1, x_{1}+1\right)+c_{3} \phi_{11}^{1 * 2}\left(x_{1}+1, x_{1}+1\right), \\
& \Lambda_{2} \phi_{11}^{1 * 2}\left(x_{1}, x_{1}\right)=b_{3} \phi_{11}^{1 * 2}\left(x_{1}, x_{1}\right)+a_{2} \phi_{12}\left(x_{1}, x_{1}\right)
\end{aligned}
$$

onde denotamos por $\phi_{11}^{i * i+1}$ a amplitude correspondente à configuração em que duas flechas do tipo 1 estão localizadas em um mesmo sítio. O ansatz matricial (4.3) e (4.4) utilizado na primeira equação do conjunto (4.10) nos dá 


$$
\begin{aligned}
& \Lambda_{2} \operatorname{tr}\left[E^{x_{1}-1} A^{(2)} A^{(1)} E^{N-x_{1}-1} \Omega_{P}\right]=a_{1} \operatorname{tr}\left[E^{x_{1}} A^{(1)} E^{-1} A^{(2)} E^{N-x_{1}-1} \Omega_{P}\right] \\
& +c_{3} \operatorname{tr}\left[E^{x_{1}-1} A^{(3)} E^{N-x_{1}} \Omega_{P}\right] .
\end{aligned}
$$

Substituindo $A^{(\alpha)}(\alpha=1,2,3)$ pelas matrizes espectrais dadas em (4.6) obtemos

$$
\begin{aligned}
& \sum_{i, j=1}^{2} e^{i k_{j}}\left(\Lambda_{2} \phi_{2}^{i} \phi_{1}^{j}-a_{1} \phi_{1}^{i} \phi_{2}^{j} e^{i k_{i}}\right) \operatorname{tr}\left[E^{x_{1}-1} A_{k_{i}} A_{k_{j}} E^{N-x_{1}+1} \Omega_{P}\right]= \\
& c_{3} \sum_{i, j=1}^{2} \phi_{1}^{i} \phi_{1}^{j} e^{i\left(k_{i}+k_{j}\right)} \operatorname{tr}\left[E^{x_{1}-1} B_{k_{i}, k_{j}} E E^{N-x_{1}+1} \Omega_{P}\right], \quad x_{2}=x_{1}+1,
\end{aligned}
$$

onde as relações de comutação (4.7) foram usadas. Da mesma forma, a segunda equação do conjunto (4.10) torna-se

$$
\begin{aligned}
& \sum_{i, j=1}^{2} \operatorname{tr}\left[E^{x_{1}-1} \phi_{1}^{i} \phi_{1}^{j} B_{k_{i}, k_{j}} E^{N-x_{1}+1} \Omega_{P}\right]\left(\Lambda_{2}-b_{3}\right)= \\
& a_{2} \sum_{i, j=1}^{2} \operatorname{tr}\left[E^{x_{1}-1} \phi_{1}^{i} \phi_{2}^{j} A_{k_{i}} A_{k_{j}} E^{N-x_{1}+1} \Omega_{P}\right] .
\end{aligned}
$$

Multiplicando-se (4.12) por $\left(\Lambda_{2}-b_{3}\right)$ e (4.13) por $c_{3}$ podemos eliminar os traços envolvendo a matriz $B_{k_{i}, k_{j}}$ e obtemos

$$
\begin{aligned}
& \left(\Lambda_{2}-b_{3}\right) \sum_{i, j=1}^{2} e^{i\left(k_{i}+k_{j}\right)}\left(\Lambda_{2} \phi_{2}^{i} \phi_{1}^{j}-a_{1} \phi_{1}^{i} \phi_{2}^{j} e^{i k_{i}}\right) \operatorname{tr}\left[E^{x_{1}-1} A_{k_{i}} A_{k_{j}} E^{2} E^{N-x_{1}-1} \Omega_{P}\right]= \\
& c_{3} a_{2} \sum_{i, j=1}^{2} \operatorname{tr}\left[E^{x_{1}-1} \phi_{1}^{i} \phi_{2}^{j} A_{k_{i}} A_{k_{j}} E^{2} E^{N-x_{1}-1} \Omega_{P}\right] .
\end{aligned}
$$

Das relações obtidas na situação de duas flechas não interagentes, totalmente análogas às obtidas para o modelo considerado no capítulo anterior, extraímos que os escalares $\phi_{\alpha}^{i}$ e as funções $\Lambda\left(k_{i}\right)$ satisfazem 


$$
\begin{gathered}
\phi_{2}^{i} \phi_{1}^{j}=\frac{\Lambda\left(k_{i}\right)-b_{2}}{\Lambda\left(k_{j}\right)-b_{2}} \phi_{2}^{j} \phi_{1}^{i}, \quad \phi_{2}^{j}=\frac{\left(\Lambda\left(k_{j}\right)-b_{2}\right) \phi_{1}^{j}}{c_{1}}, \\
e^{-i k_{i}} \Lambda k_{i}-e^{-i k_{i}} \Lambda\left(k_{i}\right) b_{2}=\Lambda\left(k_{i}\right) b_{1}-\left(b_{2} b_{1}-c_{2} c_{1}\right) .
\end{gathered}
$$

Se substituirmos estas relações na equação (4.14) obtemos

$$
\begin{aligned}
& \sum_{i, j=1}^{2} \frac{\phi_{1}^{j} \phi_{1}^{i}}{c_{1}}\left\{\left[\left(\Lambda\left(k_{i}\right) b_{1}+q_{1}\right) \Lambda\left(k_{j}\right)-a_{1}\left(\Lambda\left(k_{j}\right)-b_{2}\right)\right] \cdot\left(\Lambda\left(k_{i}\right) \Lambda\left(k_{j}\right)-b_{3}\right)\right. \\
& \left.-q_{2}\left(\Lambda\left(k_{i}\right)-b_{2}\right)\right\} \operatorname{tr}\left[E^{x_{1}-1} A_{k_{i}} A_{k_{j}} E^{N-x_{1}+1} \Omega_{P}\right]=0,
\end{aligned}
$$

onde, $q_{1}=c_{1} c_{2}-b_{2} b_{1}, q_{2}=a_{2} c_{3}$. Para que a equação (4.16) seja verdadeira para todo $x_{1}$, devemos impor que as matrizes $A_{k_{i}}, A_{k_{j}},(i \neq j)$ satisfaçam à seguinte relação de comutação

$$
\begin{aligned}
& A_{k_{i}} A_{k_{j}}=\frac{\alpha\left(k_{j}, k_{i}\right)}{\alpha\left(k_{i}, k_{j}\right)} A_{k_{j}} A_{k_{i}}, \quad A_{k_{i}}^{2}=0 \\
& \alpha\left(k_{j}, k_{i}\right)= \\
& {\left[\left(\Lambda\left(k_{j}\right) b_{1}+q_{1}\right) \Lambda\left(k_{j}\right)-a_{1}\left(\Lambda\left(k_{j}\right)-b_{2}\right)\right]\left[\left(\Lambda\left(k_{i}\right) \Lambda\left(k_{j}\right)-b_{3}\right)-a_{2} c_{3}\left(\Lambda\left(k_{j}\right)-b_{2}\right)\right] .}
\end{aligned}
$$

Os parâmetros $\left(k_{1}, k_{2}\right)$ são livres até este momento. A propriedade cíclica do traço junto com as relações de comutação entre as matrizes $A_{k_{1}}, A_{k_{2}}, \Omega_{P}$ e $E$ dadas em (4.5) e (4.7), servirão para extrair as equações que fixam os valores de $\left(k_{1}, k_{2}\right)$. Para obter estas equações observamos que

$$
\begin{aligned}
& \operatorname{Tr}\left[A_{k_{i}} A_{k_{j}} E^{N} \Omega_{P}\right]=e^{-i k_{j} N} \operatorname{Tr}\left[A_{k_{i}} E^{N} A_{k_{j}} \Omega_{P}\right]= \\
& e^{-i k_{j} N} \operatorname{Tr}\left[E^{N} A_{k_{j}} \Omega_{P} A_{k_{i}}\right]=e^{-i k_{j} N} s\left(k_{j}, k_{i}\right) \operatorname{Tr}\left[A_{k_{i}} A_{k_{j}} E^{N} \Omega_{P}\right] .
\end{aligned}
$$

Isto nos assegura que os parâmetros espectrais satisfazem 


$$
e^{i k_{j} N}=\frac{\alpha\left(k_{j}, k_{i}\right)}{\alpha\left(k_{i}, k_{j}\right)}, \quad j=1,2, \quad i \neq j .
$$

Os autovalores de $T_{D-D}$ sendo então obtidos pelas dados substituindo-se as raízes de (4.19) em (4.9).

\section{- Setor com $n=3$ flechas}

A análise das configurações em que as três flechas estão separadas, nos fornecem equações similares àquelas obtidas no setor $\operatorname{com} n=2$ flechas. Tais equações podem ser representadas na seguinte forma tensorial

$$
\begin{aligned}
& \Lambda_{3}\left(\begin{array}{l}
\phi_{1}^{1} \\
\phi_{2}^{1}
\end{array}\right) \otimes\left(\begin{array}{l}
\phi_{1}^{2} \\
\phi_{2}^{2}
\end{array}\right) \otimes\left(\begin{array}{c}
\phi_{1}^{3} \\
\phi_{2}^{3}
\end{array}\right)= \\
& \left(\begin{array}{cc}
b_{2} & c_{1} \\
c_{2} e^{i k_{3}} & b_{1} e^{i k_{3}}
\end{array}\right) \otimes\left(\begin{array}{cc}
b_{2} & c_{1} \\
c_{2} e^{i k_{2}} & b_{1} e^{i k_{2}}
\end{array}\right) \otimes\left(\begin{array}{cc}
b_{2} & c_{1} \\
c_{2} e^{i k_{1}} & b_{1} e^{i k_{1}}
\end{array}\right)\left(\begin{array}{l}
\phi_{1}^{1} \\
\phi_{2}^{1}
\end{array}\right) \otimes\left(\begin{array}{l}
\phi_{1}^{2} \\
\phi_{2}^{2}
\end{array}\right) \otimes\left(\begin{array}{l}
\phi_{1}^{3} \\
\phi_{2}^{3}
\end{array}\right) .
\end{aligned}
$$

Se impormos que $\Lambda_{3}=\Lambda\left(k_{1}\right) \Lambda\left(k_{2}\right) \Lambda\left(k_{3}\right)$, as equações acima são separadas em três relações análogas às de uma flecha.

Consideraremos agora as equações provenientes das configurações em que duas flechas estão em posição de "colisão", enquanto que a terceira está separada destas duas. As configurações onde tais colisões aparacem são representadas nas figuras 4.4 - 4.11. A primeira configuração (figura 4.4) produz as seguintes relações entre as amplitudes

$$
\begin{aligned}
& \Lambda_{3} \phi_{121}\left(x_{1}, x_{2}, x_{3}\right)=b_{2} a_{1} \phi_{112}\left(x_{1}, x_{3}, x_{3}\right)+c_{1} a_{1} \phi_{212}\left(x_{1}, x_{3}, x_{3}\right)+ \\
& b_{2} c_{3} \phi_{111}^{2 * 3}\left(x_{1}, x_{3}, x_{3}\right)+c_{1} c_{3} \phi_{211}^{2 * 3}\left(x_{1}, x_{3}, x_{3}\right) .
\end{aligned}
$$

Substituindo o nosso ansatz matricial (4.3) e (4.4) nesta última equação obtemos 


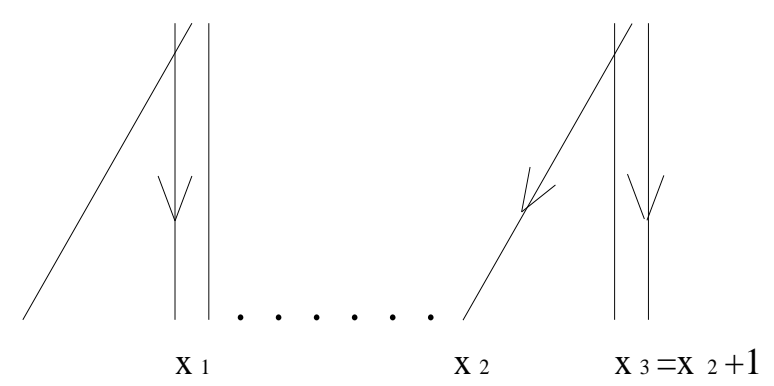

Figura 4.4: Configuração de 2 flechas juntas e uma separada (situação 1).

$$
\begin{aligned}
& \Lambda_{3} \operatorname{tr}\left[E^{x_{1}-1} A^{(1)} E^{x_{2}-x_{1}-1} A^{(2)} E^{x_{3}-x_{2}-1} A^{(1)} E^{N-x_{3}} \Omega_{P}\right]= \\
& b_{2} a_{1} \operatorname{tr}\left[E^{x_{1}-1} A^{(1)} E^{x_{3}-x_{1}-1} A^{(1)} E^{x_{3}-x_{3}-1} A^{(2)} E^{N-x_{3}} \Omega_{P}\right]+ \\
& c_{1} a_{1} \operatorname{tr}\left[E^{x_{1}-1} A^{(2)} E^{x_{3}-x_{1}-1} A^{(1)} E^{x_{3}-x_{3}-1} A^{(2)} E^{N-x_{3}} \Omega_{P}\right]+ \\
& b_{2} c_{3} \operatorname{tr}\left[E^{x_{1}-1} A^{(1)} E^{x_{3}-x_{1}-1} A^{(3)} E^{N-x_{3}} \Omega_{P}\right]+ \\
& c_{1} c_{3} \operatorname{tr}\left[E^{x_{1}-1} A^{(2)} E^{x_{3}-x_{1}-1} A^{(3)} E^{N-x_{3}} \Omega_{P}\right] .
\end{aligned}
$$

Escrevendo as matrizes $A^{(\alpha)}(\alpha=1,2,3)$ em termos das matrizes espectrais $A_{k_{i}}$ e $B_{k_{i}, k_{j}}$ como em (4.6) e utilizando as relações de comutação (4.7) obtemos

$$
\begin{aligned}
& \sum_{i, j, l=1}^{3} \operatorname{tr}\left[E^{x_{1}-1} A_{k_{i}} E^{x_{2}-x_{1}} A_{k_{j}} E A_{k_{l}} E^{N-x_{3}+1} \Omega_{P}\right] \\
& \times\left(\Lambda_{3} \phi_{1}^{i} \phi_{2}^{j} \phi_{1}^{l}-b_{2} a_{1} \phi_{1}^{i} \phi_{1}^{j} \phi_{2}^{l} e^{i k_{j}}-c_{1} a_{1} \phi_{2}^{i} \phi_{1}^{j} \phi_{2}^{l} e^{i k_{j}}\right)= \\
& \sum_{i, j, l=1}^{3} \operatorname{tr}\left[E^{x_{1}-1} A_{k_{i}} E^{x_{2}-x_{1}+1} B_{k_{j}, k_{l}} E^{N-x_{3}+1} \Omega_{P}\right]\left(b_{2} c_{3} \phi_{1}^{i} \phi_{1}^{j} \phi_{1}^{l}+c_{1} c_{3} \phi_{2}^{i} \phi_{1}^{j} \phi_{1}^{l}\right) .
\end{aligned}
$$

A segunda relação advinda da configuração apresentada na figura 4.5 é análoga à situação que analisamos anteriormente, não oferecendo nenhuma relação adicional. Uma nova relação é obtida das configurações exibidas na figura 4.6. As equações para as amplitudes correspondentes relacionadas com esta configuração são 


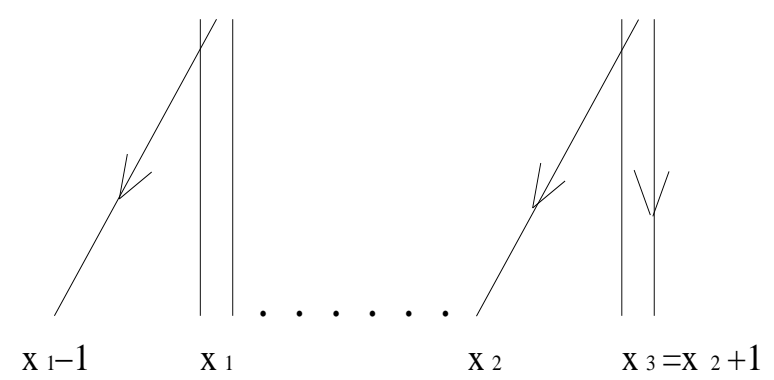

Figura 4.5: Configuração de 2 flechas juntas e uma separada (situação 2).

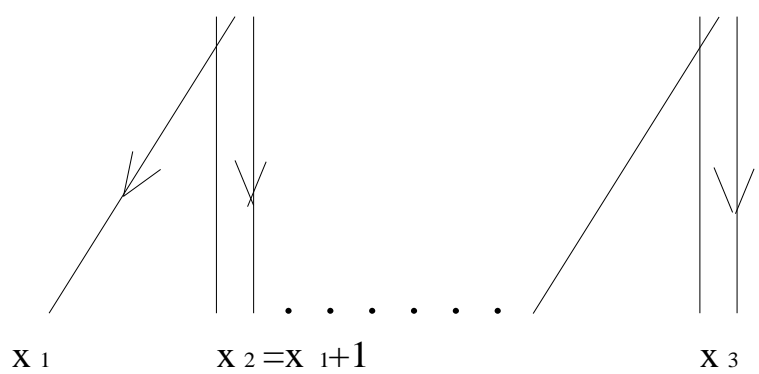

Figura 4.6: Configuração de 2 flechas juntas e uma separada (situação 3).

$$
\begin{aligned}
& \Lambda_{3} \phi_{211}\left(x_{1}, x_{2}, x_{3}\right)=a_{1} b_{2} \phi_{121}\left(x_{2}, x_{2}, x_{3}\right)+a_{1} c_{1} \phi_{122}\left(x_{2}, x_{2}, x_{3}\right)+ \\
& c_{3} b_{2} \phi_{111}^{1 * 2}\left(x_{2}, x_{2}, x_{3}\right)+c_{3} c_{1} \phi_{112}^{1 * 2}\left(x_{2}, x_{2}, x_{3}\right) .
\end{aligned}
$$

Substituindo-se o ansatz matricial (4.3) e (4.4) juntamente com (4.6) nesta última equação e utilizando as relações de comutação (4.7) obtemos depois de uma simplificação

$$
\begin{aligned}
& \sum_{i, j, l=1}^{3}\left(\Lambda_{3} \phi_{1}^{i} \phi_{2}^{j} \phi_{1}^{l}-a_{1} b_{2} \phi_{1}^{i} \phi_{2}^{j} \phi_{1}^{l} e^{i k_{i}}-a_{1} c_{1} \phi_{1}^{i} \phi_{2}^{j} \phi_{2}^{l} e^{i k_{i}}\right) \\
& \times \operatorname{tr}\left[E^{x_{1}-1} A_{k_{i}} E A_{k_{j}} E^{x_{3}-x_{1}-1} A_{k_{l}} E^{N-x_{3}+1} \Omega_{P}\right]= \\
& \sum_{i, j, l=1}^{3}\left(c_{3} b_{2} \phi_{1}^{i} \phi_{1}^{j} \phi_{1}^{l}-c_{3} c_{1} \phi_{1}^{i} \phi_{1}^{j} \phi_{2}^{l}\right) \operatorname{tr}\left[E^{x_{2}-1} B_{k_{i}, k_{j}} E^{x_{3}-x_{2}} A_{k_{j}} E^{N-x_{3}+1} \Omega_{P}\right] .
\end{aligned}
$$

As relações provenientes da configuração apresentada na figura 4.7 são análogas às 
relações advindas da configuração da figura 4.6. Consideremos agora as relações provenientes da configuração mostrada na figura 4.8

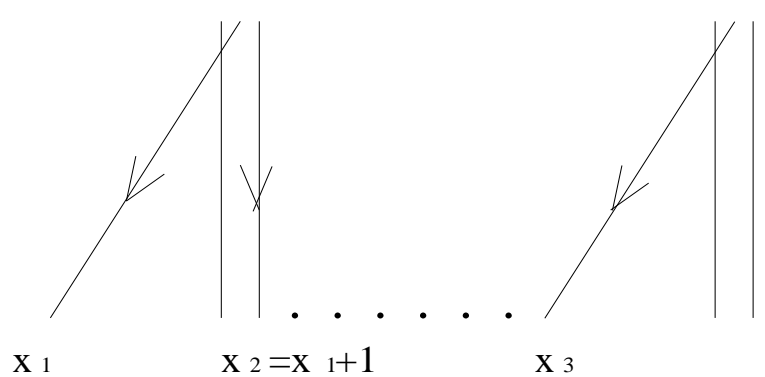

Figura 4.7: Configuração de 2 flechas juntas e uma separada (situação 4).

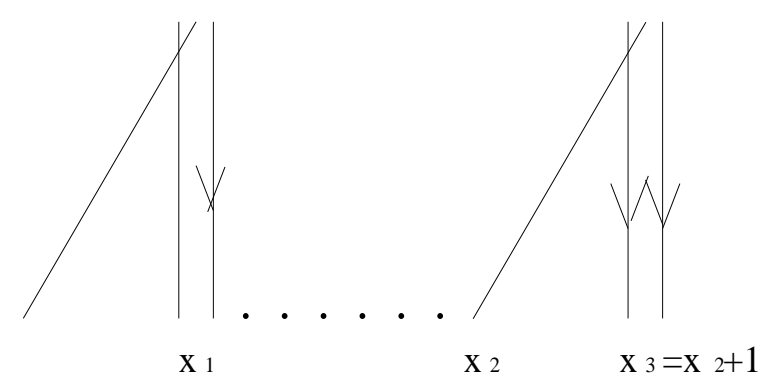

Figura 4.8: Configuração de 2 flechas juntas e uma separada (situação 5).

As equações das amplitudes correspondentes neste caso são escritas como

$$
\begin{aligned}
& \Lambda_{3} \phi_{111}^{2 * 3}\left(x_{1}, x_{2}, x_{2}\right)=b_{2} b_{3} \phi_{111}^{2 * 3}\left(x_{1}, x_{2}, x_{3}\right)+b_{2} a_{2} \phi_{112}\left(x_{1}, x_{2}, x_{2}\right)+ \\
& c_{1} b_{3} \phi_{211}^{2 * 3}\left(x_{1}, x_{2}, x_{2}\right)+c_{1} a_{2} \phi_{212}\left(x_{1}, x_{2}, x_{2}\right) .
\end{aligned}
$$

Substituindo-se o ansatz matricial (4.3) e (4.4) juntamente com (4.6) nesta última equação e utilizando as relações de comutação (4.7) obtemos depois de algumas simplificações 


$$
\begin{aligned}
& \sum_{i, j, l=1}^{3} \operatorname{tr}\left[E^{x_{1}-1} A_{k_{i}} E E^{x_{2}-x_{1}-1} A_{k_{j}, k_{l}} E^{N-x_{2}} \Omega_{P}\right]\left(\Lambda_{3} \phi_{1}^{i}-b_{2} b_{3} \phi_{1}^{i}-b_{2} a_{2} \phi_{2}^{i}\right) \phi_{1}^{j} \phi_{1}^{l}= \\
& \sum_{i, j, l=1}^{3} \operatorname{tr}\left[E^{x_{1}-1} A_{k_{i}} E E^{x_{2}-x_{1}-1} A_{k_{j}} E E^{-1} A_{k_{l}} E E^{N-x_{2}} \Omega_{P}\right]\left(b_{2} a_{2} \phi_{1}^{i} \phi_{1}^{j} \phi_{2}^{l}+c_{1} a_{2} \phi_{2}^{i} \phi_{1}^{j} \phi_{2}^{l}\right) .
\end{aligned}
$$

As relações advindas da configuração da figura 4.9 são semelhantes a esta última. Consideremos agora a configuração mostrada na figura 4.10.

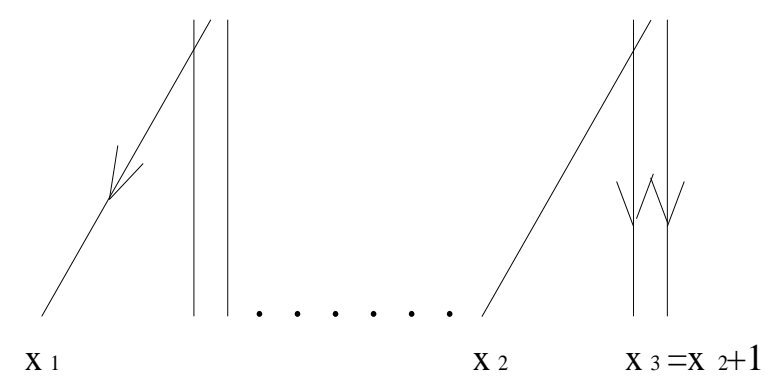

Figura 4.9: Configuração de 2 flechas juntas e uma separada (situação 6).

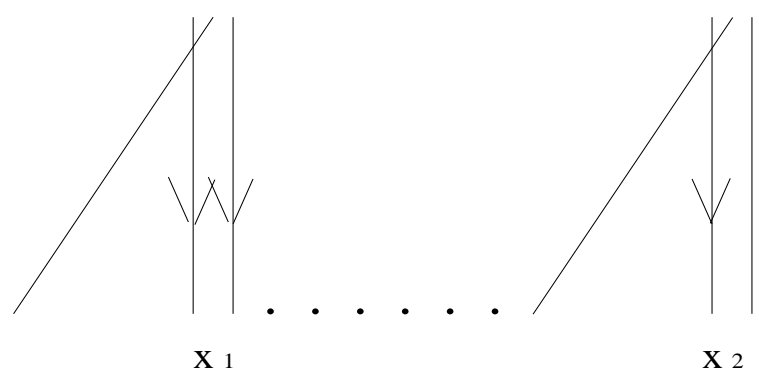

Figura 4.10: Configuração de 2 flechas juntas e uma separada (situação 7).

As equações das amplitudes neste caso são escritas como

$$
\begin{aligned}
& \Lambda_{3} \phi_{111}^{1 * 2}\left(x_{1}, x_{1}, x_{2}\right)=a_{2} b_{2} \phi_{121}\left(x_{1}, x_{1}, x_{2}\right)+b_{3} b_{2} \phi_{111}^{1 * 2}\left(x_{1}, x_{1}, x_{2}\right) \\
& +a_{2} c_{1} \phi_{122}\left(x_{1}, x_{1}, x_{2}\right)+b_{3} c_{1} \phi_{112}^{1 * 2}\left(x_{1}, x_{1}, x_{2}\right) .
\end{aligned}
$$


Substituindo-se as relações (4.3) e (4.4), juntamente com (4.6) e usando-se (4.7) obtemos

$$
\begin{aligned}
& \sum_{i, j, l=1}^{3} \phi_{1}^{i} \phi_{1}^{j}\left(\Lambda_{3} \phi_{1}^{l}-b_{3} b_{2} \phi_{1}^{l}-b_{3} c_{1} \phi_{2}^{l}\right) \operatorname{tr}\left[E^{x_{1}-1} B_{k_{i}, k_{j}} E^{x_{2}-x_{1}} A_{k_{l}} E^{N-x_{2}+1} \Omega_{P}\right]= \\
& \sum_{i, j, l=1}^{3}\left(a_{2} b_{2} \phi_{1}^{i} \phi_{2} \phi_{1}^{l}+a_{2} c_{1} \phi_{1}^{i} \phi_{2}^{j} \phi_{2}^{l}\right) \operatorname{tr}\left[E^{x_{1}-1} A_{k_{i}} A_{k_{j}} E^{x_{2}-x_{1}} A_{k_{l}} E^{N-x_{2}+1}\right] .
\end{aligned}
$$

As relações oriundas das amplitudes exibidas na figura 4.11 são análogas àquelas derivadas anteriormente. Desta forma encerramos parcialmente o estudo das situações em que duas das três flechas estão em posição de colisão, e a terceira esteja separada destas duas.

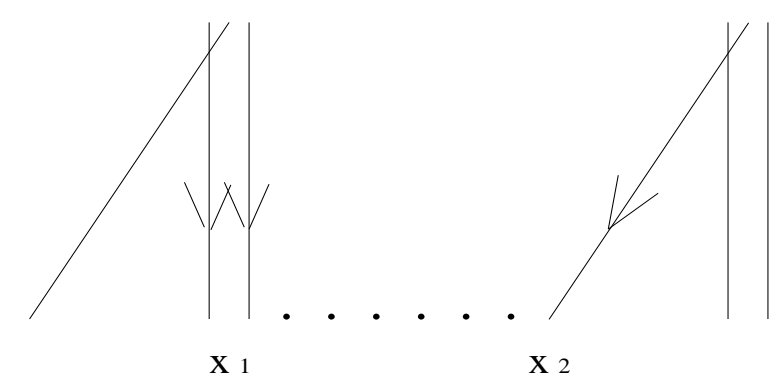

Figura 4.11: Configuração de 2 flechas juntas e uma separada (situação 8).

A última equação referente a este problema é dada quando as tres flechas estão juntas. Mostramos na figura 4.12 a representação envolvendo a colisão das três flechas. As equações das amplitudes neste caso são

$$
\Lambda_{3} \phi_{211}^{2 * 3}\left(x_{1}, x_{2}, x_{2}\right)=a_{3} \phi_{112}^{1 * 2}\left(x_{2}, x_{2}, x_{2}\right) .
$$

Substituindo o ansatz (4.3),(4.4) e (4.6) nesta última equação, obtemos 


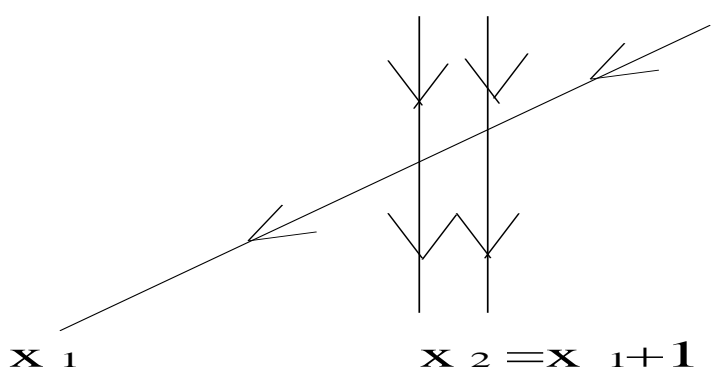

Figura 4.12: Configuração de 3 flechas juntas.

$$
\begin{aligned}
& \Lambda_{3} \sum_{i, j, l=1}^{3} \operatorname{tr}\left[E^{x_{1}-1} \phi_{2}^{i} A_{k_{i}} E^{x_{2}-x_{1}} \phi_{1}^{j} \phi_{1}^{l} A_{k_{j}, k_{l}} E^{N-x_{2}+1} \Omega_{P}\right]= \\
& a_{3} \sum_{i, j, l=1}^{3} \operatorname{tr}\left[E^{x_{2}-1} \phi_{1}^{i} \phi_{1}^{j} B_{k_{i}, k_{j}} \phi_{2}^{l} A_{k_{l}} E^{N-x_{2}+1} \Omega_{P}\right] .
\end{aligned}
$$

Assim as equações (4.23),(4.25), (4.27), (4.29) e (4.31) devem ser simultâneamente satisfeitas para que o nosso ansatz do produto matricial seja válido. Contudo é simples verificar que as relações (4.23), (4.25), (4.27) e (4.29) advindas das configurações onde apenas duas flechas estão em colisão decorrem de duas relações, semelhantes àquelas obtidas quando estudamos o setor com apenas duas flechas. Para isto consideremos inicialmente a relação (4.23). Substituindo $\Lambda_{3}=\Lambda\left(k_{i}\right) \Lambda\left(k_{j}\right) \Lambda\left(k_{l}\right)$ e a relação $\Lambda\left(k_{i}\right) \phi_{1}^{i}=c_{1} \phi_{2}^{i}+b_{2} \phi_{1}^{i}(i=1,2,3)$ obtida de (4.15), em (4.29) obtemos

$$
\begin{aligned}
& \sum_{i, j, l=3}^{3} \operatorname{tr}\left[E^{x_{1}-1} A_{k_{i}} E^{x_{2}-x_{1}} A_{k_{j}} E^{x_{2}-1-x_{2}-1} E A_{k_{l}} E^{N-x_{3}+1} \Omega_{P}\right] \\
& \times e^{i k_{l}}\left(\Lambda\left(k_{j}\right) \Lambda\left(k_{l}\right) c_{1} \phi_{2}^{i} \phi_{2}^{j} \phi_{1}^{l}+\Lambda\left(k_{j}\right) \Lambda\left(k_{l}\right) b_{2} \phi_{1}^{i} \phi_{2}^{j} \phi_{1}^{l}-b_{2} a_{1} \phi_{1}^{i} \phi_{1}^{j} \phi_{2}^{l} e^{i k_{j}}\right. \\
& \left.-c_{1} a_{1} \phi_{2}^{i} \phi_{1}^{j} \phi_{2}^{l} e^{i k_{j}}\right)=\sum_{i, j, l=1}^{3} \operatorname{tr}\left[E^{x_{1}-1} A_{k_{i}} E^{x_{2}-x_{1}+1} B_{k_{j}, k_{l}} E^{N-x_{3}+1} \Omega_{P}\right] \\
& \times\left(b_{2} c_{3} \phi 1^{i} \phi_{1}^{j} \phi_{1}^{l}+c_{1} c_{3} \phi_{2}^{i} \phi_{1}^{j} \phi_{1}^{l}\right) e^{i\left(k_{j}+k_{l}\right)} .
\end{aligned}
$$

Esta relação pode ser colocada na seguinte forma 


$$
\begin{aligned}
& \sum_{i=1}^{3} \phi_{i}^{i} b_{2}\left\{\sum_{j, l=1}^{3}\left(\Lambda\left(k_{j}\right) \Lambda\left(k_{l}\right) \phi_{2}^{j} \phi_{1}^{l} e^{i k_{l}}-a_{1} \phi_{1}^{j} \phi_{2}^{l} e^{i\left(k_{j}+k_{l}\right)}\right)\right. \\
& \times \operatorname{tr}\left[E^{x_{1}-1} A_{k_{i}} E^{x_{2}-x_{1}} A_{k_{j}} A_{k_{l}} E^{N-x_{3}+2} \Omega_{P}\right]- \\
& \left.\sum_{j, l=1}^{3} c_{3} \phi_{1}^{j} \phi_{2}^{l} e^{i\left(k_{j}+k_{l}\right)} \operatorname{tr}\left[E^{x_{1}-1} A_{k_{i}} E^{x_{2}-x_{1}} B_{k_{j}, k_{l}} E^{N-x_{3}+2} \Omega_{P}\right]\right\}= \\
& \sum_{i=1}^{3} \phi_{2}^{i} c_{1}\left\{\sum_{j, l=1}^{3}\left(\Lambda\left(k_{j}\right) \Lambda\left(k_{l}\right) \phi_{2}^{j} \phi_{1}^{l} e^{i k_{l}}-a_{1} \phi_{1}^{j} \phi_{2}^{l} e^{i\left(k_{j}+k_{l}\right)}\right)\right. \\
& \times \operatorname{tr}\left[E^{x_{1}-1} A_{k_{i}} E^{x_{2}-x_{1}} A_{k_{j}} A_{k_{l}} E^{N-x_{3}+2} \Omega_{P}\right] \\
& \left.\sum_{j, l=1}^{3} c_{3} \phi_{1}^{j} \phi_{1}^{l} e^{i\left(k_{j}+k_{l}\right)} \operatorname{tr}\left[E^{x_{1}-1} A_{k_{i}} E^{x_{2}-x_{1}} B_{k_{j}, k_{l}} E^{N-x_{3}+2} \Omega_{P}\right]\right\},
\end{aligned}
$$

que é satisfeita caso, para cada $i=1,2,3$,

$$
\begin{aligned}
& \sum_{j, l=1}^{3}\left(\Lambda\left(k_{j}\right) \Lambda\left(k_{l}\right) \phi_{2}^{j} \phi_{1}^{l} e^{i k_{l}}-a_{1} \phi_{1}^{j} \phi_{2}^{l} e^{i\left(k_{j}+k_{l}\right)}\right) \\
& \times \operatorname{tr}\left[E^{x_{1}-1} A_{k_{i}} E^{x_{2}-x_{1}} A_{k_{j}} A_{k_{l}} E^{N-x_{3}+2} \Omega_{P}\right]- \\
& \sum_{j, l=1}^{3} c_{3} \phi_{1}^{j} \phi_{2}^{l} e^{i\left(k_{j}+k_{l}\right)} \operatorname{tr}\left[E^{x_{1}-1} A_{k_{i}} E^{x_{2}-x_{1}} B_{k_{j}, k_{l}} E^{N-x_{3}+2} \Omega_{P}\right]=0 .
\end{aligned}
$$

Repare que conforme já mencionado, a relação acima é uma mera generalização da equação (4.12) obtida no setor de duas flechas. Por procedimento análogo é simples verificar que as equações (4.25) também são satisfeitas caso a equação (4.34) também o seja.

De forma análoga, as equações (4.27) e (4.29) são satisfeitas simultâneamente, pois tais equações constituem uma generalização da equação (4.13).

Quando relacionamos a equação (4.23) com (4.29) ou (4.25) com (4.27) mediante a eliminação dos traços envolvendo a matriz $B_{k_{i}, k_{j}}$, encontramos de forma similar ao ocorrido no setor com duas flechas, as relações que fixam a álgebra a ser obedecida pelas matrizes $A_{k_{i}}(i=1,2,3)$; i.é., 


$$
\begin{aligned}
& A_{k_{i}} A_{k_{j}}=-\frac{\alpha\left(k_{j}, k_{i}\right)}{\alpha\left(k_{i}, k_{j}\right)} A_{k_{j}} A_{k_{i}}, \quad A_{k_{i}}^{2}=0, \\
& \alpha\left(k_{j}, k_{i}\right)= \\
& {\left[\left(\Lambda\left(k_{j}\right) b_{1}+q_{1}\right) \Lambda\left(k_{j}\right)-a_{1}\left(\Lambda\left(k_{j}\right)-b_{2}\right)\right]\left[\left(\Lambda\left(k_{i}\right) \Lambda\left(k_{j}\right)-b_{3}\right)-a_{2} c_{3}\left(\Lambda\left(k_{j}\right)-b_{2}\right)\right]}
\end{aligned}
$$

Por fim vamos analisar a equação (4.31) proveniente da configuração onde as três flechas estâo em posição de colisão. Escrevendo-se $\phi_{2}^{i}$ e $\phi_{2}^{l}$ em termos de $\phi_{1}^{i}$ e $\phi_{1}^{l}$ respectivamente (veja (4.15)), esta equação pode ser escrita como

$$
\begin{aligned}
& \Lambda_{3} \sum_{i, j, l=3}^{3} e^{-i k_{i}}\left(\Lambda\left(k_{i}\right)-b_{2}\right) \operatorname{tr}\left[E^{x_{1}} A_{k_{i}} B_{k_{j}, k_{l}} E^{N-x_{2}+1} \Omega_{P}\right]= \\
& a_{3} \sum_{i, j, l=1}^{3}\left(\Lambda\left(k_{l}\right)-b_{2}\right) \operatorname{tr}\left[E^{x_{1}} B_{k_{i}, k_{j}} A_{k_{l}} E^{N-x_{2}+1} \Omega_{P}\right] .
\end{aligned}
$$

Denotando-se

$$
\begin{aligned}
& P_{1}(\Lambda(k))=\Lambda(k)\left(\Lambda(k)-b_{2}\right) e^{-i k}-b_{1} \Lambda_{p}+q_{1}, \\
& P_{2}(\Lambda(k))=\Lambda(k)-b_{2},
\end{aligned}
$$

e lembrando que $\Lambda_{3}=\Lambda\left(k_{1}\right) \Lambda\left(k_{2}\right) \Lambda\left(k_{3}\right)$, a equação (4.36) tem a forma simplificada

$$
\begin{aligned}
& \sum_{i=1} \Lambda\left(k_{j}\right) \Lambda\left(k_{l}\right) P_{1}\left(\Lambda\left(k_{i}\right)\right) \sum_{j, l=1} \operatorname{tr}\left[E^{x_{1}} A_{k_{i}} B_{k_{j}, k_{l}} E^{N-x_{2}+1} \Omega_{P}\right]= \\
& a_{3} \sum_{l=1} P_{2}\left(\Lambda\left(k_{l}\right)\right) \sum_{i, j=1} \operatorname{tr}\left[E^{x_{1}} B_{k_{i}, k_{j}} A_{k_{l}} E^{N-x_{2}+1} \Omega_{P}\right] .
\end{aligned}
$$

Entretanto da equação (4.12) extraímos a propriedade algébrica

$$
c_{3} c_{1} \sum_{j, l=1} A_{k_{j}, k_{l}}=\sum_{j, l=1} A_{k_{j}} A_{k_{l}}\left[\Lambda\left(k_{l}\right) P_{1}\left(\Lambda\left(k_{j}\right)\right)-a_{1} P_{2}\left(\Lambda\left(k_{l}\right)\right)\right], \quad j \neq l .
$$


Usando esta última relação em (4.38), obteremos após multiplicarmos por $c_{1} c_{3}$

$$
\begin{aligned}
& \sum_{i=1} \Lambda\left(k_{j}\right) \Lambda\left(k_{l}\right) P_{1}\left(\Lambda\left(k_{i}\right)\right) \sum_{j, l=1}^{3}\left[\Lambda\left(k_{l}\right) P_{1}\left(\Lambda\left(k_{j}\right)\right)-a_{1} P_{2}\left(\Lambda\left(k_{l}\right)\right)\right] \\
& \operatorname{tr}\left[E^{x_{1}} A_{k_{i}} A_{k_{j}} A_{k_{l}} E^{N-x_{2}+1} \Omega_{P}\right]= \\
& a_{3} \sum_{l=1} P_{2} \Lambda\left(k_{l}\right) \sum_{i, j=1}\left[\Lambda\left(k_{j}\right) P_{1}\left(\Lambda\left(k_{i}\right)\right)-a_{1} P_{2}\left(\Lambda\left(k_{j}\right)\right)\right] \operatorname{tr}\left[E^{x_{1}} A_{k_{i}} A_{k_{j}} A_{k_{l}} E^{N-x_{2}+1} \Omega_{P}\right] .
\end{aligned}
$$

É conveniente, para prosseguirmos, introduzir a notação

$$
N(z, w)=z P_{1}(w)-a_{1} P_{2}(z)
$$

$$
\begin{aligned}
& F\left(k_{i}, k_{j}, k_{l}\right)=\Lambda\left(k_{j}\right) \Lambda\left(k_{l}\right) P_{1}\left(\Lambda\left(k_{i}\right)\right) N\left(\Lambda\left(k_{i}\right), \Lambda\left(k_{j}\right)\right)-a_{3} P_{2}\left(\Lambda\left(k_{j}\right)\right) N\left(\Lambda\left(k_{j}\right), \Lambda\left(k_{i}\right)\right), \\
& H_{i, j, l}=\operatorname{tr}\left[E^{x_{1}} A_{k_{i}} A_{k_{j}} A_{k_{l}} E^{N-x_{2}+1} \Omega_{P}\right] .
\end{aligned}
$$

A equação (4.40) torna-se então

$$
\begin{aligned}
& H_{1,2,3} F(1,2,3)+H_{1,3,2} F(1,3,2)+H_{2,1,3} F(2,1,3) \\
& +H_{2,3,1} F(2,3,1)+H_{3,1,2} F(3,1,2)+H_{3,1,2} F(3,1,2)=0 .
\end{aligned}
$$

Vamos utilizar a relação de comutação (4.35) entre as matrizes $A_{k_{i}}, A_{k_{j}}$ e $A_{k_{l}}$ para reescrevermos a última expressão em termos de $H_{1,2,3}$, ou seja, 


$$
\begin{aligned}
& A_{k_{1}} A_{k_{3}} A_{k_{2}}=-\frac{\alpha_{2,3}}{\alpha_{3,2}} A_{k_{1}} A_{k_{2}} A_{k_{3}}, \\
& A_{k_{2}} A_{k_{1}} A_{k_{3}}=-\frac{\alpha_{1,2}}{\alpha_{2,1}} A_{k_{1}} A_{k_{2}} A_{k_{3}}, \\
& A_{k_{2}} A_{k_{3}} A_{k_{1}}=\frac{\alpha_{1,3}}{\alpha_{3,1}} \frac{\alpha_{1,2}}{\alpha_{2,1}} A_{k_{1}} A_{k_{2}} A_{k_{3}}, \\
& A_{k_{3}} A_{k_{1}} A_{k_{2}}=\frac{\alpha_{1,3}}{\alpha_{3,1}} \frac{\alpha_{2,3}}{\alpha_{3,2}} A_{k_{1}} A_{k_{2}} A_{k_{3}}, \\
& A_{k_{3}} A_{k_{2}} A_{k_{1}}=-\frac{\alpha_{2,3}}{\alpha_{3,2}} \frac{\alpha_{1,3}}{\alpha_{3,1}} \frac{\alpha_{1,2}}{\alpha_{2,1}} A_{k_{1}} A_{k_{2}} A_{k_{3}}
\end{aligned}
$$

Denotando $\Lambda\left(k_{1}\right)$ por $x, \Lambda\left(k_{2}\right)$ por $y$ e $\Lambda\left(k_{3}\right)$ por $z$, a expressão (4.43) pode ser reescrita como

$$
\begin{aligned}
& F(x, y, z) \alpha(z, y) \alpha(z, x) \alpha(y, x)-F(x, z, y) \alpha(y, z) \alpha(z, x) \alpha(y, x) \\
& -F(y, x, z) \alpha(x, y) \alpha(z, y) \alpha(z, x)+F(y, z, x) \alpha(x, z) \alpha(x, y) \alpha(z, y) \\
& +F(z, x, y) \alpha(x, z) \alpha(y, z) \alpha(y, x)-F(z, y, x) \alpha(y, z) \alpha(x, z) \alpha(x, y)=0 .(4.4
\end{aligned}
$$

Para melhor visualizarmos a expressão (4.45) é conveniente relembrarmos as funções já definidas:

$$
\begin{aligned}
& F(x, y, z)=y z P_{1}(x) N(z, y)-a_{3} P_{2}(z) N(y, z), \\
& N(x, y)=x P_{1}(y)-a_{1} P_{2}(x), \\
& P_{1}(x)=b_{1} x+q_{1}, \\
& P_{2}(x)=x-b_{2}, \\
& \alpha(x, y)=\left[q_{2}\left(b_{2}-y\right)-\left(b_{3}-x y\right)\right]\left[a_{1}\left(b_{2}-y\right)+\left(q_{1}+b_{1} x\right) y\right] .
\end{aligned}
$$

As expressões (4.46) nos dizem que (4.45) pode ser escrita como

$$
\sum_{l, m, n=0}^{7} A_{l, m, n} x^{l} y^{m} z^{n}=0
$$


sendo $A_{l, m, n}$ funções dos parâmetros $a_{1}, a_{3}, b_{1}, b_{2}, b_{1}, q_{1}$ e $q_{2}$. Para termos uma variedade exatamente integrável no espaço dos parâmetros acima precisamos impor que (4.47) seja verdadeira para quaisquer $x, y$ e $z$, pois não queremos obter vínculos para $k_{1}, k_{2}$ e $k_{3}$ neste estágio. ${ }^{2}$

Antes de buscarmos as possíveis variedades exatamente integráveis vamos considerar o caso em que $n$ é geral.

\section{- Setor com $n$ flechas}

As autofunções deste setor são dadas por (4.2), sendo as amplitudes dadas pelo ansatz matricial (4.3) e (4.4). A equação de autovalor imporá relações entre as amplitudes que para serem satisfeitas fixará a álgebra das matrizes $A^{(\alpha)}(\alpha=1,2,3)$ e $E$. As relações advindas das configurações $\mid x_{1}, \alpha_{1} ; \ldots ; x_{n}, \alpha_{n}>$ onde todas as flechas não estão em posições de colisão, implicam na identificação

$$
A^{(\alpha)}=\sum_{i=1}^{n} \phi_{\alpha}^{i} A_{k_{i}} E, \quad(\alpha=1,2), \quad A^{(3)}=\sum_{i, j=1, i \neq j}^{n} \phi_{1}^{i} \phi_{1}^{j} B_{k_{i}, k_{j}} E
$$

sendo que as matrizes $\left\{A_{k_{i}}\right\}$ e $\left\{B_{k_{j}, k_{l}}\right\}$, satisfazem às relações de comutação

$$
\begin{aligned}
& E A_{k_{i}}=e^{i k_{i}} A_{k_{i}} E, \quad A_{k_{i}} \Omega_{P}=\Omega_{P} A_{k_{i}} \quad(i=1, \ldots, n), \\
& E B_{k_{j}, k_{l}}=e^{i\left(k_{j}+k_{l}\right)} B_{k_{j}, k_{l}} E, \quad B_{k_{j}, k_{l}} \Omega_{P}=\Omega_{P} B_{k_{j}, k_{l}} \quad(j, l=1, \ldots, n) \quad j \neq l .
\end{aligned}
$$

Mais ainda, os autovalores $\Lambda_{n}$ são dados pelos produtos dos autovalores do caso de uma flecha, isto é,

$$
\Lambda_{n}^{l_{1}, l_{2}, \ldots, l_{n}}\left(k_{1}, k_{2}, \ldots, k_{n}\right)=\Lambda_{1}^{l_{1}}\left(k_{1}\right) \Lambda_{1}^{l_{2}}\left(k_{2}\right) \cdots \Lambda_{1}^{l_{n}}\left(k_{n}\right),
$$

\footnotetext{
${ }^{2}$ Tais parâmetros espectrais serão fixos pelas relações advindas da consistência da álgebra com a propriedade cíclica do traço (análoga às relações advindas das condições de contorno na formulação do ansatz de Bethe).
} 
sendo $\Lambda_{1}^{l_{j}}\left(k_{j}\right)$ dados por (3.20). Como no problema de 5 vértices interagentes, o momento neste setor em termos da variav́eis $\left\{k_{j}\right\}$ nos fornece a relação

$$
P=\sum_{l=1}^{n} k_{l} .
$$

As equações obtidas das configurações em que apenas duas das flechas estão juntas (colisão) são simples generalizações das equações (4.35), isto é:

$$
\begin{aligned}
& A_{k_{i}} A_{k_{j}}=-\frac{\alpha\left(k_{j}, k_{i}\right)}{\alpha\left(k_{i}, k_{j}\right)} A_{k_{j}} A_{k_{i}}, \quad A_{k_{i}}^{2}=0 \quad i, j=1, \ldots, n \\
& \alpha(i, j)= \\
& {\left[\left(\Lambda\left(k_{j}\right) b_{1}+q_{1}\right) \Lambda\left(k_{j}\right)-a_{1}\left(\Lambda\left(k_{j}\right)-b_{2}\right)\right]\left[\left(\Lambda\left(k_{i}\right) \Lambda\left(k_{j}\right)-b_{3}\right)-a_{2} c_{3}\left(\Lambda\left(k_{j}\right)-b_{2}\right)\right] .}
\end{aligned}
$$

As relações advindas das configurações em que 3 das $n$ flechas estão em colisão nos fornecem relações que são generalizações de (4.47), isto é,

$$
\sum_{P} H_{p_{1}, p_{2}, p_{3}} F\left(k_{p_{1}}, k_{p_{2}}, k_{p_{3}}\right)=0
$$

sendo que a soma é sobre as permutações não repetidas de três índices $\left(p_{1}, p_{2}, p_{3}\right)$ distintos no conjunto $(1,2, \ldots, n)$. Não é imediato verificar, mas um cálculo longo e tedioso nos mostra que as configurações com 4 ou mais flechas em colisão fornecerão relações que são satisfeitas caso as relações (4.8) já o sejam. Por fim, para completar o cálculo das amplitudes precisamos calcular os valores dos parâmetros espectrais $\left(k_{1}, \ldots, k_{n}\right)$, até aqui indeterminado. Para isto usaremos a propriedade cíclica do traço na definição do ansatz matricial para uma componente qualquer, por exemplo, 


$$
\begin{aligned}
& \operatorname{Tr}\left[A_{k_{1}} \cdots A_{k_{j}} \cdots A_{k_{n}} E^{N} \Omega_{P}\right]= \\
& \prod_{l=j+1}^{n} \frac{\alpha\left(k_{l}, k_{j}\right)}{\alpha\left(k_{j}, k_{l}\right)} \operatorname{Tr}\left[A_{k_{1}} \cdots A_{k_{j-1}} A_{k_{j+1}} \cdots A_{k_{n}} A_{k_{j}} E^{N} \Omega_{P}\right]= \\
& e^{-i k_{j} N} \prod_{l=1(l \neq j)}^{n} \frac{\alpha\left(k_{l}, k_{j}\right)}{\alpha\left(k_{j}, k_{l}\right)} \operatorname{Tr}\left[A_{k_{1}} \cdots A_{k_{j}} \ldots A_{k_{n}} E^{N} \Omega_{P}\right],
\end{aligned}
$$

onde usamos as relações de comutação (4.49) e repetidas vezes (4.52). Assim, as equações não lineares que fixam os parâmetros espectrais são

$$
e^{i k_{j} N}=-\prod_{l=1}^{n} \frac{\alpha\left(k_{l}, k_{j}\right)}{\alpha\left(k_{j}, k_{l}\right)}, \quad j=1, \ldots, n
$$

sendo $\alpha\left(k_{l}, k_{j}\right)$ dado por (4.52). Estas equações são análogas às obtidas pelo ansatz de Bethe [32].

\subsection{Análise das soluções}

O problema de se encontrar modelos de 10 vértices exatamente integráveis se resume em achar no espaço de 7 parâmetros $\left(a_{1}, a_{3}, b_{1}, b_{2}, b_{3}, q_{1}, q_{2}\right)$ as possíveis soluções de (4.47). A obtenção dos coeficiente $A_{l, m, n}$ em (4.47) não é simples de se fazer analíticamente. Utilizamos para tal fim o software Mathematica [31]. Encontramos três ramos de possíveis soluções de (4.47) que passaremos a discutir

Caso 1: $q^{2}=0$

Neste caso $c_{3}$ ou $a_{2}$ devem ser nulos. Isto implica necessariamente, como consequência das equações a serem satisfeitas, que as possíveis configurações da rede envolvendo 4 dos pesos, isto é, $a_{2}, a_{3}, b_{3}$ e $c_{3}$ não serão contabilizadas na função de partição, e deste modo, só podemos construir configurações para a rede envolvendo seis dos vértices. Vértices estes que coincidem com aqueles do modelo de seis vértices discutido no capítulo 3 . 
Caso 2:

Neste caso três dos parâmetros são fixos em termos dos outro quatro, i. é.,

$$
\begin{aligned}
q_{2} & =\frac{a_{1} b_{2}\left(a_{1}-q_{1}\right)}{b_{1}\left(a_{1}+b_{1} b_{2}\right)}\left(b_{1} b_{2}+q_{1}\right), \\
a_{3} & =\frac{a_{1}^{2}\left(a_{1}-q_{1}\right)}{b_{1}\left(a_{1}+b_{1} b_{2}\right)}, \\
b_{3} & =\frac{b_{2}^{2}\left(a_{1}-q_{1}\right)}{a_{1}+b_{1} b_{2}} .
\end{aligned}
$$

É conhecida na literatura uma solução exata para um modelo especial de 10 vértices. Esta solução foi obtida por de Vega e Woynarovich [20] utilizando-se o método de fusão, método este apropriado para as matrizes de transferência linha-para-linha. Este modelo tem as fugacidades simetrizadas e são dadas em termos de dois parâmet$\operatorname{ros}(\theta$ e $\gamma)$

$$
\begin{aligned}
& a_{0}=a_{3}=1, \\
& c_{1}=c_{2}=c_{3}=\frac{\sinh (\gamma) \sqrt{(2 \cosh (\gamma))}}{\sinh \left(\theta+\frac{3 \gamma}{2}\right)}, \\
& b_{1}=b_{3}=\frac{\sinh \left(\theta-\frac{\gamma}{2}\right)}{\sinh \left(\theta+\frac{3 \gamma}{2}\right)} \\
& b_{2}=a_{1}=\frac{\sinh \left(\theta+\frac{\gamma}{2}\right)}{\sinh \left(\theta+\frac{3 \gamma}{2}\right)}
\end{aligned}
$$

A simples substituição nos mostra que a solução (4.57) está contida em (4.56). Repare que ao invés de 2 parâmetros livres, como em (4.57), temos em (4.56) 4 parâmetros livres. Uma interessante continuação ao nosso trabalho seria um estudo mais abrangente das propriedades físicas correspondentes à nossa solução geral (4.56). Substituindo-se os vínculos (4.56) na equação (4.55) e escrevendo-se $e^{i k_{j}}$ em termos de $\Lambda\left(k_{j}\right)$ (veja (3.43)), obtemos a forma final para as equações que fixam o conjunto $\left(k_{1}, k_{2}, \ldots, k_{n}\right)$ 


$$
\begin{aligned}
& {\left[\frac{\Lambda\left(k_{j}\right)\left(\Lambda\left(k_{j}\right)-b_{2}\right)}{\Lambda\left(k_{j}\right) b_{1}+q_{1}}\right]^{N}=} \\
& (-1)^{n+1} \prod_{l=1}^{n} \frac{b_{1}\left(a_{1}+b_{1} b_{2}\right) \Lambda\left(k_{j}\right) \Lambda\left(k_{l}\right)-\left(a_{1}-q_{1}\right)\left(a_{1}+b_{1} b_{2}\right) \Lambda\left(k_{j}\right)+a_{1} b_{2}\left(a_{1}-q_{1}\right)}{b_{1}\left(a_{1}+b_{1} b_{2}\right) \Lambda\left(k_{j}\right) \Lambda\left(k_{l}\right)-\left(a_{1}-q_{1}\right)\left(a_{1}+b_{1} b_{2}\right) \Lambda\left(k_{j}\right)+a_{1} b_{2}\left(a_{1}-q_{1}\right)} .
\end{aligned}
$$

Caso 3:

Neste caso, assim como no anterior, três dos vínculos são fixos:

$$
\begin{aligned}
& q_{2}=\frac{\left(q_{1}-a_{1}\right)}{b_{1}^{2}}\left(a_{1}^{2}+q_{1}^{2}-a_{1}\left(b_{1} b_{2}+2 q_{1}\right)\right), \\
& a_{3}=\frac{\left(a_{1}-q_{1}\right)}{b_{1}} q_{1}, \\
& b_{3}=\frac{\left(a_{1}-q_{1}\right)}{b_{1}^{2}}\left(q_{1}+b_{1} b_{2}-a_{1}\right) .
\end{aligned}
$$

Substituindo-se estes vínculos na equação (4.55), como no caso anterior, obtemos

$$
\left[\frac{\Lambda\left(k_{j}\right)\left(\Lambda\left(k_{j}\right)-b_{2}\right)}{\Lambda\left(k_{j}\right) b_{1}+q_{1}}\right]^{N}=(-1)^{n+1} \prod_{l=1}^{n} \frac{\Lambda\left(k_{j}\right) \Lambda\left(k_{l}\right)-\frac{q_{1}-a_{1}}{b_{1}} \Lambda\left(k_{j}\right)+\left(\frac{q_{1}-a_{1}}{b_{1}}\right)^{2}}{\Lambda\left(k_{j}\right) \Lambda\left(k_{l}\right)-\frac{q_{1}-a_{1}}{b_{1}} \Lambda\left(k_{l}\right)+\left(\frac{q_{1}-a_{1}}{b_{1}}\right)^{2}}
$$

Dividindo-se o numerador e o denominador de ambos os lados desta última equação por $\frac{q_{1}-a_{1}}{b_{1}}$, e definindo-se

$$
\lambda_{i}=\frac{\Lambda\left(k_{i}\right)}{\frac{q_{1}-a_{1}}{b_{1}}},
$$

obtemos

$$
\left[\frac{\lambda_{j}\left(\lambda_{j}-\frac{b_{1} b_{2}}{q_{1}-a_{1}}\right)}{\frac{b_{1}^{2}}{q_{1}-a_{1}}\left(\lambda_{j}+\frac{q_{1}}{q_{1}-a_{1}}\right)}\right]^{N}=(-1)^{n+1} \prod_{l=1}^{n} \frac{\lambda_{j} \lambda_{l}-\lambda_{j}+1}{\lambda_{j} \lambda_{l}-\lambda_{l}+1} \quad j=1, \cdots, n .
$$

Estas últimas equações juntamente com a equação (4.50) determinam o espectro de um novo modelo de 10 vértices. Comparando-se o lado direito das equações (4.62) 
e (3.65) vemos que os mesmos coincidem para o caso em que temos no modelo de 6 vértices $\Delta=\frac{1}{2}$. Assim o caso 3 pode ser pensado como uma deformação do modelo de 6 vértices simétrico com anisotropia $\Delta=\frac{1}{2}$. Para melhor elucidarmos tal fato consideraremos a versão simetrizada de (4.59) em que $b_{1}=b_{2}=b$ e $c_{1}=c_{2}=c$. Neste caso as equações que fixam os parâmetros espectrais são

$\left[\frac{\lambda_{j}\left(\lambda_{j}-\frac{b^{2}}{c^{2}-a_{1}-b^{2}}\right)}{\frac{b^{2}}{c^{2}-a_{1}-b^{2}}\left(\lambda_{j}-\frac{b^{2}}{c^{2}-a_{1}-b^{2}}\right)+\frac{(b c)^{2}}{\left(c^{2}-a_{1}-b^{2}\right)^{2}}}\right]^{N}=(-1)^{n+1} \prod_{l=1}^{n} \frac{\lambda_{j} \lambda_{l}-\lambda_{j}+1}{\lambda_{j} \lambda_{l}-\lambda_{l}+1} \quad j=1, \cdots$, , 44.63$)$

Se fizermos as seguintes parametrizações

$$
\begin{aligned}
& \lambda_{j}=\frac{\sinh \left(i \lambda_{0}-\sigma_{j}\right)}{\sinh \left(\sigma_{j}\right)}, \\
& \frac{b^{2}}{c^{2}-a_{1}-b^{2}}=\frac{\sin (\sigma)}{\sin (\lambda-\sigma)}, \\
& \frac{b c}{c^{2}-a_{1}-b^{2}}=\frac{\sin (\lambda)}{\sin (\lambda-\sigma)}, \quad \sigma \neq \lambda, \quad \lambda_{0}=\frac{2 \pi}{3},
\end{aligned}
$$

a equação (4.63) é reescrita como

$$
\begin{aligned}
& \left(\frac{\sin \left(\lambda_{0}+i \sigma_{j}\right)}{\sin \left(i \sigma_{j}\right)}\right)^{N}\left(\frac{\sinh \left(i \lambda_{0}+\sigma_{j}\right) \sin (\sigma-\lambda)-\sin (\sigma) \sinh \left(\sigma_{j}\right)}{\sinh \left(i \lambda_{0}+\sigma_{j}\right) \sin (\sigma)-\sin (\sigma+\lambda) \sinh \left(\sigma_{j}\right)}\right)^{N}= \\
& -\prod_{l=1}^{n} \frac{\sinh \left(\sigma_{j}-\sigma_{l}-i \lambda_{0}\right)}{\sinh \left(\sigma_{j}-\sigma_{l}+i \lambda_{0}\right)}, \quad j=1 \ldots, n,
\end{aligned}
$$

Repare que quando $\lambda=\lambda_{0}$ obtemos

$$
\begin{aligned}
& {\left[\frac{\sinh \left(i \lambda_{0}-\sigma_{j}\right) \sinh \left(i \lambda_{0}-i \sigma-\sigma_{j}\right)}{\sinh \left(\sigma_{j}\right) \sinh \left(i \lambda_{0}+\sigma_{j}\right)}\right]^{N}=} \\
& -\prod_{l=1}^{n} \frac{\sinh \left(\sigma_{j}-\sigma_{l}-i \lambda_{0}\right)}{\sinh \left(\sigma_{j}-\sigma_{l}+i \lambda_{0}\right)}, \quad j=1, \ldots, n,
\end{aligned}
$$

que coincide com a equação do modelo de 6 vértices com anisotropia $\Delta=-\cos \left(\lambda_{0}\right)=$ $\frac{1}{2}$. 
Este último resultado é bastante interessante e nos diz sobre a existência de um novo modelo de 10 vértices, que pode ser pensado como uma "deformação" do modelo de 6 vértices usual em $\Delta=\frac{1}{2}$. O modelo de 6 vértices com anisotropia $\Delta=\frac{1}{2}$ possui propriedades bastantes curiosas, sendo a sua função de partição relacionada ao clássico problema estatístico de contagem de matrizes com sinais alternados, conexão esta que tem motivado um grande número de trabalhos (veja por exemplo [25]- [28]). Uma deformação parecida à que encontramos neste trabalho foi encontrada em [29] no estudo de Hamiltonianos quânticos de spin 1 (relacionados a modelo de 19 vértices). Foi também encontrado um modelo integrável com equação do ansatz de Bethe que pode ser interpretada como uma deformações da cadeia $X X Z$ (relacionada com o modelo de 6 vértices) com anisotropia $\Delta=\frac{1}{2}$. A formulação da matriz de transferência linha-para-linha dos modelos de 10 vértices, indicam que tais modelos são relacionados com cadeias quânticas de spin misto, onde os sítios pares (ímpares) são ocupados por matrizes $S U(2)$ de spin $\frac{1}{2}(1)$. Nosso resultado indica a existência de uma nova cadeia quântica de spin misto, que poderá também ser interpretada, assim como em [29], como uma deformação de cadeia $X X Z$ com anisotropia $\Delta=\frac{1}{2}$. O estudo das propriedades físicas do nosso modelo de vértices bem como a possível obtenção da cadeia quântica de spins mistos associada são sem dúvida interessantes trabalhos a serem feitos futuramente. 


\section{Capítulo 5}

\section{Conclusão}

Nesta dissertação fizemos as primeiras aplicações do ansatz do produto matricial [8] em modelos de vértices associados a matrizes de transferência. Este novo ansatz oferece a princípio algumas vantagens em relação ao ansatz de Bethe na sua versão de coordenadas. A primeira delas é a forma minemômica de como associamos os sítios ocupados e vazios da rede com as matrizes que compõe o ansatz e que permitem simples generalizações para situações gerais. Uma outra vantagem provém da simplicidade da obtenção das equações que fixam o espectro do operador a ser diagonalizado em questão (provém da propriedade cíclica do produto matricial).

Estudamos duas famílias de modelos de vértices.

$\mathrm{Na}$ primeira delas introduzimos novos modelos de vértices, que denominamos por modelos de 5 vértices interagentes. Nestes modelos os vértices possuem além das interações usuais de vizinhos próximos dadas pela regra do gelo, interações ao longo da diagonal, sendo as mesmas de natureza repulsiva e caracterizada por um parâmetro $s$. A matriz de transferência diagonal-para-diagonal de tais modelos pode ser vizualizada como um operador que governa as flutuações de um modelo de vértices em que as flechas possuem um "tamanho" efetivo $s$. Quando todas as flechas possuem tamanho $s=1$, recuperamos o tradicional modelos de 6 vértices formulado na matriz de transferência diagonal-para-diagonal, e resolvido por Truong e Schotte [18] 
mediante o uso do tradicional ansatz de Bethe de coordenadas.

Como inexistem na literatura estudos analíticos ou numéricos do espectro de matrizes de transferência diagonal-para-diagonal, fizemos tal estudo para o caso do modelo de 6 vértices. Analizando-se as equações que fixam o espectro do modelo (análogas às equações do ansatz de Bethe) obtidas para o caso da matriz de transferência diagonal-para-diagonal, verificamos, como esperado, que a anergia livre no limite termodinâmico converge para os mesmos valores calculados usando-se a matriz de transferência linha-para-linha [3]. É importante mencionarmos que as equações que fixam o espectro da matriz de transferência linha-para-linha são mais simples, quando comparadas com as equações associadas a matriz de transferência diagonal-paradiagonal, ou seja, se por um lado determinamos com mais facilidade a forma e as relações que fixam o espectro do modelo, por outro tais relações tornam-se mais complicadas que as obtidas no caso da matriz de transferência linha-para-linha.

Na segunda classe de modelos estudados abordamos os chamados modelos de 10 vértices. Através do ansatz de produto matricial conseguimos obter as condições que garantem as integrabilidade do modelo. Encontramos três ramos de soluções não triviais. No primeiro ramo, indentificamos os modelos de 6 vértices. No segundo ramo, encontramos um genuíno modelo de 10 vértices. Um modelo nesta classe foi encontrado por de Vega e Woynarovich [20] usando o método da fusão associado à matriz de transferência linha-para-linha. Entretanto a solução apresentada por eles, é um caso particular da solução encontrada por nós. No terceiro ramo, encontramos um novo modelo de 10 vértices. Este modelo pode ser visto como uma deformação do modelo de 6 vértices valores da com anisotropia $\Delta=\frac{1}{2}$. Este resultado indica a existência de uma nova cadeia quântica de spin misto (spin $\frac{1}{2}$ e spin 1) ainda desconhecida na literatura. Como o modelo de 6 vértices está intimamente ligado com a Hamiltoniana $X X Z$, tal Hamiltoniana poderá ser interpretada como uma deformação da cadeia quântica $X X Z$ com anisotropia $\Delta=\frac{1}{2}$. O estudo das propriedades críticas das soluções gerais do modelo de 10 vértices do segundo ramo, bem como a derivação da cadeia quântica associada aos modelos de vértices do segundo ramo são 
interessantes continuações das pesquisas aqui apresentadas. 


\section{Capítulo 6}

\section{Bibliografia}

[1] - C. N. Yang and C. P. Yang, Phys. Rev. 150, 321 (1966).

[2] - E. H. Lieb, Phys. Rev. 162, 162 (1967);

[3] - R. J. Baxter, Exactly Solved Models in Statistical Mechanics (Academic Press: New York) (1982).

[4] - R.B. Stinchombe, and G.M. Schütz, Phys. Rev. Lett. 75, 140 (1995);Europhys Lett. 29, 663 (1995)

[5] - T. Sasamoto, and M. Wadati, J. Phys. Soc. Japan 66, 2618 (1999).

[6] - V. Popkov, M.E. Fouladvand and G.M Schütz, J. Phys. A. 35, 8187 (2002).

[7] - V. Popkov and G.M. Schütz, Math. Phys. Anal. Geom 9, 401 (2002).

[8] - F. C. Alcaraz and M. J. Lazzo, J. Phys. A 37, L1-L7 (2004).

[9] - K. G. Wilson and J. Kogut, Phys. Reports 12C, 75-199 (1974).

[10] - A.A. Belavin, A.M. Polyakov and A.B. Zamolodchikov, Nucl. Phys. B241, 333 (1984).

[11] - D. Friedan, Z. Qiu and S. Shenker Alcaraz Phys. Lett. 151B, 37 (1985).

[12] - Kac V. G. Group Theor. Meth. in Phys., (Springer Verlag: Berlin) (1979). 
[13] - H.W.J. Blote, J.L. Cardy, and M.P. Nightingale, Phys. Rev. Lett. 56, 742 (1986).

[14] - I.Affleck, Phys. Rev. Lett. 56, 746 (1986).

[15] - J. L. Cardy, Scaling and Renormalization in Statistical Physics, (Cambrige University Press:Cambrige) (1996).

[16] - J.L. Cardy, Nucl. Phys. B270 , 186 (1986).

[17] - R. Z. Bariev, Mat. Fiz. 49, 261 (1981).

[18] - T. T. Truong and K. D. Schotte, Nucl. Phys. B20[FS8], 77-101 (1983).

[19] - F.C. Alcaraz, M. N. Barber and M. T. Batchelor, Ann. Phys. 182, 280-97 (1998).

[20] - H. J. de Vega and F. Woynarovich, J. Phys. A: Math. Gen. 25, 4499 (1992).

[21] - J.B Kogut, Rev. Mod. Phys. 51, 659 (1979).

[22] - M. Henkel, Conformal Invariance and Critical Phenomena, (Springer Verlag: Berlin) (1999).

[23] - J.L. Cardy, Nucl. Phys. B275 [FS17], 200 (1986).

[24] - L.P. Kadanoff and A.C. Brown, Annals of Phys. 121, 318-342 (1979).

[25] - Razumov A.V and Stroganov Yu.G. J. Phys. A 34, L179 (2001).

[26] - Batchelor M.T. de Gier J. and Nienhuis B. J. Phys. A 34, L265 (2001).

[27] - Razumov A.V and Stroganov Yu.G. J. Phys. A 34, 5335 (2001).

[28] - Pearce P.A., Rittenberg V. and de Gier J., Preprint. arXiv cond-mat/0108051. (2001).

[29] - de Gier, Batchelor M.T., Nienhuis B. and Mitras S.Pearce, Preprint. arXiv cond-mat/0110011. (2001).

[30] - B. Derrida, M. R. Evans, V. Hakim and D. Mukamel, J. Phys. A 26, 143 (1993). 
[31] - B. Derrida, E. Domany and D. Mukamel, J. Stat. Phys. 69, 667 (1992).

[32] - S. Wolfram, Mathematica: a system for doing mathematics by computer 2 ed., (Addison-Wesley: Redwood City) (1991).

[33] - Alcaraz F.C. and Bariev R.Z., Phys. A 306, 51 (2002). 